\title{
Novel 2,6-diketopiperazine-derived acetohydroxamic acids as promising anti-Trypanosoma brucei agents
}

\author{
George Fytas*, 1 , Grigoris Zoidis**, 1, Martin C Taylor², John M Kelly², Alexandra \\ Tsatsaroni $^{1} \&$ Andrew Tsotinis ${ }^{1}$ \\ ${ }^{1}$ Department of Pharmaceutical Chemistry, Faculty of Pharmacy, School of Health Sciences, National \& \\ Kapodistrian University of Athens, Panepistimiopolis-Zografou, GR-15771 Athens, Greece \\ ${ }^{2}$ Department of Pathogen Molecular Biology, London School of Hygiene \& Tropical Medicine, Keppel Street, \\ London WC1E 7HT, UK \\ *Author for correspondence: Tel.: +30 210727 4810; Fax: +30 210727 4747; gfytas@pharm.uoa.gr \\ **Author for correspondence: Tel.: +30 210727 4809; Fax: +30 210727 4747; zoidis@pharm.uoa.gr
}

- Aim: Identification of new effective and selective trypanocidal agents. Materials \& methods: Twelve novel acetohydroxamic acid derivatives based on 2-alkyl-2-aryl-2,6-diketopiperazine scaffolds have been synthesized and evaluated in vitro for their growth inhibitory activity against bloodstream form $T$. brucei. Results: All the analogues were remarkably potent inhibitors, with low micromolar to submicromolar activities. Structureactivity relationship studies demonstrated that the presence of an alkyl substituent at the $N(4)$-position of the 2,6-diketopiperazine ring portion was, in general, beneficial to trypanocidal activity in this series. Conclusions: The highest activity resulted from the introduction of a methyl, $n$-propyl or $n$-butyl substituent to the $N(4)$ position of the parent compound. Importantly, the most potent analogues were found to be highly selective against $T$. brucei with respect to mammalian cells.

- Graphical abstract: optional - if the authors wish, they can include a Figure to appear that is representative of their article.
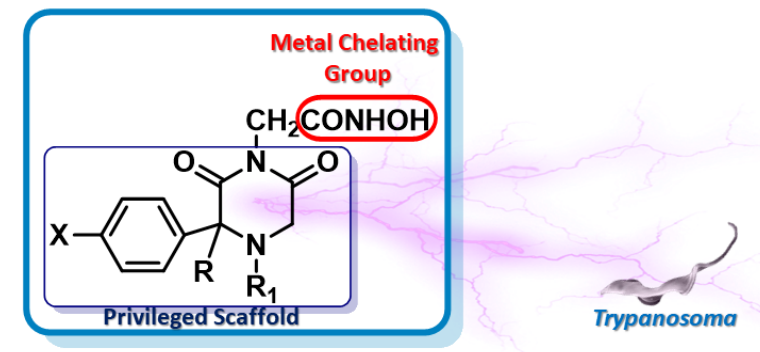

R: $\mathrm{CH}_{3},\left(\mathrm{CH}_{2}\right)_{2} \mathrm{CH}_{3},\left(\mathrm{CH}_{2}\right)_{3} \mathrm{CH}_{3}$

$\mathbf{R}_{1}: \mathrm{H}, \mathrm{CH}_{3},\left(\mathrm{CH}_{2}\right)_{2} \mathrm{CH}_{3},\left(\mathrm{CH}_{2}\right)_{3} \mathrm{CH}_{3}$ X: $\mathrm{H}, \mathrm{F}, \mathrm{NO}_{2}$

- Keywords: 2-alkyl-2-aryl-2,6-diketopiperazine-1-acetohydroxamic acids, Anti-trypanosomal activity, Cytotoxicity on mammalian cells, NMR.

\section{- Main body of text:}




\section{Introduction}

Human African Trypanosomiasis (HAT) or sleeping sickness is amongst the most serious neglected tropical diseases and is caused by infection with parasitic protozoa of the Trypanosoma brucei subspp [1,2]. HAT constitutes a major public health risk within 36 sub-Saharan Africa countries due to its epidemic character $[2,3]$. It is estimated that 70 million people are at risk, and that around 3000 new infections occur every year in the endemic disease foci [2]. Current treatments for HAT have been based on old drugs including, suramin, pentamidine, melarsoprol and eflornithine, with an eflornithine-nifurtimox combination introduced in 2009 $[1,2,4]$ although oral fexinidazole has shown considerable promise in clinical trial and has recently been recommended for use ([5] Ku Mesu, V.K.B.K., Kalonji, W.M., Bardonneau, C., Mordt, O.V., Blesson, S. (2018) Oral fexinidazole for late-stage African Trypanosoma brucei gambiense trypanosomiasis: a pivotal multicentre, randomised, non-inferiority trial. Lancet 391, 144-154. [6] European Medicines Agency recommends fexinidazole, the first all-oral treatment for sleeping sickness. https://www.dndi.org/2018/media-centre/pressreleases/). These drugs are often associated with severe toxic side effects, poor efficacy, and problematic administration. Additionally, the HAT drugs are expensive, and their usage requires adequate medical care, which is not readily available in the most affected regions of sub-Saharan Africa [2,4]. All the above issues with the existing HAT drugs emphasize an imperative need for research and development of new efficient, safe, and affordable antitrypanosomal therapeutics.

In 1999, it was discovered that bloodstream form $T$. brucei are sensitive in vitro to the anti-influenza A drugs amantadine I and rimantadine II. Rimantadine was also found to be toxic to the trypanosomatid parasites $T$. cruzi and Leishmania major. ${ }^{5}$ Two years later, it was reported that a series of aminoadamantane and aminoalkylcyclohexane derivatives are effective growth inhibitors of $T$. brucei in vitro and in vivo, and that inhibition was correlated with the hydrophobicity of the compounds. Some of these derivatives (III-IV, Figure 1) showed submicromolar trypanocidal activities in vitro; in particular the adamantane analogue III $\left(\mathrm{IC}_{50}=0.33 \mu \mathrm{M}\right)$ gave 400- and 21-fold increases in antitrypanosome potency compared to amantadine and rimantadine, respectively. ${ }^{6}$ In our earlier works we communicated the trypanocidal properties of some nitrogen-containing adamantane derivatives (amines or not). ${ }^{7,8}$ Among them, compounds V-VII (Figure 1) possessed considerable activities in vitro against $T$. brucei. Oxazolone $\mathbf{V}^{8}$ was the most active inhibitor of the parasite growth, exhibiting a potency that was 3 -fold higher than rimantadine and at least 45 -fold greater than amantadine, while the trypanocidal activity of spiro piperidine $\mathbf{V I}^{7}$ was found to be 1.5 times more than rimantadine, and at least 25 times greater than amantadine. The spiro barbituric analogue $\mathrm{VII}^{7}$ displayed more potent inhibition (7-fold) than that of amantadine, although it was 3 -fold less effective than rimantadine.

In pursuit of a better antitrypanosome potency we explored the trypanocidal properties of the structurally related spiro 2,6-diketopiperazine derivatives VIIIa and VIIIb. Unfortunately, these compounds were only marginally active against $T$. brucei parasite. Yet, compounds VIIla and VIIIb as well as other lipophilic spiro carbocyclic 2,6-DKPs represent useful scaffolds that can be transformed into potent trypanocidal agents, with single nanomolar to submicromolar activities, by introducing an acetohydroxamic acid moiety to their imidic nitrogen [5,6]. Thus, we produced a series of lipophilic, constrained spiro carbocyclic 2,6-diketopiperazine-1acetohydroxamic acid derivatives (Fig. 1., 1a-g, 2, 3, 4a-d, 4f, 5a, 5b, 5d) that displayed single nanomolar to submicromolar activities. [5,6] SAR studies showed the indispensability of the hydroxamic unit (CONHOH) for the trypanocidal activity in this class of compound [5]. Thus, we presumed that these hydroxamates act by inhibiting a decisive parasite metalloenzyme due to the metal ion coordinating properties exerted by the hydroxamic acid group in the catalytic site. We have also confirmed that incorporating a benzyl rather than an aliphatic substituent into the methylene carbon next to the basic nitrogen of the spiro carbocyclic 2,6-DKP portion leads to analogues (Fig. 1., 1f, 1g, 2, $\mathbf{3}$ and $\mathbf{4 f}$ ) with the higher trypanocidal activity. 
In order to identify the structural features of the 2,6-DKP-based acetohydroxamic acids required for potent trypanocidal activity, we modified the spiro carbocyclic 2,6-DKP core structure by changing the spiro-linked carbocycle component for an alkyl and an aryl substituent. In this report, we present the design and synthesis of a new series of acetohydroxamic acid analogues (Fig. 1. 6-17) as T. brucei growth inhibitors based on conformationally non-constrained 3-alkyl-3-aryl-2,6-DKP scaffolds. Within this series, we studied the trypanocidal potency of compounds in relation to: (a) the length of the C-3 $n$-alkyl substituent (compounds 68), (b) the para-substitution on the aromatic ring with fluorine atom or nitro group (compounds 9 and 10), and (c) the alkyl substitution at the $N(4)$-position of the 2,6-DKP ring (compounds 11-17). The antitrypanosomal properties of these novel compounds were assessed against $T$. brucei bloodstream form parasites in vitro.

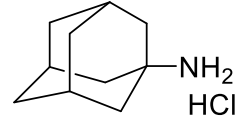

Amantadine I

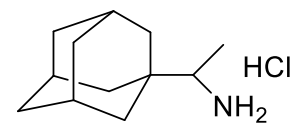

Rimantadine II

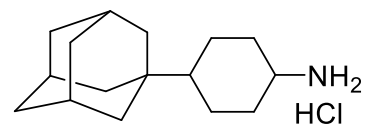

III: $\mathrm{IC}_{50}=0.33 \mu \mathrm{M}$

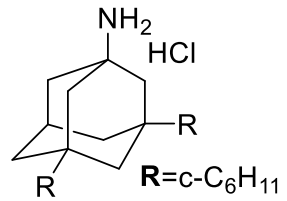

IV: $I_{50}=0.37 \mu \mathrm{M}$<smiles>O=C1ON=C2C3CC4CC(C3)CC12C4</smiles>

V: $I C_{50}=2.87 \mu \mathrm{M}$

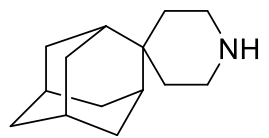

VI: $I_{50}=5.28 \mu \mathrm{M}$<smiles>O=C1NC(=O)C23CC4CC(CC(C4)C2C1=O)C3</smiles>

VII: $\mathrm{IC}_{50}=20.0 \mu \mathrm{M}$<smiles>[R]C1C(=O)NC(=O)C2(C3CC4CC(C3)CC2C4)N1[R]</smiles>

VIII a, b<smiles>[R]C1C(=O)N(CC(=O)NO)C(=O)C2(C3CC4CC(C3)CC2C4)N1[R]</smiles>

1a-g, 2, 3<smiles>[R]C1C(=O)N(CC(=O)NO)C(=O)C2(CCCCC2)C1[R]</smiles>

$\mathrm{n}=7:$ : $a-d, 4 f$ $n=6: 5 a, 5 b, 5 d$

a: $\mathrm{R}=\mathrm{R}_{1}=\mathrm{H}$

b: $\mathrm{R}=\mathrm{H}, \mathrm{R}_{1}=\mathrm{CH}_{3}$

c: $\mathrm{R}=\mathrm{CH}_{3}, \mathrm{R}_{1}=\mathrm{H},(\mathrm{S})$-enantiomer

d: $\mathrm{R}=\mathrm{CH}_{2} \mathrm{CH}\left(\mathrm{CH}_{3}\right)_{2}, \mathrm{R}=\mathrm{H},(\mathrm{S})$-enantiomer

e: $\mathrm{R}=\mathrm{CH}_{2} \mathrm{CH}_{2} \mathrm{SCH}_{3}, \mathrm{R}$ 仿, (S)-enantiomer

f: $\mathrm{R}=\mathrm{CH}_{2} \mathrm{C}_{6} \mathrm{H}_{5}, \mathrm{R} \mathrm{R}_{1}=\mathrm{H},(\mathrm{S})$-enantiomer

g: $\mathrm{R}=\mathrm{CH}_{2} \mathrm{C}_{6} \mathrm{H}_{5}, \mathrm{R}_{1}=\mathrm{H},(R)$-enantiomer

2: $\mathrm{R}=\mathrm{CH}_{2} \mathrm{C}_{6} \mathrm{H}_{5}, \mathrm{R}_{1}=\mathrm{H}$, racemic

3: $\mathrm{R}=\mathrm{CH}_{2} \mathrm{C}_{6} \mathrm{H}_{4} \mathrm{Cl}-4, \mathrm{R}_{1}=\mathrm{H}$, racemic<smiles>[X]c1ccc(C2([R])C(=O)N(CC(N)=O)C(=O)CN2[R])cc1</smiles>

6-17

6: $\mathrm{R}=\mathrm{CH}_{3}, \mathrm{R}_{1}=\mathrm{X}=\mathrm{H}$
7: $\mathrm{R}=\left(\mathrm{CH}_{2}\right)_{2} \mathrm{CH}_{3}, \mathrm{R}_{1}=\mathrm{X}=\mathrm{H}$
8: $\mathrm{R}=\left(\mathrm{CH}_{2}\right)_{3} \mathrm{CH}_{3}, \mathrm{R}_{1}=\mathrm{X}=\mathrm{H}$
9: $\mathrm{R}=\mathrm{CH}_{3}, \mathrm{R}_{1}=\mathrm{H}, \mathrm{X}=\mathrm{F}$
10: $\mathrm{R}=\mathrm{CH}_{3}, \mathrm{R}_{1}=\mathrm{H}, \mathrm{X}=\mathrm{NO}_{2}$
11: $\mathrm{R}=\mathrm{R}_{1}=\mathrm{CH}_{3}, \mathrm{X}=\mathrm{H}$
12: $\mathrm{R}=\left(\mathrm{CH}_{2}\right)_{2} \mathrm{CH}_{3}, \mathrm{R}_{1}=\mathrm{CH}_{3}, \mathrm{X}=\mathrm{H}$
13: $\mathrm{R}=\left(\mathrm{CH}_{2}\right)_{3} \mathrm{CH}_{3}, \mathrm{R}_{1}=\mathrm{CH}_{3}, \mathrm{X}=\mathrm{H}$
14: $\mathrm{R}=\mathrm{R}_{1}=\mathrm{CH}_{3}, \mathrm{X}=\mathrm{F}$
15: $\mathrm{R}=\mathrm{R}_{1}^{\prime}=\mathrm{CH}_{3}, \mathrm{X}=\mathrm{NO}_{2}$
16: $\mathrm{R}=\mathrm{CH}_{3}, \mathrm{R}_{1}=\left(\mathrm{CH}_{2}\right)_{2} \mathrm{CH}_{3}, \mathrm{X}=\mathrm{H}$
17: $\mathrm{R}=\mathrm{CH}_{3}, \mathrm{R}_{1}=\left(\mathrm{CH}_{2}\right)_{3} \mathrm{CH}_{3}, \mathrm{X}=\mathrm{H}$

Figure 1. Structures of amantadine I, rimantadine II, lipophilic adamantane derivatives III-VII, 2,6-DKPs VIIIa and VIIIb, spiro carbocyclic 2,6-diketopiperazine-1-acetohydroxamic acid derivatives 1a-g, 2, 3, 4a-d, 4f, 5a, 5b, 5d reported previously $[\mathrm{X}-\mathrm{X}]$, and structures of the new acetohydroxamic acid analogues 6-17 based on 3-alkyl-3aryl-2,6-diketopiperazine scaffolds.

\section{Results and Discussion}

\subsection{Chemistry}


Compounds 6-17 were synthesized following similar procedures to those reported in our previous publications (Schemes 1 and 2) [5-7]. As shown in Scheme 1, the Strecker reaction of the ketones 18-22 with ethyl glycinate hydrochloride and sodium cyanide, and subsequent acidic hydration $\left(\mathrm{H}_{2} \mathrm{SO}_{4} 97 \%\right)$ of the unstable $\alpha$-aminonitrile intermediates (not shown) provided the respective amide-ester derivatives 23-27, which served as key compounds for further elaboration. Treatment of compounds 23-27 with potassium bis(trimethylsilyl)amide in THF gave, after an SN2 reaction of the intermediate potassium imidate salts with benzyl or 4-methoxybenzyl bromoacetate in DMF, the corresponding 2,6-DKP-1-acetic acid benzyl ester derivatives 28-32. An analogous base-catalyzed intramolecular cyclization of the amide-ester derivatives 23-27 using potassium bis(trimethylsilyl)amide (1eq), followed by the addition of TFA (1eq) led to their respective 2,6-DKPs 42-46. Reductive methylation on the basic nitrogen atom of the 2,6-DKPs $42-46$ with $\mathrm{CH}_{2} \mathrm{O} / \mathrm{NaCNBH}_{3}$ in $\mathrm{MeOH}$ or $\mathrm{MeOH}-\mathrm{THF}$ 1:1 gave the corresponding methyl substituted analogues 47-51. The latter compounds, upon reaction with benzyl or 4-methoxybenzyl bromoacetate in the presence of sodium hydride in DMF, were converted to the N-methylated 2,6-DKP-1-acetic acid benzylester derivatives 52-56. Catalytic hydrogenolysis $\left(\mathrm{H}_{2} / 10 \% \mathrm{Pd}-\mathrm{C}\right)$ of the benzyl esters 28-31 and 52-55 occurred cleanly to afford the carboxylic acids 33-36 and 57-60, which underwent efficient $C D I$ coupling reactions with $O$-benzylhydroxylamine to give the $O$-benzyl hydroxamates 37-40 and 61-64, respectively. The desired hydroxamic acids 6-9 and 11-14 were available via catalytic hydrogenolysis $\left(\mathrm{H}_{2} / 10 \% \mathrm{Pd}-\mathrm{C}\right)$ of the benzyl-protected hydroxamates 37-40 and 61-64, respectively. Additionally, treatment of the 4-methoxybenzyl esters $\mathbf{3 2}$ and $\mathbf{5 6}$ with TFA, followed by CDI-catalyzed coupling reactions of the respective carboxylic acid intermediates (not shown) with 0 -(4-methoxybenzyl)hydroxylamine gave the corresponding $O$-(4-methoxybenzyl) hydroxamates $\mathbf{4 1}$ and $\mathbf{6 5}$. The removal of the 4-methoxybenzyl protecting group of $\mathbf{4 1}$ and $\mathbf{6 5}$ was achieved by exposure to TFA in the presence of triethylsilane in $\mathrm{CH}_{2} \mathrm{Cl}_{2}$ affording the targeted nitro-substituted hydroxamic acid analogues $\mathbf{1 0}$ and $\mathbf{1 5}$, respectively. 


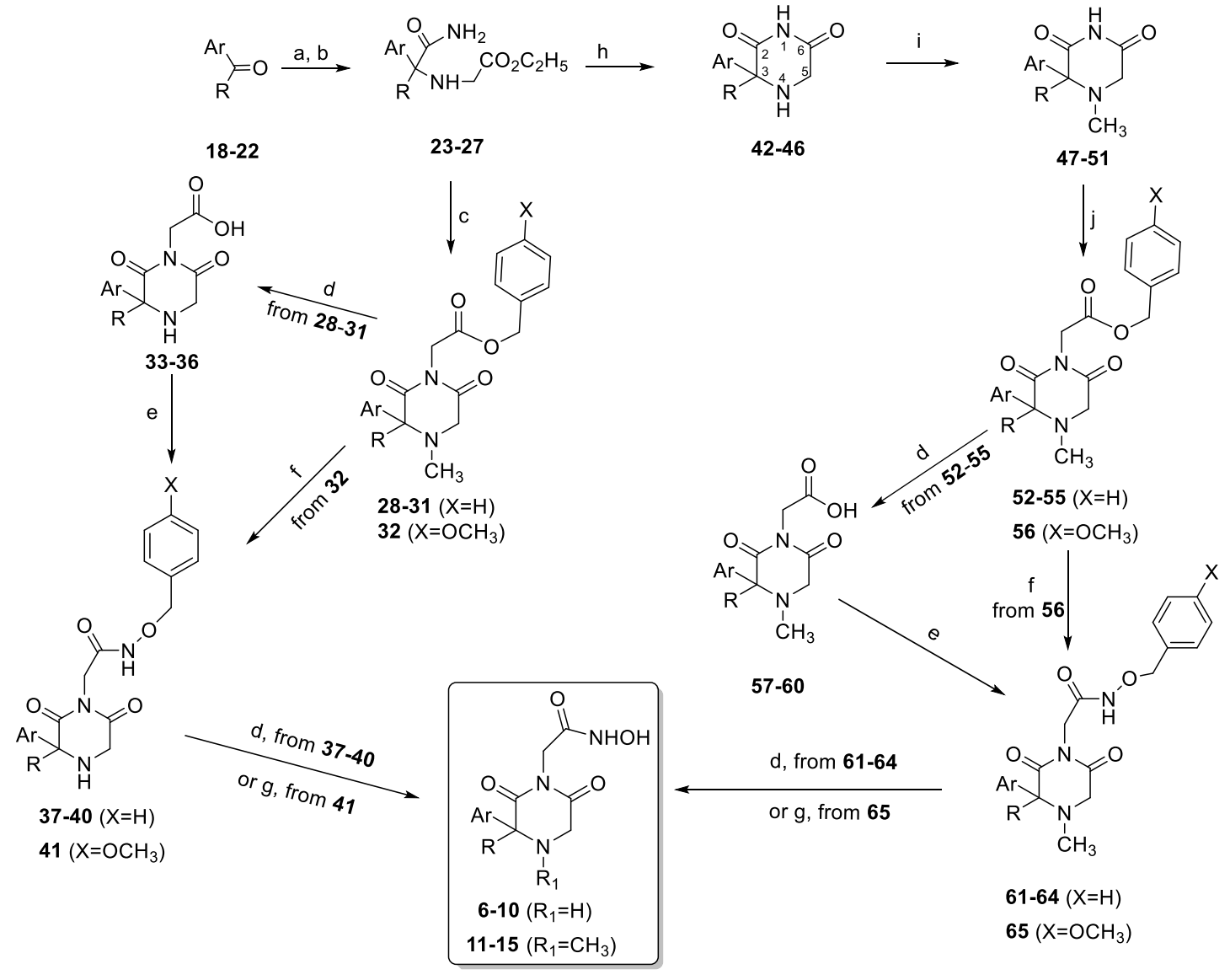

$18,23,28,33,37,6,42,47,52,57,61,11: \mathrm{R}=\mathrm{CH}_{3}, \mathrm{Ar}=\mathrm{C}_{6} \mathrm{H}_{5}$

19, 24, 29, 34, 38, 7, 43, 48, 53, 58, 62, 12: $\mathrm{R}=\left(\mathrm{CH}_{2}\right)_{2} \mathrm{CH}_{3}, \mathrm{Ar}=\mathrm{C}_{6} \mathrm{H}_{5}$

20, 25, 30, 35, 39, 8, 44, 49, 54, 59, 63, 13: $\mathrm{R}=\left(\mathrm{CH}_{2}\right)_{3} \mathrm{CH}_{3}, \mathrm{Ar}=\mathrm{C}_{6} \mathrm{H}_{5}$

21, 26, 31, 36, 40, 9, 45, 50, 55, 60, 64, 14: $\mathrm{R}=\mathrm{CH}_{3}, \mathrm{Ar}=4-\mathrm{FC}_{6} \mathrm{H}_{4}$

$22,27,32, \quad 41,10,46,51,56, \quad 65,15: \mathrm{R}=\mathrm{CH}_{3}, \mathrm{Ar}=4-\mathrm{NO}_{2} \mathrm{C}_{6} \mathrm{H}_{4}$

Scheme 1. Reagents and conditions: (a) $\mathrm{NaCN}, \mathrm{H}_{2} \mathrm{NCH}_{2} \mathrm{CO}_{2} \mathrm{Et} . \mathrm{HCl}, \mathrm{DMSO} / \mathrm{H}_{2} \mathrm{O}$ 29:1 (v/v), rt, 48h; (b) (i) $\mathrm{H}_{2} \mathrm{SO}_{4}$ $97 \%, \mathrm{CH}_{2} \mathrm{Cl}_{2}$, rt, 24h; (ii) ice and then aq. $\mathrm{NH}_{3} 26 \%$ to $\mathrm{pH} 7-8,20-55 \%$ yields over two steps; (c) (i) ( $\left.\mathrm{Me}_{3} \mathrm{Si}\right)_{2} \mathrm{NK}$ (1 eq), THF, 0-5 ${ }^{\circ} \mathrm{C}$ then rt, 2h, argon; (ii) $\mathrm{BrCH}_{2} \mathrm{CO}_{2} \mathrm{CH}_{2} \mathrm{C}_{6} \mathrm{H}_{5}$ or $\mathrm{BrCH}_{2} \mathrm{CO}_{2} \mathrm{CH}_{2} \mathrm{C}_{6} \mathrm{H}_{5} \mathrm{OCH}_{3}-4$ only for 32, DMF, rt, 48h, argon, 73-91\%; (d) $\mathrm{H}_{2} / \mathrm{Pd}-\mathrm{C}$, EtOH or EtOH-AcOEt 3:1 for 35, 50 psi, rt, 3h, 96- >99\% for 33-36, 57-60, 75-94\% for 6-9, 11-14; (e) (i) CDI, THF for 37-40, 61-63 or THF-DMF 4:1 for $64,28^{\circ} \mathrm{C}$ for 37-39, 61-63 or $55^{\circ} \mathrm{C}$ for $40,64,1 \mathrm{~h}$, argon; (ii) $\mathrm{C}_{6} \mathrm{H}_{5} \mathrm{CH}_{2} \mathrm{ONH}_{2} . \mathrm{HCl}, \mathrm{Et}_{3} \mathrm{~N}, 28^{\circ} \mathrm{C}$, 24h and then $45^{\circ} \mathrm{C}$, $1 \mathrm{~h}$, argon, for 37-39, 61-63 or $55{ }^{\circ} \mathrm{C}, 25 \mathrm{~h}$, argon, for 40, 64, 61-76\%; (f) (i) $\mathrm{CF}_{3} \mathrm{CO}_{2} \mathrm{H}, \mathrm{CH}_{2} \mathrm{Cl}_{2}, \mathrm{rt}, 90 \mathrm{~min}$; (ii) $\mathrm{Et}_{3} \mathrm{~N}, \mathrm{CDI}, \mathrm{THF}, 28{ }^{\circ} \mathrm{C}, 1 \mathrm{~h}$, argon; (iii) 4$\mathrm{CH}_{3} \mathrm{OC}_{6} \mathrm{H}_{4} \mathrm{CH}_{2} \mathrm{ONH}_{2}, 28{ }^{\circ} \mathrm{C}$, $18 \mathrm{~h}$, then $55^{\circ} \mathrm{C}$, $7 \mathrm{~h}$, argon, $48 \%$ and $43 \%$ yields over two steps for $\mathbf{4 1}$ (from 32 ) and 65 (from 56), respectively; (g) $\mathrm{CF}_{3} \mathrm{CO}_{2} \mathrm{H}, \mathrm{CH}_{2} \mathrm{Cl}_{2}, \mathrm{rt}, 10 \mathrm{~min}$, then $\mathrm{Et}_{3} \mathrm{SiH}$, rt, $45 \mathrm{~min}, 63 \%$ for 10 (as hydrochloride) from 41, 70\% for 15 from 65; (h) as (c) (i), then $\mathrm{CF}_{3} \mathrm{CO}_{2} \mathrm{H}$ (1 eq) 91-96\%; (i)(i) aq $\mathrm{CH}_{2} \mathrm{O} 37 \%$, $\mathrm{MeOH}$ or $\mathrm{MeOH}-\mathrm{THF}$ 1:1 for 51, rt, 3h, then $\mathrm{NaCNBH}_{3}$, rt, $4 \mathrm{~h}$ at pH 6-7 (maintaining by adding $\mathrm{AcOH}$ ); (ii) $\mathrm{NaOH} 1 \mathrm{~N}$ and $\mathrm{Na}_{2} \mathrm{CO}_{3}$ to pH 8, 74-88\%; (j) $\mathrm{NaH}, \mathrm{DMF}$, rt, $1 \mathrm{~h}$ or 10 min for 56, argon and then as (c) (ii) using $\mathrm{BrCH}_{2} \mathrm{CO}_{2} \mathrm{CH}_{2} \mathrm{C}_{6} \mathrm{H}_{5}$ or $\mathrm{BrCH}_{2} \mathrm{CO}_{2} \mathrm{CH}_{2} \mathrm{C}_{6} \mathrm{H}_{5} \mathrm{OCH}_{3}-4$ only for $56,71-87 \%$.

Scheme 2 shows the synthesis of the hydroxamic acid analogues 16 and 17, bearing a $n$-propyl (16) or $n$-butyl (17) aliphatic substituent at the basic nitrogen atom of the 2,6-DKP scaffold. Treatment of acetophenone 18 with $n$-propylamine or $n$-butylamine hydrochloride and sodium cyanide, followed by acid-catalyzed hydration of the unstable $\alpha$-aminonitrile intermediates (not shown) gave the respective amino amides $\mathbf{6 6}$ and $\mathbf{6 7}$. These 
compounds were then reacted with ethyl bromoacetate in the presence of sodium bicarbonate in DMF to provide the corresponding amide-ester derivatives $\mathbf{6 8}$ and $\mathbf{6 9}$. Employing a four step reaction sequence similar to that described above for the preparation of the hydroxamic acid congeners 6-9, the amide-ester derivatives 68 and 69 were converted to the hydroxamic acid analogues 16 and 17 , respectively. The ${ }^{1} \mathrm{H}$ and ${ }^{13} \mathrm{C} \mathrm{NMR}$ spectra for the acetohydroxamic acid analogues described in this report (compounds 6-17) are consistent with E/Z conformational behavior of these molecules in solution. The assignment of the $E$ and $Z$ isomers was based on our E/Z conformational isomerism study reported previously [8].

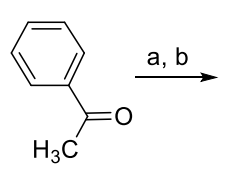

18

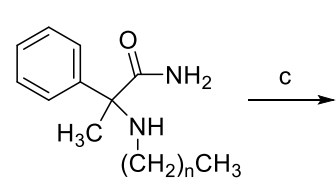

66: $n=2$ 67: $n=3$

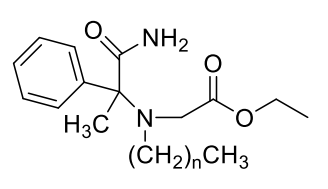

68: $n=2$

69: $n=3$

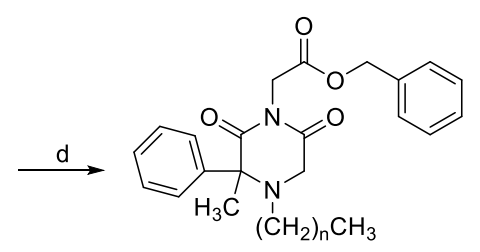

70: $n=2$

71: $n=3$

$\longrightarrow$

72: $n=2$

73: $n=3$
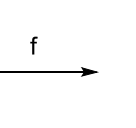

74: $n=2$

75: $n=3$

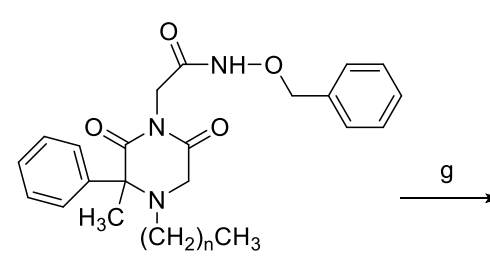

$\mathrm{g}$

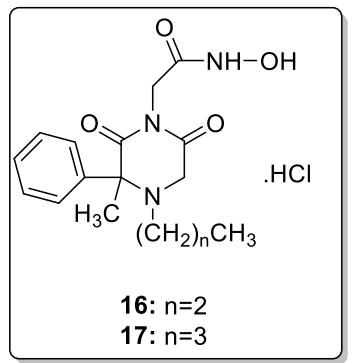

Scheme 2. Reagents and conditions: (a) $\mathrm{NaCN}, \mathrm{CH}_{3}\left(\mathrm{CH}_{2}\right)_{2} \mathrm{NH}_{2} \cdot \mathrm{HCl}$ for $66 \mathrm{n} \mathrm{CH}_{3}\left(\mathrm{CH}_{2}\right)_{3} \mathrm{NH}_{2} . \mathrm{HCl}$ for 67, $\mathrm{DMSO} / \mathrm{H}_{2} \mathrm{O}$ 29:1, rt, 48h; (b) (i) $\mathrm{H}_{2} \mathrm{SO}_{4} 97 \%, \mathrm{CH}_{2} \mathrm{Cl}_{2}$, rt, 24h, (ii) ice and then aq. $\mathrm{NH}_{3} 26 \%$ to pH 7-8, 45\% (66) and $47 \%$ (67) yields over two steps; (c) $\mathrm{BrCH}_{2} \mathrm{CO}_{2} \mathrm{Et}, \mathrm{NaHCO}_{3}, \mathrm{DMF}, 40-43{ }^{\circ} \mathrm{C}, 6 \mathrm{~d}, 57 \%$ for $\mathbf{6 8}, 47 \%$ for $\mathbf{6 9}$; (d) (i) $\left(\mathrm{Me}_{3} \mathrm{Si}\right)_{2} \mathrm{NK}$, THF, 0-5 ${ }^{\circ} \mathrm{C}$ and then rt, $2 \mathrm{~h}$, argon; (ii) $\mathrm{BrCH}_{2} \mathrm{CO}_{2} \mathrm{CH}_{2} \mathrm{C}_{6} \mathrm{H}_{5}$, DMF, rt, 48h, argon, 89\% for 70, 81\% for 71; (e) $\mathrm{H} / 2 / \mathrm{Pd}-$ C, EtOH, 50 psi, rt, 3h, 93\% for 72, 98\% for 73; (f) (i) $\mathrm{CDI}, \mathrm{THF}, 28^{\circ} \mathrm{C}$, $1 \mathrm{~h}$, argon, (ii) $\mathrm{C}_{6} \mathrm{H}_{5} \mathrm{CH}_{2} \mathrm{ONH}_{2} . \mathrm{HCl}, \mathrm{Et}{ }_{3} \mathrm{~N}, 28$ ${ }^{\circ} \mathrm{C}$, 24h and then $45^{\circ} \mathrm{C}, 1 \mathrm{~h}$, argon, 65\% for $\mathbf{7 4}$ and 75 ; (g) (i) as (e), (ii) $\mathrm{HCl}$ in $\mathrm{Et}_{2} \mathrm{O}, 70 \%$ for $\mathbf{1 6}, 68 \%$ for 17.

\subsection{Biological Activity}

The newly synthesized hydroxamic acid derivatives 6-17 were tested against bloodstream form T. brucei in vitro. The $\mathrm{IC}_{50}$ and $\mathrm{IC}_{90}$ values for each compound are summarized in Table 1 . As shown, 10 out of the 12 tested compounds had IC50s values in the low to submicromolar range against $T$. brucei (compounds 6-8 and 11-17) in the free base and hydrochloride forms. The cytotoxicities of the most active compounds against mammalian cells were also determined using the rat skeletal myoblast L6 cells (Table 1.), with most displaying very favorable selective indices.

The initial compound prepared in this 3-alkyl-3-aryl-2,6-DKP-1-acetohydroxamic acid series, 6, exhibited appreciable trypanocidal activity both as free base and hydrochloride salt, with $\mathrm{IC}_{50} \mathrm{~S}$ of 6.97 and $6.61 \mu \mathrm{M}$, respectively. Replacement of the C-3 methyl substituent in the 2,6-DKP sacaffold of 6 with $n$-propyl or $n$-butyl side chains led to the respective more lipophilic C-3 alkyl substituted analogues $\mathbf{7}$ and $\mathbf{8}$. These compounds displayed activities that were comparable to that of 6; the C-3 propyl analogue $7\left(\mathrm{IC}_{50}=7.25 \mu \mathrm{M}\right.$ or $6.93 \mu \mathrm{M}$ as hydrochloride) was almost equipotent to the parent structure 6 , whereas the $\mathrm{C}-3$ butyl counterpart $8\left(\mathrm{IC}_{50}=1.72\right.$ or $1.85 \mu \mathrm{M}$ as hydrochloride) had approximately 4 -fold higher activity than $\mathbf{6}$ and $\mathbf{7}$. However, it is apparent that lengthening of the C-3 alkyl chain in compounds $\mathbf{7}$ and $\mathbf{6}$ by one and three methylene carbons, respectively, boosted potency towards T. brucei to a noteworthy level ( 8 vs 6 and 7). These results demonstrate that the lipophilicity and / or possible steric effects of the C-3 n-alkyl chain influence the trypanocidal activity in this subset of compounds. 
Substitution at para-position of the phenyl moiety in the parent structure 6 by either a lipophilic or hydrophilic electron-withdrawing substituent, such as a fluorine atom or a nitro group was slightly detrimental to activity. The $p$-fluoro substituted analogue 9 was 2.7 and 2 times less potent than the parent $\mathbf{6}$, when these compounds were tested in the free base and hydrochloride salt forms, respectively, while the $p$-nitro congener $\mathbf{1 0}$ proved 1.8-fold less potent than 6 when comparing the $\mathrm{IC}_{50} \mathrm{~S}$ of their corresponding hydrochloride salts. On the other hand, the para-fluoro analogue $\mathbf{9}$ and the para-nitro congener $\mathbf{1 0}$ displayed almost equal activity in the form of their corresponding hydrochloride salts $\left(9, I_{50}=12.9 \mu \mathrm{M} ; 10, I_{50}=11.7 \mu \mathrm{M}\right)$. These findings imply that the observed activity-decrease in compounds $\mathbf{9}$ and $\mathbf{1 0}$ was largely unaffected by the lipophilic or hydrophilic properties, as well as the size of the electron-withdrawing para-substituent.

The addition of a methyl substituent to the N(4)-position of the 2,6-DKP ring [N(4)-methylation] in the parent compounds 6, 7, 9 and 10 appeared to have a favorable effect on the trypanocidal activity and resulted in a 1.512 -fold increase in potency for the respective $N$-methylated analogues 11, 12, 14 and 15 . The greatest improvement in potency was observed with the $N$-methyl analogue $11\left(\mathrm{IC}_{50}=0.59 \mu \mathrm{M}\right.$ or $0.55 \mu \mathrm{M}$ as hydrochloride), which showed a significantly increased trypanocidal activity (12-fold), relative to the unsubstituted parent $\mathbf{6}$. The $\mathrm{N}$-methyl derivatives $\mathbf{1 2}$ and $\mathbf{1 5}$ also gave quite better potencies compared to the corresponding parent structures $\mathbf{7}$ and 10; the C-3 propylated free base $\mathbf{1 2}$ and its hydrochloride (12. $\mathbf{H C l}$ ) had 4.1 and 5.5 times greater trypanocidal activity than $\mathbf{7}$ and $\mathbf{7 . H C l}$, respectively, whereas compound $\mathbf{1 5}$ in the form of its hydrochloride salt $(\mathbf{1 5 .} \mathbf{H C l})$ was 9 -fold more potent than the hydrochloride salt of $\mathbf{1 0}$. However, a slight decrease (1.5-fold) in activity was detected in the case of the C-3 butylated $N$-methyl counterpart 13 upon $N(4)$ methylation of the corresponding parent molecule 8. It is interesting that the effect of the $N(4)$-methylation on the trypanocidal activity within the $N$-methyl derivatives series $\mathbf{1 1 - 1 3}$ seemed to be inversely related to the length of the alkyl side chain at the 3-position of the 2,6-DKP ring, as observed by comparing the analogue pairs $11 / 6,12 / 7$ and $13 / 8$. While the N(4)-methylation of the parent structure 6 led to a significant increase in potency (12-fold) for the C-3 methyl-substituted $N$-methyl analogue 11, it was less beneficial (approximately 4 to 5 -fold increase) and slightly detrimental (1.5-fold decrease) in the cases of the C-3 propyl- and C-3 butyl-substituted $\mathrm{N}$-methyl counterparts 12 and $\mathbf{1 3}$, respectively. These results might be due to the steric interference in the active site.

The marked difference in increasing potency between the $\mathbf{N}$-methylated derivative $\mathbf{1 1}$ and the corresponding $\mathrm{NH}$-analogue 6 prompted the introduction of a longer hydrophobic alkyl substituent such as $n$-propyl or $n$-butyl groups to the N(4)-position of the 2,6-DKP scaffold in parent 6 . These $N(4)$-alkyl substitutions led to significantly effective derivatives which inhibited $T$. brucei growth at submicromolar concentrations. The $N$-propyl derivative $16\left(\mathrm{IC}_{50}=0.47 \mu \mathrm{M}\right)$ and the $n$-butyl counterpart $17\left(\mathrm{IC}_{50}=0.63 \mu \mathrm{M}\right)$ in the form of their hydrochloride salts proved 14- and 10.5 -fold more potent than the hydrochloride salt of the $N$-alkyl free congener 6 , respectively. However, both $N$-alkylated compounds $\mathbf{1 6}$ and $\mathbf{1 7}$ exhibited trypanocidal potencies similar (in the order of $0.5 \mu \mathrm{M}$ ) to the potency of the $N$-methyl derivative 11 . These results indicate that the length and lipophilicity of the $N(4)$-alkyl substituent were not important for potent activity within the group of the acetohydroxamic acid analogues $\mathbf{1 1}$, 16 , and 17 , in which the C-3 alkyl substituent of the 2,6-DKP scaffold is identical.

Notably, the most active compounds in this series $(\mathbf{8}, \mathbf{1 1 - 1 3}$ and 15-17) displayed remarkably low cytotoxicity against mammalian cells (with the exception of compounds 8 and 13), having selectivity indices ranging from 180 (12) to 1180 (11). In particular, the acetohydroxamic acid derivatives 11, 16 and 17, which had the highest activity against $T$. brucei $\left(\mathrm{IC}_{50}=0.55,0.47\right.$ and $0.63 \mu \mathrm{M}$, respectively), displayed the best selectivity with respect to L6 cells.

Table 1. Activity of acetohydroxamic acid analogues 6-17 tested against cultured bloodstream-form T. brucei $(\mathrm{pH}=7.4)$ and cytotoxicity of the most active compounds against cultured rat skeletal myoblast $\mathrm{L} 6$ cells.

\begin{tabular}{|l|l|l|}
\hline & Activity & \\
\hline
\end{tabular}




\begin{tabular}{|c|c|c|c|c|}
\hline \multirow[t]{2}{*}{ Cpds } & \multicolumn{2}{|l|}{ T. brucei } & \multicolumn{2}{|c|}{ Cytotoxicity L6 cells } \\
\hline & $I C_{50}(\mu \mathrm{M})^{\mathrm{a}, \mathrm{b}, \mathrm{e}}$ & $I C_{90}(\mu M)^{a, b, e}$ & $I C_{50}(\mu \mathrm{M})^{\mathrm{c}, \mathrm{e}}$ & $S I^{d, e}$ \\
\hline 6 & $\begin{array}{l}6.97 \pm 1.37 \\
(6.61 \pm 1.65)\end{array}$ & $\begin{array}{l}19.86 \pm 2.99 \\
(17.2 \pm 1.78)\end{array}$ & ND & - \\
\hline 7 & $\begin{array}{l}7.25 \pm 0.25 \\
(6.93 \pm 0.26)\end{array}$ & $10.4 \pm 0.5(8.77 \pm 0.23)$ & ND & - \\
\hline 8 & $\begin{array}{l}1.72 \pm 0.38 \\
(1.85 \pm 0.17)\end{array}$ & $\begin{array}{l}7.54 \pm 0.72 \\
(6.32 \pm 0.65)\end{array}$ & ND $(39 \pm 2)$ & (21) \\
\hline 9 & $\begin{array}{l}19.1 \pm 2.5 \\
(12.9 \pm 0.6)\end{array}$ & $32.9 \pm 1.2(20.3 \pm 0.5)$ & ND & - \\
\hline 10 & $(11.7 \pm 3.6)$ & $(23.2 \pm 2.5)$ & ND & - \\
\hline 11 & $\begin{array}{l}0.59 \pm 0.1 \\
(0.55 \pm 0.06)\end{array}$ & $\begin{array}{l}1.33 \pm 0.17 \\
(1.35 \pm 0.12)\end{array}$ & $\begin{array}{l}698 \pm 69 \\
(549 \pm 40)\end{array}$ & $\begin{array}{l}1180 \\
(1000)\end{array}$ \\
\hline 12 & $\begin{array}{l}1.77 \pm 0.05 \\
(1.27 \pm 0.1)\end{array}$ & $\begin{array}{l}2.23 \pm 0.02 \\
(1.95 \pm 0.06)\end{array}$ & $\begin{array}{l}322 \pm 25 \\
(315 \pm 51)\end{array}$ & $180(248)$ \\
\hline 13 & $\begin{array}{l}2.65 \pm 0.25 \\
(2.97 \pm 0.38)\end{array}$ & $\begin{array}{l}4.71 \pm 0.09 \\
(6.18 \pm 1.11)\end{array}$ & $24 \pm 1$ (ND) & 9 \\
\hline 14 & $\begin{array}{l}8.16 \pm 0.59 \\
(7.86 \pm 0.58)\end{array}$ & $\begin{array}{l}14.5 \pm 0.32 \\
(13.6 \pm 0.38)\end{array}$ & ND & - \\
\hline 15 & $\begin{array}{l}1.22 \pm 0.24 \\
(1.29 \pm 0.16)\end{array}$ & $\begin{array}{l}2.89 \pm 0.24 \\
(2.14 \pm 0.19)\end{array}$ & $\begin{array}{l}373 \pm 18 \\
(397 \pm 21)\end{array}$ & $305(310)$ \\
\hline $16^{d}$ & $(0.47 \pm 0.02)$ & $(1.13 \pm 0.15)$ & $(354 \pm 37)$ & $(750)$ \\
\hline $17^{d}$ & $(0.63 \pm 0.08)$ & $(1.39 \pm 0.03)$ & $(186 \pm 32)$ & (295) \\
\hline
\end{tabular}

${ }^{a}$ Concentrations required to inhibit growth of $T$. brucei by $50 \%$ and $90 \%$, respectively.

${ }^{\mathrm{b}} \mathrm{IC}_{50}$ and $\mathrm{I} \mathrm{C}_{90}$ data are the mean of triplicate experiments \pm SEM.

${ }^{\mathrm{c} C y t o t o x i c i t y ~ w a s ~ d e t e r m i n e d ~ b y ~ e s t a b l i s h i n g ~ t h e ~ c o n c e n t r a t i o n ~ r e q u i r e d ~ t o ~ i n h i b i t ~ g r o w t h ~ o f ~ c u l t u r e d ~ L 6 ~ c e l l s ~}$ by $50 \%\left(\mathrm{IC}_{50}\right)$. Data are the mean of triplicate experiments \pm SEM.

dSelectivity indices were calculated as the ratio of the $\mathrm{IC}_{50}$ for $\mathrm{L} 6$ cells and $T$. brucei.

eData in brackets refer to the respective hydrochloride. ND: Not determined.

\section{Conclusion}

We have developed a novel series of acetohydroxamic acid derivatives that inhibit the bloodstream form $T$. brucei parasite growth with low micromolar or submicromolar $\mathrm{IC}_{50}$ values. These inhibitors were derived from 3-alkyl-3-aryl-2,6-DKP scaffolds by incorporating an acetohydroxamic acid moiety as metal chelating group in their imidic nitrogen atom. Nevertheless, the new class of compounds were found to be less potent than the spiro carbocyclic 2,6-DKP congeners 1-5. ${ }^{x, x}$ The observed decrease in antitrypanosome potency of the new compounds 6-17 might be ascribe to their lower lipophilicity, and unfavorable stereoelectronic factors. Within the $N(4)$-alkyl free analogues 6-8, a C-3 propyl instead of C-3 methyl substitution resulted in an almost equivalent antitrypanosome effect (compare 7 to 6), whereas a significant enhancement in potency was observed upon C-3 butyl substitution (8 vs 6). Substitution at the para-position of the phenyl moiety in the parent 6 by a fluorine atom or nitro group resulted in a slight reduction of activity (compound 9 and 10). 
Introduction of a methyl substituent to the $\mathrm{NH}$-position of the 2,6-DKP ring [N(4)-methylation] in the unsubstituted compounds 6-10 has, in general, a positive influence on the potency against $T$. brucei, as represented by the $N(4)$-methyl analogues $11,12,14$ and $\mathbf{1 5}$. Among the latter compounds, the $N(4)$-methyl derivative 11 was the most potent against trypanosomes, with a submicromolar $\mathrm{IC}_{50}$ value $(0.55 \mu \mathrm{M})$. However, a similar submicromolar T. brucei inhibitory effect (approximately $0.5 \mu \mathrm{M}$ ) was obtained, when the $N(4)$-methyl group in $\mathbf{1 1}$ was replaced by a propyl or butyl $n$-alkyl chain, as in the respective $N(4)$-substituted counterparts 16 and 17 (Table 1.). Importantly, the most potent compounds of this series were found to be highly selective in inhibiting $T$. brucei growth over mammalian cells. Currently, the target of these acetohydroxamic acid derivatives in trypanosomes is unknown. Their submolar potency against $T$. brucei and their relative lack of toxicity to mammalian cells suggests that general metal chelation activity is unlikely. Rather, we would favor a more specific mode of action, analogous to inhibitors of histone deacetylase that have anti-cancer potential, where the mechanism involves binding of the hydroxamate to zinc ions in the catalytic site of the enzyme (Finnin MS, Donigian JR, Cohen A, Richon VM, Rifkind RA, Marks PA, Breslow R, Pavletich NP (1999) Structures of a histone deacetylase homologue bound to the TSA and SAHA inhibitors. Nature 401, 188-193.). Identification of a target in trypanosomes would greatly aid the design of more effective inhibitors.

\section{Materials \& Methods}

\subsection{Chemistry}

\subsubsection{General}

Melting points were determined using a Büchi capillary apparatus and are uncorrected. $1 \mathrm{H}$ and $13 \mathrm{C} \mathrm{NMR}$ spectra were obtained on Bruker MSL $400\left(400 \mathrm{MHz}{ }^{1} \mathrm{H}, 100 \mathrm{MHz}{ }^{13} \mathrm{C}\right)$, Bruker AVANCE III $600\left(600 \mathrm{MHz}{ }^{1} \mathrm{H}, 150 \mathrm{MHz}\right.$ ${ }^{13} \mathrm{C}$ ) and Bruker AVANCE $200\left(50 \mathrm{MHz}{ }^{13} \mathrm{C}\right)$ spectrometers, using $\mathrm{CDCl}_{3}$ or DMSO- $d_{6}$ as solvent. Chemical shifts are reported in $\delta(\mathrm{ppm})$ with tetramethylsilane or solvent (DMSO- $d_{6}$ ) as internal standard. Splitting patterns are designated as s, singlet; $d$, doublet; dd, doublet of doublets; $t$, triplet; td, triplet of doublets; q, quartet; qd, quartet of doublets; $\mathrm{m}$, multiplet; $\mathrm{dm}$, doublet of multiplets; br, broad; $\mathrm{v} b$, very broad; sym, symmetrical. The spectra were recorded at $293 \mathrm{~K}\left(20^{\circ} \mathrm{C}\right)$ unless otherwise specified. Carbon multiplicities were established by DEPT experiments. 2D NMR experiments (HMQC and COSY) were performed for the elucidation of the structures of the newly synthesized compounds. Low-resolution mass spectra were recorded on either an API 2000 LCMS/MS or Thermo-Finnigan AQA model LC-MS system, using positive electrospray ionization mode or Thermo Electron Corporation DSQ mass spectrometer in chemical ionization $(\mathrm{Cl})$ in positive ion mode with methane as $\mathrm{Cl}$ reagent gas or in electron impact (EI). High-resolution mass spectra (HRMS) were determined on a hybrid LTQ-Orbitrap Discovery mass spectrometer under electrospray ionization (ESI) or atmospheric pressure chemical ionization (APCI) in positive ion mode. Analytical thin-layer chromatography (TLC) was conducted on precoated Merck silica gel $60 \mathrm{~F}_{254}$ plates (layer thickness $0.2 \mathrm{~mm}$ ) with the spots visualized by iodine vapors and/or UV light. Column chromatography purification was carried out on silica gel 60 (70-230 and 230-400 mesh ASTM). Elemental analyses (C, H, N) were performed by the Service Central de Microanalyse at CNRS (France) or Department of Microanalysis of NCSR "Democritos" (Greece), and were within $\pm 0.4 \%$ of the calculated values, except where noted (compounds $\mathbf{1 0}$ and $\mathbf{3 3} . \mathbf{H C l}$ ). The purities of the tested compounds were determined by analytical HPLC and elemental analysis. The obtained results correspond to $>95 \%$ purity. Analytical HPLC was performed on a Thermo Finnigan HPLC system (Thermo Finnigan, San Jose, USA) consisting of a SpectraSystem P4000 pump, a SpectraSystem 100 degasser, a SpectraSystem AS3000 autosampler, and a SpectraSystem UV2000 PDA detector, controlled by a SpectraSystem controller. ChromQuest 4.1 software was used for the management of the data. For the HPLC-DAD, a Supelco Analytical Discovery HS C18 (250 mm x $4.6 \mathrm{~mm}, 5.0 \mu \mathrm{m}$ ) 
column was used and the injection volume was $10 \mu \mathrm{L}$. The mobile phase consisted of $\mathrm{H}_{2} \mathrm{O}$ and $1 \%$ acetic acid (solvent A) and acetonitrile (solvent B), and solvent gradient of $A / B$ was $95 / 5$ to $0 / 100$. The analyses were performed at r.t. with a constant flow rate of $1 \mathrm{~mL} / \mathrm{min}$ using a gradient elution of 0-50 min. The commercial reagents were purchased from Alfa Aesar, Sigma-Aldrich and Merck, and were used without further purification except for the benzyl bromoacetate and ethyl bromoacetate. These reagents were purified by distillation prior to use. 4-Methoxybenzyl bromoacetate used, was prepared according to our previously published experimental protocol [6]. O-(4-Methoxybenzyl)hydroxylamine was synthesized according to the literature reported method [9]. Organic solvents used were in the highest purity, and when necessary, were dried by the standard methods. Yields refer to chromatographically pure materials.

\subsubsection{N-[(1-Aminocarbonyl-1-phenyl)ethyl]glycine ethyl ester 23}

To a stirred suspension of sodium cyanide $(1.37 \mathrm{~g}, 27.9 \mathrm{mmol})$ and ethyl glycinate hydrochloride $(3.9 \mathrm{~g}, 27.9$ $\mathrm{mmol})$ in $18 \mathrm{~mL}$ of DMSO- $\mathrm{H}_{2} \mathrm{O}$ 9:1 (v/v), a solution of acetophenone $18(3.2 \mathrm{~g}, 26.6 \mathrm{mmol})$ in DMSO $(36 \mathrm{~mL})$ was added in one portion. The reaction flask was sealed, and the mixture was allowed to react for $48 \mathrm{~h}$ at room temperature with stirring. After this time, the mixture was poured into ice-water $(200 \mathrm{~mL})$ and extracted with $\mathrm{Et}_{2} \mathrm{O}(4 \times 60 \mathrm{~mL})$. The combined organic extracts were washed with water $(2 \times 50 \mathrm{~mL})$, dried, and the solvent was evaporated in vacuo at room temperature to avoid thermal decomposition of the reaction product ( $\alpha$ aminonitrile). The obtained oily residue was dissolved in $\mathrm{CH}_{2} \mathrm{Cl}_{2}(140 \mathrm{~mL})$, cooled in an ice-water bath, and treated dropwise with $\mathrm{H}_{2} \mathrm{SO}_{4} 97 \%$ ( $32 \mathrm{~mL}$ ) under vigorous stirring. The resulting mixture was stirred at room temperature for $24 \mathrm{~h}$, and carefully poured into crashed ice $(170 \mathrm{~g})$. To this two-phase mixture, aqueous $\mathrm{NH}_{3}$ $26 \%$ was added dropwise to $\mathrm{pH}=7-8$ with stirring under cooling (ice-water). The phases were separated, and the aqueous layer was extracted with $\mathrm{CH}_{2} \mathrm{Cl}_{2}(3 \times 100 \mathrm{~mL})$. The combined organic fractions were washed once with water $(100 \mathrm{~mL})$ and once with brine $(100 \mathrm{~mL})$, dried, and evaporated in vacuo. The remaining crude oil was purified by flash column chromatography with $\mathrm{AcOEt}-\mathrm{Et}_{2} \mathrm{O}$ 1:1, as eluent, to afford the title compound $\mathbf{2 3}$ as a white crystalline solid ( $1.35 \mathrm{~g}, 20 \%$ overall yield): $\mathrm{mp} 88-90{ }^{\circ} \mathrm{C}$ (AcOEt/Et $2 \mathrm{O}-n$-pentane); ${ }^{1} \mathrm{H} \mathrm{NMR}\left(400 \mathrm{MHz}, \mathrm{CDCl}_{3}\right)$ $\delta 1.15$ (t, 3H, J=7.1 Hz, $\left.\mathrm{CO}_{2} \mathrm{CH}_{2} \mathrm{CH}_{3}\right), 1.62\left(\mathrm{~s}, 3 \mathrm{H}, \mathrm{CH}_{3}\right), 2.26\left(\mathrm{~s}, 1 \mathrm{H}, \mathrm{NHCH}_{2} \mathrm{CO}_{2} \mathrm{CH}_{2} \mathrm{CH}_{3}\right), 3.11-3.26$ ( q, 2H, J=17.3 $\mathrm{Hz}, \mathrm{NHCH}_{2} \mathrm{CO}_{2} \mathrm{CH}_{2} \mathrm{CH}_{3}$ ), 4.0-4.14 (q, $2 \mathrm{H}, J=7.1 \mathrm{~Hz}, \mathrm{CO}_{2} \mathrm{CH}_{2} \mathrm{CH}_{3}$ ), 6.43 (br s, $1 \mathrm{H}, \mathrm{CONHH}$ ), 6.94 (br s, $1 \mathrm{H}, \mathrm{CONHH}$ ), 7.15-7.51 (m, 5H, aromatic $\mathrm{H}) ;{ }^{13} \mathrm{C} N M R\left(100 \mathrm{MHz}, \mathrm{CDCl}_{3}\right) \delta 14.0\left(\mathrm{CO}_{2} \mathrm{CH}_{2} \mathrm{CH}_{3}\right), 22.6\left(\mathrm{CH}_{3}\right), 45.4$ $\left(\mathrm{NHCH}_{2} \mathrm{CO}_{2} \mathrm{CH}_{2} \mathrm{CH}_{3}\right), 60.9\left(\mathrm{CO}_{2} \mathrm{CH}_{2} \mathrm{CH}_{3}\right), 64.5\left(\mathrm{C}_{6} \mathrm{H}_{5}\left(\mathrm{CH}_{3}\right) \mathrm{CNH}\right), 126.2,127.5,128.4$ (2,3,4,5,6-aromatic $\left.\mathrm{C}\right), 141.6$ (1-aromatic C), $172.1\left(\mathrm{CO}_{2} \mathrm{CH}_{2} \mathrm{CH}_{3}\right), 177.5\left(\mathrm{CONH}_{2}\right)$. Anal. Calcd for $\mathrm{C}_{13} \mathrm{H}_{18} \mathrm{~N}_{2} \mathrm{O}_{3}: \mathrm{C}, 62.38 ; \mathrm{H}, 7.25 ; \mathrm{N}, 11.19$; Found: C, 62.24; H, 7.10; N, 11.24 .

\subsection{3. $\mathrm{N}$-[(1-Aminocarbonyl-1-phenyl)butyl]glycine ethyl ester 24}

Prepared from butyrophenone $\mathbf{1 9}(3.95 \mathrm{~g}, 26.6 \mathrm{mmol}$ ) according to the method described for compound $\mathbf{2 3}$. The crude oil was purified by column chromatography on silica gel with AcOEt- $n$-hexane $1: 1$, as eluent, to afford the title compound 24 as a colorless viscous oil $\left(1.5 \mathrm{~g}, 20 \%\right.$ overall yield); ${ }^{1} \mathrm{H} \mathrm{NMR}\left(400 \mathrm{MHz}, \mathrm{CDCl}_{3}\right) \delta 0.87(\mathrm{t}, 3 \mathrm{H}$, $J=7.3 \mathrm{~Hz}, \mathrm{CH}_{3}\left(\mathrm{CH}_{2}\right)_{2}$ ), 1.06-1.21 (complex m, 5H, $\mathrm{CH}_{3} \mathrm{CH}_{2} \mathrm{CH}_{2}, \mathrm{CO}_{2} \mathrm{CH}_{2} \mathrm{CH}_{3}$ ), 1.94-2.18 (dm, 2H, $\mathrm{CH}_{3} \mathrm{CH}_{2} \mathrm{CH}_{2}$ ), 2.192.40 (br s, $1 \mathrm{H}, \mathrm{NHCH}_{2} \mathrm{CO}_{2} \mathrm{CH}_{2} \mathrm{CH}_{3}$ ), 3.06-3.18 (q, $2 \mathrm{H}, \mathrm{J}=17.0 \mathrm{~Hz}, \mathrm{NHCH}_{2} \mathrm{CO}_{2} \mathrm{CH}_{2} \mathrm{CH}_{3}$ ), 4.05-4.12 (q, $2 \mathrm{H}, \mathrm{J}=7.1 \mathrm{~Hz}$, $\left.\mathrm{CO}_{2} \mathrm{CH}_{2} \mathrm{CH}_{3}\right), 6.03(\mathrm{~s}, 1 \mathrm{H}, \mathrm{CONHH}), 6.93(\mathrm{~s}, 1 \mathrm{H}, \mathrm{CONHH}), 7.15-7.30$ (complex m, 3H, aromatic H), 7.39-7.46 (complex m, $2 \mathrm{H}$, aromatic $\mathrm{H}) ;{ }^{13} \mathrm{C} \mathrm{NMR}\left(50 \mathrm{MHz}, \mathrm{CDCl}_{3}\right) \delta 14.2\left(\mathrm{CH}_{3}\left(\mathrm{CH}_{2}\right)_{2}\right), 14.4\left(\mathrm{CO}_{2} \mathrm{CH}_{2} \mathrm{CH}_{3}\right), 16.7\left(\mathrm{CH}_{3} \mathrm{CH}_{2} \mathrm{CH}_{2}\right)$, $35.8\left(\mathrm{CH}_{3} \mathrm{CH}_{2} \mathrm{CH}_{2}\right), 45.1\left(\mathrm{NHCH}_{2} \mathrm{CO}_{2} \mathrm{CH}_{2} \mathrm{CH}_{3}\right), 61.1\left(\mathrm{CO}_{2} \mathrm{CH}_{2} \mathrm{CH}_{3}\right), 67.4\left(\mathrm{C}_{6} \mathrm{H}_{5}\left(n-\mathrm{C}_{3} \mathrm{H}_{7}\right) \mathrm{CNH}\right), 126.5,127.5,128.6$ (2,3,4,5,6-aromatic C), 141.0 (1-aromatic C), $172.1\left(\mathrm{CO}_{2} \mathrm{CH}_{2} \mathrm{CH}_{3}\right), 176.7\left(\mathrm{CONH}_{2}\right)$; HRMS (ESI): $[\mathrm{M}+\mathrm{H}]^{+}$calcd for $\mathrm{C}_{15} \mathrm{H}_{22} \mathrm{~N}_{2} \mathrm{O}_{3}, 279.1709$, found 279.1705 .

\subsubsection{N-[(1-Aminocarbonyl-1-phenyl)pentyl]glycine ethyl ester 25}

Prepared from valerophenone $\mathbf{2 0}(4.32 \mathrm{~g}, 26.6 \mathrm{mmol})$ according to the method described for compound $\mathbf{2 3}$. The crude oil was purified by flash column chromatography eluting with AcOEt- $n$-hexane 1:2 to 3:1 to afford the title compound 25 as a colorless viscous oil, which solidified on cooling (white crystals, $1.95 \mathrm{~g}, 25 \%$ overall yield): $\mathrm{mp}$ 72-74 ${ }^{\circ} \mathrm{C}\left(\mathrm{Et}_{2} \mathrm{O}-n\right.$-pentane); ${ }^{1} \mathrm{H} \mathrm{NMR}\left(400 \mathrm{MHz}, \mathrm{CDCl}_{3}\right) \delta 0.84\left(\mathrm{t}, 3 \mathrm{H}, \mathrm{J}=7.3 \mathrm{~Hz}, \mathrm{CH}_{3}\left(\mathrm{CH}_{2}\right)_{3}\right), 1.05-1.17$ (complex m, 
$2 \mathrm{H}, \mathrm{CH}_{3} \mathrm{CH}_{2} \mathrm{CH}_{2} \mathrm{CH}_{2}$ ), 1.19 (t, 3H, J=7.1 Hz, $\mathrm{CO}_{2} \mathrm{CH}_{2} \mathrm{CH}_{3}$ ), 1.26-1.39 (complex m, 2H, $\mathrm{CH}_{3} \mathrm{CH}_{2}\left(\mathrm{CH}_{2}\right)_{2}$ ), 1.98-2.23 (dm, $2 \mathrm{H}, \mathrm{CH}_{3}\left(\mathrm{CH}_{2}\right)_{2} \mathrm{CH}_{2}$ ), 2.26-2.48 (br s, $1 \mathrm{H}, \mathrm{NHCH}_{2} \mathrm{CO}_{2} \mathrm{CH}_{2} \mathrm{CH}_{3}$ ), 3.15 ( $\mathrm{s}, 2 \mathrm{H}, \mathrm{NHCH}_{2} \mathrm{CO}_{2} \mathrm{CH}_{2} \mathrm{CH}_{3}$ ), 4.03-4.18 (q, $2 \mathrm{H}$, $\left.J=7.1 \mathrm{~Hz}, \mathrm{CO}_{2} \mathrm{CH}_{2} \mathrm{CH}_{3}\right), 6.64(\mathrm{~s}, 1 \mathrm{H}, \mathrm{CONHH}), 6.96(\mathrm{~s}, 1 \mathrm{H}, \mathrm{CONHH}), 7.16-7.32$ (complex m, 3H, aromatic H), 7.417.50 (complex m, $2 \mathrm{H}$, aromatic $\mathrm{H}$ ); ${ }^{13} \mathrm{C} \mathrm{NMR}\left(50 \mathrm{MHz}, \mathrm{CDCl}_{3}\right) \delta 13.9\left(\mathrm{CH}_{3}\left(\mathrm{CH}_{2}\right)_{3}\right), 14.0\left(\mathrm{CO}_{2} \mathrm{CH}_{2} \mathrm{CH}_{3}\right), 22.8$ $\left(\mathrm{CH}_{3} \mathrm{CH}_{2}\left(\mathrm{CH}_{2}\right)_{2}\right), 25.3\left(\mathrm{CH}_{3} \mathrm{CH}_{2} \mathrm{CH}_{2} \mathrm{CH}_{2}\right), 33.1\left(\mathrm{CH}_{3}\left(\mathrm{CH}_{2}\right)_{2} \mathrm{CH}_{2}\right), 44.9\left(\mathrm{NHCH}_{2} \mathrm{CO}_{2} \mathrm{CH}_{2} \mathrm{CH}_{3}\right), 60.9\left(\mathrm{CO}_{2} \mathrm{CH}_{2} \mathrm{CH}_{3}\right), 67.1$ $\left(\mathrm{C}_{6} \mathrm{H}_{5}\left(n-\mathrm{C}_{4} \mathrm{H}_{9}\right) \mathrm{CNH}\right), 126.3,127.3,128.4$ (2,3,4,5,6-aromatic C), 141.0 (1-aromatic C), $171.9\left(\mathrm{CO}_{2} \mathrm{CH}_{2} \mathrm{CH}_{3}\right), 176.8$ $\left(\mathrm{CONH}_{2}\right)$. Anal. Calcd for $\mathrm{C}_{16} \mathrm{H}_{24} \mathrm{~N}_{2} \mathrm{O}_{3}: \mathrm{C}, 65.72 ; \mathrm{H}, 8.27 ; \mathrm{N}, 9.58$; Found: $\mathrm{C}, 65.95 ; \mathrm{H}, 8.35 ; \mathrm{N}, 9.39$.

\subsubsection{N-[[1-Aminocarbonyl-1-(4-fluorophenyl)]ethyl]glycine ethyl ester 26}

Prepared from 4-fluoroacetophenone $\mathbf{2 1}(3.67 \mathrm{~g}, 26.6 \mathrm{mmol})$ according to the method described for compound 23. The crude oil was purified by column chromatography on silica gel eluting first with AcOEt- $n$-hexane $1: 1$ and then AcOEt to afford the title compound 26 as a light-yellow viscous oil, which solidified on standing $(2.14 \mathrm{~g}$, $30 \%$ overall yield). An analytical sample was obtained as white crystals upon dissolution of the product in $\mathrm{Et}_{2} \mathrm{O}$ and $n$-pentane-mediated precipitation. $\mathrm{Mp} 90-92{ }^{\circ} \mathrm{C} .{ }^{1} \mathrm{H} \mathrm{NMR}\left(600 \mathrm{MHz}, \mathrm{CDCl}_{3}\right) \delta 1.23(\mathrm{t}, 3 \mathrm{H}, J=7.1 \mathrm{~Hz}$, $\left.\mathrm{CO}_{2} \mathrm{CH}_{2} \mathrm{CH}_{3}\right), 1.68\left(\mathrm{~s}, 3 \mathrm{H}, \mathrm{CH}_{3}\right), 2.24\left(\mathrm{~s}, 1 \mathrm{H}, \mathrm{NHCH}_{2} \mathrm{CO}_{2} \mathrm{CH}_{2} \mathrm{CH}_{3}\right), 3.24$ ( s, $\left.2 \mathrm{H}, \mathrm{NHCH}_{2} \mathrm{CO}_{2} \mathrm{CH}_{2} \mathrm{CH}_{3}\right)$, 4.10-4.17 (q, $2 \mathrm{H}$, $\left.J=7.1 \mathrm{~Hz}, \mathrm{CO}_{2} \mathrm{CH}_{2} \mathrm{CH}_{3}\right), 6.18(\mathrm{~s}, 1 \mathrm{H}, \mathrm{CONHH}), 7.01(\mathrm{t}, 2 \mathrm{H}, \mathrm{J}=8.7 \mathrm{~Hz}, 3,5$-aromatic $\mathrm{H}), 7.03(\mathrm{~s}, 1 \mathrm{H}, \mathrm{CONHH}), 7.43-7.49$ $(\sim \mathrm{q}, 2 \mathrm{H}, J=5.3 \mathrm{~Hz}, 2,6$-aromatic $\mathrm{H}) ;{ }^{13} \mathrm{C} \mathrm{NMR}\left(50 \mathrm{MHz}, \mathrm{CDCl}_{3}\right) \delta 14.2\left(\mathrm{CO}_{2} \mathrm{CH}_{2} \mathrm{CH}_{3}\right), 23.1\left(\mathrm{CH}_{3}\right), 45.6$ $\left(\mathrm{NHCH}_{2} \mathrm{CO}_{2} \mathrm{CH}_{2} \mathrm{CH}_{3}\right), 61.3\left(\mathrm{CO}_{2} \mathrm{CH}_{2} \mathrm{CH}_{3}\right), 64.4\left(4-\mathrm{FC}_{6} \mathrm{H}_{4}\left(\mathrm{CH}_{3}\right) \mathrm{CNH}\right), 115.2,115.7$ (d, Jc- $=21.2 \mathrm{~Hz}, 3,5$-aromatic C), 128.2, $128.4\left(\mathrm{~d}, J_{C-F}=8.1 \mathrm{~Hz}, 2,6\right.$-aromatic $\mathrm{C}$ ), 137.6, 137.7 (d, $J_{C-F}=3.3 \mathrm{~Hz}, 1$-aromatic C), 159.8, 164.7 (d, $J_{C-F}=245$ $\mathrm{Hz}$, 4-aromatic C), $172.3\left(\mathrm{CO}_{2} \mathrm{CH}_{2} \mathrm{CH}_{3}\right), 177.4\left(\mathrm{CONH}_{2}\right)$. Anal. Calcd for $\mathrm{C}_{13} \mathrm{H}_{17} \mathrm{FN}_{2} \mathrm{O}_{3}: \mathrm{C}, 58.20 ; \mathrm{H}, 6.39 ; \mathrm{N}, 10.44$; Found: $\mathrm{C}, 58.05 ; \mathrm{H}, 6.45 ; \mathrm{N}, 10.62$.

4.1.6. $\mathrm{N}$-[[1-Aminocarbonyl-1-(4-nitrophenyl)]ethyl]glycine ethyl ester $\mathbf{2 7}$

Prepared from 4-nitroacetophenone $\mathbf{2 2}(4.39 \mathrm{~g}, 26.6 \mathrm{mmol})$ according to the method described for compound 23. The resulting crude oil was purified by column chromatography eluting first with AcOEt- $n$-hexane 1:1 to remove the starting 4-nitroacetophenone 22. Further elution with AcOEt- $n$-hexane 2:1 followed by AcOEt afforded first the respective 2,6-diketopiperazine $\mathbf{4 6}$ (white crystals, $740 \mathrm{mg}, 11 \%$ yield from 22), and then the title compound $\mathbf{2 7}$ as an off-yellow viscous oil, which solidified on standing (4.33 g, 55\% overall yield). An

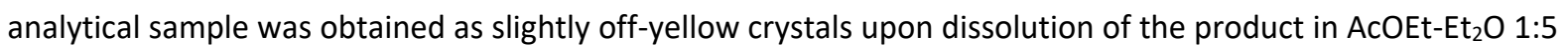
and $n$-pentane-mediated precipitation. $\mathrm{Mp} 83-85{ }^{\circ} \mathrm{C} .{ }^{1} \mathrm{H} \mathrm{NMR}\left(400 \mathrm{MHz}, \mathrm{CDCl}_{3}\right) \delta 1.22(\mathrm{t}, 3 \mathrm{H}, J=7.2 \mathrm{~Hz}$, $\mathrm{CO}_{2} \mathrm{CH}_{2} \mathrm{CH}_{3}$ ), $1.72\left(\mathrm{~s}, 3 \mathrm{H}, \mathrm{CH}_{3}\right.$ ), 2.20-2.46 (br s, $1 \mathrm{H}, \mathrm{NHCH}_{2} \mathrm{CO}_{2} \mathrm{CH}_{2} \mathrm{CH}_{3}$ ), 3.25 ( $\mathrm{s}, 2 \mathrm{H}, \mathrm{NHCH}_{2} \mathrm{CO}_{2} \mathrm{CH}_{2} \mathrm{CH}_{3}$ ), 4.08-4.20 (q, $\left.2 \mathrm{H}, J=7.1 \mathrm{~Hz}, \mathrm{CO}_{2} \mathrm{CH}_{2} \mathrm{CH}_{3}\right), 6.34(\mathrm{~s}, 1 \mathrm{H}, \mathrm{CONHH}), 7.15(\mathrm{~s}, 1 \mathrm{H}, \mathrm{CONHH}), 7.68(\mathrm{~d}, 2 \mathrm{H}, J=8.9 \mathrm{~Hz}, 2,6$-aromatic H), $8.17(\mathrm{~d}, 2 \mathrm{H}, J=8.9 \mathrm{~Hz}, 3,5$-aromatic $\mathrm{H}) ;{ }^{13} \mathrm{C} \mathrm{NMR}\left(50 \mathrm{MHz}, \mathrm{CDCl}_{3}\right) \delta 14.2\left(\mathrm{CO}_{2} \mathrm{CH}_{2} \mathrm{CH}_{3}\right), 23.3\left(\mathrm{CH}_{3}\right), 45.6$ $\left(\mathrm{NHCH}_{2} \mathrm{CO}_{2} \mathrm{CH}_{2} \mathrm{CH}_{3}\right), 61.5\left(\mathrm{CO}_{2} \mathrm{CH}_{2} \mathrm{CH}_{3}\right), 65.0\left(4-\mathrm{NO}_{2} \mathrm{C}_{6} \mathrm{H}_{4}\left(\mathrm{CH}_{3}\right) \mathrm{CNH}\right), 123.7$ (3,5-aromatic C), 127.7 (2,6-aromatic C), 147.3, 149.2 (1,4-aromatic C), $172.0\left(\mathrm{CO}_{2} \mathrm{CH}_{2} \mathrm{CH}_{3}\right), 176.2\left(\mathrm{CONH}_{2}\right)$. Anal. Calcd for $\mathrm{C}_{13} \mathrm{H}_{17} \mathrm{~N}_{3} \mathrm{O}_{5}: \mathrm{C}, 52.87 ; \mathrm{H}$, 5.80; N, 14.23; Found: C, 52.59; H, 5.82; N, 14.52 .

\subsubsection{3-Methyl-2,6-dioxo-3-phenyl-1-piperazineacetic acid benzyl ester $\mathbf{2 8}$}

A stirred solution of the amide-ester derivative $\mathbf{2 3}(1 \mathrm{~g}, 4 \mathrm{mmol})$ in dry THF (40 mL) was cooled in an ice-water bath and treated portionwise with potassium bis(trimethylsilyl)amide $(798 \mathrm{mg}, 4.0 \mathrm{mmol})$ under argon. After 2 $\mathrm{h}$ of stirring at room temperature under argon, the mixture was concentrated to dryness under reduced pressure, and the residue (potassium imidate salt) was dissolved in dry DMF (40 mL). To this solution, benzyl bromoacetate $(962 \mathrm{mg}, 4.2 \mathrm{mmol})$ dissolved in dry DMF (10 mL) was added dropwise, and the mixture was stirred at room temperature for $48 \mathrm{~h}$ under argon. The reaction mixture was then poured into ice-water $(80 \mathrm{~mL})$ and extracted with $\mathrm{Et}_{2} \mathrm{O}(4 \times 60 \mathrm{~mL})$. The combined organic extracts were washed with brine $(2 \times 50 \mathrm{~mL})$, dried $\left(\mathrm{Na}_{2} \mathrm{SO}_{4}\right)$ and concentrated to dryness under reduced pressure. The remaining crude thick oil was purified by flash column chromatography with AcOEt-Et ${ }_{2} \mathrm{O}-n$-hexane $0.5: 1: 1$, as eluent, to afford the title compound $\mathbf{2 8}$ as a pale pink viscous oil (1.13 g, 81\%); ${ }^{1} \mathrm{H}$ NMR $\left(400 \mathrm{MHz}, \mathrm{CDCl}_{3}\right) \delta 1.57\left(\mathrm{~s}, 3 \mathrm{H}, \mathrm{CH}_{3}\right), 2.10-2.39(\mathrm{br} \mathrm{s}, 1 \mathrm{H}, 4-\mathrm{H}), 3.40$ (d, $1 \mathrm{H}, \mathrm{J}=18.5 \mathrm{~Hz}, 5-\mathrm{H}), 3.61$ (d, $1 \mathrm{H}, \mathrm{J}=18.5 \mathrm{~Hz}, 5-\mathrm{H}), 4.45-4.65$ (q, $\mathrm{AB}, 2 \mathrm{H}, \mathrm{J}_{A B}=16.7 \mathrm{~Hz}, \mathrm{CH}_{2} \mathrm{CO}_{2} \mathrm{CH}_{2} \mathrm{Ph}$ ), $5.11(\mathrm{~s}, 2 \mathrm{H}$, 
$\left.\mathrm{CO}_{2} \mathrm{CH}_{2} \mathrm{Ph}\right)$, 7.22-7.42 (m, $10 \mathrm{H}$, aromatic $\left.\mathrm{H}\right) ;{ }^{13} \mathrm{C} \mathrm{NMR}\left(100 \mathrm{MHz}, \mathrm{CDCl}_{3}\right) \delta 28.7\left(\mathrm{CH}_{3}\right), 40.3\left(\mathrm{CH}_{2} \mathrm{CO}_{2} \mathrm{CH}_{2} \mathrm{Ph}\right), 46.0$ (5-C), 62.6 (3-C), $67.3\left(\mathrm{CO}_{2} \mathrm{CH}_{2} \mathrm{Ph}\right), 125.7,128.2,128.4,128.6,128.9,135.1,138.7$ (aromatic C), 167.7 $\left(\mathrm{CO}_{2} \mathrm{CH}_{2} \mathrm{Ph}\right), 171.3,173.6(2,6-\mathrm{C})$; $\mathrm{HRMS}\left(\mathrm{APCl}^{+}\right):[\mathrm{M}+\mathrm{H}]^{+}$calcd for $\mathrm{C}_{20} \mathrm{H}_{20} \mathrm{~N}_{2} \mathrm{O}_{4}, 353.1501$, found 353.1494.

4.1.8. 2,6-Dioxo-3-phenyl-3-propyl-1-piperazineacetic acid benzyl ester 29

Prepared from the amide-ester derivative $24(3.2 \mathrm{~g}, 11.5 \mathrm{mmol})$ following the procedure described for compound 28. The crude yellow solid was purified by column chromatography on silica gel with AcOEt- $n$-hexane $1: 2$, as eluent, to afford the title compound 29 as a slightly off-yellow solid $(3.98 \mathrm{~g}, 91 \%): \mathrm{mp} 63-65^{\circ} \mathrm{C}\left(\mathrm{Et}_{2} \mathrm{O}-n\right.$ pentane); ${ }^{1} \mathrm{H}$ NMR $\left(400 \mathrm{MHz}, \mathrm{CDCl}_{3}\right) \delta 0.88\left(\mathrm{t}, 3 \mathrm{H}, \mathrm{J}=7.2 \mathrm{~Hz}, \mathrm{CH}_{3}\left(\mathrm{CH}_{2}\right)_{2}\right), 1.20-1.38\left(\mathrm{~m}, 2 \mathrm{H}, \mathrm{CH}_{3} \mathrm{CH}_{2} \mathrm{CH}_{2}\right), 1.80-2.13$ (dm, 2H, $\mathrm{CH}_{3} \mathrm{CH}_{2} \mathrm{CH}_{2}$ ), 2.12-2.50 (br s, $\left.1 \mathrm{H}, 4-\mathrm{H}\right), 3.55$ (d, $\left.1 \mathrm{H}, \mathrm{J}=18.4 \mathrm{~Hz}, 5-\mathrm{H}\right), 3.74$ (d, $\left.1 \mathrm{H}, J=18.4 \mathrm{~Hz}, 5-\mathrm{H}\right), 4.51-$ 4.74 (q, $\mathrm{AB}, 2 \mathrm{H}, \mathrm{J}_{\mathrm{AB}}=16.7 \mathrm{~Hz}, \mathrm{CH}_{2} \mathrm{CO}_{2} \mathrm{CH}_{2} \mathrm{Ph}$ ), 5.19 (s, 2H, $\mathrm{CO}_{2} \mathrm{CH}_{2} \mathrm{Ph}$ ), 7.27-7.51 (complex m, 10H, aromatic H); ${ }^{13} \mathrm{C}$ $\operatorname{NMR}\left(50 \mathrm{MHz}, \mathrm{CDCl}_{3}\right) \delta 14.3\left(\mathrm{CH}_{3}\left(\mathrm{CH}_{2}\right)_{2}\right), 17.3\left(\mathrm{CH}_{3} \mathrm{CH}_{2} \mathrm{CH}_{2}\right), 40.4\left(\mathrm{CH}_{2} \mathrm{CO}_{2} \mathrm{CH}_{2} \mathrm{Ph}\right), 44.1\left(\mathrm{CH}_{3} \mathrm{CH}_{2} \mathrm{CH}_{2}\right), 46.0(5-\mathrm{C})$, 65.9 (3-C), $67.4\left(\mathrm{CO}_{2} \mathrm{CH}_{2} \mathrm{Ph}\right), 126.2,128.3,128.4,128.6,128.8,129.0,135.3,137.8$ (aromatic C), 167.9 $\left(\mathrm{CO}_{2} \mathrm{CH}_{2} \mathrm{Ph}\right), 171.3,173.2(2,6-\mathrm{C})$. Anal. Calcd for $\mathrm{C}_{22} \mathrm{H}_{24} \mathrm{~N}_{2} \mathrm{O}_{4}: \mathrm{C}, 69.45 ; \mathrm{H}, 6.36 ; \mathrm{N}, 7.36$; Found: $\mathrm{C}, 69.67 ; \mathrm{H}, 6.42$; $\mathrm{N}, 7.07$.

4.1.9. 3-Butyl-2,6-dioxo-3-phenyl-1-piperazineacetic acid benzyl ester $\mathbf{3 0}$

Prepared from the amide-ester derivative $25(2.5 \mathrm{~g}, 8.55 \mathrm{mmol})$ following the procedure described for compound 28. The crude yellowish solid was purified by flash column chromatography with AcOEt- $n$-hexane 1:2, as eluent, to afford the title compound 30 as a white solid $(2.81 \mathrm{~g}, 83 \%): \mathrm{mp} 92-94{ }^{\circ} \mathrm{C}\left(\mathrm{Et}_{2} \mathrm{O}-n\right.$-pentane); ${ }^{1} \mathrm{H}$ $\operatorname{NMR}\left(400 \mathrm{MHz}, \mathrm{CDCl}_{3}\right) \delta 0.78\left(\mathrm{t}, 3 \mathrm{H}, \mathrm{J}=6.8 \mathrm{~Hz}, \mathrm{CH}_{3}\left(\mathrm{CH}_{2}\right)_{3}\right), 1.10-1.28\left(\mathrm{~m}, 4 \mathrm{H}, \mathrm{CH}_{3} \mathrm{CH}_{2} \mathrm{CH}_{2} \mathrm{CH}_{2}\right), 1.76-2.08(\mathrm{dm}, 2 \mathrm{H}$, $\mathrm{CH}_{3}\left(\mathrm{CH}_{2}\right)_{2} \mathrm{CH}_{2}$ ), 2.09-2.42 (br s, $\left.1 \mathrm{H}, 4-\mathrm{H}\right), 3.47$ (d, $\left.1 \mathrm{H}, \mathrm{J}=18.4 \mathrm{~Hz}, 5-\mathrm{H}\right), 3.66$ (d, $1 \mathrm{H}, \mathrm{J}=18.4 \mathrm{~Hz}, 5-\mathrm{H}$ ), 4.47-4.62 (q, $\left.\mathrm{AB}, 2 \mathrm{H}, \mathrm{J}_{\mathrm{AB}}=16.7 \mathrm{~Hz}, \mathrm{CH}_{2} \mathrm{CO}_{2} \mathrm{CH}_{2} \mathrm{Ph}\right), 5.11\left(\mathrm{~s}, 2 \mathrm{H}, \mathrm{CO}_{2} \mathrm{CH}_{2} \mathrm{Ph}\right), 7.15-7.45\left(\mathrm{~m}, 10 \mathrm{H}\right.$, aromatic H); ${ }^{13} \mathrm{C} \mathrm{NMR} \mathrm{(50} \mathrm{MHz}$, $\left.\mathrm{CDCl}_{3}\right)$ \& $14.0\left(\mathrm{CH}_{3}\left(\mathrm{CH}_{2}\right)_{3}\right), 22.9\left(\mathrm{CH}_{3} \mathrm{CH}_{2}\left(\mathrm{CH}_{2}\right)_{2}\right), 26.0\left(\mathrm{CH}_{3} \mathrm{CH}_{2} \mathrm{CH}_{2} \mathrm{CH}_{2}\right), 40.3\left(\mathrm{CH}_{2} \mathrm{CO}_{2} \mathrm{CH}_{2} \mathrm{Ph}\right), 41.7\left(\mathrm{CH}_{3}\left(\mathrm{CH}_{2}\right)_{2} \mathrm{CH}_{2}\right)$, 45.9 (5-C), 65.8 (3-C), $67.4\left(\mathrm{CO}_{2} \mathrm{CH}_{2} \mathrm{Ph}\right), 126.2,128.3,128.6,128.7,128.9,135.3,137.8$ (aromatic C), 167.8 $\left(\mathrm{CO}_{2} \mathrm{CH}_{2} \mathrm{Ph}\right), 171.3,173.1$ (2,6-C). Anal. Calcd for $\mathrm{C}_{23} \mathrm{H}_{26} \mathrm{~N}_{2} \mathrm{O}_{4}: \mathrm{C}, 70.03 ; \mathrm{H}, 6.64 ; \mathrm{N}, 7.10$; Found: $\mathrm{C}, 70.27 ; \mathrm{H}, 6.80$; $\mathrm{N}, 6.82$.

4.1.10. 3-(4-Fluorophenyl)-3-methyl-2,6-dioxo-1-piperazineacetic acid benzyl ester $\mathbf{3 1}$

Prepared from the amide-ester derivative $26(2.5 \mathrm{~g}, 9.32 \mathrm{mmol})$ following the procedure described for compound 28 . The crude yellow oil was purified by flash column chromatography with AcOEt-n-hexane $1: 2$, as eluent, to afford the title compound 31 as a light-yellow viscous oil $(2.52 \mathrm{~g}, 73 \%):{ }^{1} \mathrm{H} \mathrm{NMR}\left(400 \mathrm{MHz}, \mathrm{CDCl}_{3}\right) \delta$ $1.63\left(\mathrm{~s}, 3 \mathrm{H}, \mathrm{CH}_{3}\right.$ ), 2.86 (br s, $\left.1 \mathrm{H}, 4-\mathrm{H}\right), 3.45$ (d, $1 \mathrm{H}, J=18.6 \mathrm{~Hz}, 5-\mathrm{H}$ ), 3.70 (d, $\left.1 \mathrm{H}, J=18.6 \mathrm{~Hz}, 5-\mathrm{H}\right), 4.54-4.71$ (q, AB, $2 \mathrm{H}, J_{A B}=16.7 \mathrm{~Hz}, \mathrm{CH}_{2} \mathrm{CO}_{2} \mathrm{CH}_{2} \mathrm{Ph}$ ), $5.19\left(\mathrm{~s}, 2 \mathrm{H}, \mathrm{CO}_{2} \mathrm{CH}_{2} \mathrm{Ph}\right), 7.01\left(\mathrm{t}, 2 \mathrm{H}, J=8.7 \mathrm{~Hz}, 3,5-\mathrm{H}\right.$ for $\left.4-\mathrm{FC}_{6} \mathrm{H}_{4}\right), 7.30-7.50$ (complex m, 7H, $\mathrm{C}_{6} \mathrm{H}_{5}, 2,6-\mathrm{H}$ for 4- $\left.\mathrm{FC}_{6} \mathrm{H}_{4}\right) ;{ }^{13} \mathrm{C} \mathrm{NMR}\left(50 \mathrm{MHz}, \mathrm{CDCl}_{3}\right) \delta 29.0\left(\mathrm{CH}_{3}\right), 40.4\left(\mathrm{CH}_{2} \mathrm{CO}_{2} \mathrm{CH}_{2} \mathrm{Ph}\right), 45.9(5-\mathrm{C})$, $62.3(3-\mathrm{C}), 67.5\left(\mathrm{CO}_{2} \mathrm{CH}_{2} \mathrm{Ph}\right), 115.7,116.2\left(\mathrm{~d}, J_{C-F}=21.4 \mathrm{~Hz}, 3,5-\mathrm{C}\right.$ for $\left.4-\mathrm{FC}_{6} \mathrm{H}_{4}\right), 127.7,127.9\left(\mathrm{~d}, J_{C-F}=8.1 \mathrm{~Hz}, 2,6-\mathrm{C}\right.$ for 4- $\left.\mathrm{FC}_{6} \mathrm{H}_{4}\right), 128.4,128.6,128.8\left(2,3,4,5,6-\mathrm{C}\right.$ for $\left.\mathrm{C}_{6} \mathrm{H}_{5}\right), 134.5,134.6\left(\mathrm{~d}, \mathrm{~J}_{\mathrm{C}-\mathrm{F}}=3.2 \mathrm{~Hz}, 1-\mathrm{C}\right.$ for 4- $\left.\mathrm{FC}_{6} \mathrm{H}_{4}\right), 135.2(1-\mathrm{C}$ for $\left.\mathrm{C}_{6} \mathrm{H}_{5}\right), 160.1,165.0$ (d, $\mathrm{J}_{\mathrm{C}-\mathrm{F}}=246 \mathrm{~Hz}$, 4-C for 4- $\left.\mathrm{FC}_{6} \mathrm{H}_{4}\right), 167.8\left(\mathrm{CO}_{2} \mathrm{CH}_{2} \mathrm{Ph}\right), 171.2,173.6(2,6-\mathrm{C})$; HRMS (ESI): $[\mathrm{M}+\mathrm{H}]^{+},[\mathrm{M}+\mathrm{Na}]^{+}$calcd for $\mathrm{C}_{20} \mathrm{H}_{19} \mathrm{FN}_{2} \mathrm{O}_{4}, 371.1407,393.1227$, found 371.1411, 393.1229.

4.1.11. 3-Methyl-3-(4-nitrophenyl)-2,6-dioxo-1-piperazineacetic acid 4-methoxybenzyl ester 32

Prepared from the amide-ester derivative $\mathbf{2 7}(2 \mathrm{~g}, 6.77 \mathrm{mmol})$ following the procedure described for compound 28, but with 4-methoxybenzyl bromoacetate instead of benzyl bromoacetate. The crude yellow solid was purified by column chromatography on silica gel eluting with AcOEt- $n$-hexane 1:1 $\rightarrow 1: 0$ to afford the title compound 32 as an off-yellow solid (2.4 g, 83\%): mp 128-130 ${ }^{\circ} \mathrm{C}$ (AcOEt/Et $2 \mathrm{O}-n$-pentane); ${ }^{1} \mathrm{H}$ NMR (400 MHz, $\left.\mathrm{CDCl}_{3}\right) \delta 1.67\left(\mathrm{~s}, 3 \mathrm{H}, \mathrm{CH}_{3}\right), 2.13-2.38(\mathrm{br} \mathrm{s}, 1 \mathrm{H}, 4-\mathrm{H}), 3.40(\mathrm{~d}, 1 \mathrm{H}, \mathrm{J}=18.7 \mathrm{~Hz}, 5-\mathrm{H}), 3.76(\mathrm{~d}, 1 \mathrm{H}, J=18.7 \mathrm{~Hz}, 5-\mathrm{H}), 3.81$ (s, 3H, OCH 3 ), 4.52-4.68 (q, $\left.\mathrm{AB}, 2 \mathrm{H}, \mathrm{J}_{\mathrm{AB}}=16.7 \mathrm{~Hz}, \mathrm{CH}_{2} \mathrm{CO}_{2} \mathrm{CH}_{2} \mathrm{C}_{6} \mathrm{H}_{4} \mathrm{OCH}_{3}-4\right), 5.11$ (s, $\left.2 \mathrm{H}, \mathrm{CO}_{2} \mathrm{CH}_{2} \mathrm{C}_{6} \mathrm{H}_{4} \mathrm{OCH}_{3}-4\right), 6.88$ (d, $2 \mathrm{H}, \mathrm{J}=8.7 \mathrm{~Hz}, 3,5-\mathrm{H}$ for $\left.4-\mathrm{CH}_{3} \mathrm{OC}_{6} \mathrm{H}_{4}\right), 7.26\left(\mathrm{~d}, 2 \mathrm{H}, \mathrm{J}=8.7 \mathrm{~Hz}, 2,6-\mathrm{H}\right.$ for $\left.4-\mathrm{CH}_{3} \mathrm{OC}_{6} \mathrm{H}_{4}\right), 7.69$ (d, $2 \mathrm{H}, \mathrm{J}=9.0 \mathrm{~Hz}, 2,6-\mathrm{H}$ for $\left.4-\mathrm{NO}_{2} \mathrm{C}_{6} \mathrm{H}_{4}\right), 8.16\left(\mathrm{~d}, 2 \mathrm{H}, \mathrm{J}=9.0 \mathrm{~Hz}, 3,5-\mathrm{H}\right.$ for $\left.4-\mathrm{NO}_{2} \mathrm{C}_{6} \mathrm{H}_{4}\right) ;{ }^{13} \mathrm{C} \mathrm{NMR}\left(50 \mathrm{MHz}, \mathrm{CDCl}_{3}\right) \delta 29.0\left(\mathrm{CH}_{3}\right), 40.6$ $\left(\mathrm{CH}_{2} \mathrm{CO}_{2} \mathrm{CH}_{2} \mathrm{C}_{6} \mathrm{H}_{4} \mathrm{OCH}_{3}-4\right), 45.9$ (5-C), $55.4\left(\mathrm{OCH}_{3}\right), 62.7$ (3-C), $67.6\left(\mathrm{CO}_{2} \mathrm{CH}_{2} \mathrm{C}_{6} \mathrm{H}_{4} \mathrm{OCH}_{3}-4\right), 114.2,124.3,127.2$, 
130.3, 146.2, 148.0, 160.1 (aromatic C), $167.6\left(\mathrm{CO}_{2} \mathrm{CH}_{2} \mathrm{C}_{6} \mathrm{H}_{4} \mathrm{OCH}_{3}-4\right), 170.6,172.7$ (2,6-C). Anal. Calcd for $\mathrm{C}_{21} \mathrm{H}_{21} \mathrm{~N}_{3} \mathrm{O}_{7}: \mathrm{C}, 59.01 ; \mathrm{H}, 4.95 ; \mathrm{N}, 9.83$; Found: $\mathrm{C}, 58.78 ; \mathrm{H}, 4.89 ; \mathrm{N}, 10.02$.

\subsubsection{3-Methyl-2,6-dioxo-3-phenyl-1-piperazineacetic acid 33}

$10 \% \mathrm{Pd}$ on charcoal (132 mg) was added to a solution of benzyl ester $28(1.1 \mathrm{~g}, 3.12 \mathrm{mmol})$ in abs EtOH (78 mL). After $3 \mathrm{~h}$ of shaking under an atmosphere of 50 psi hydrogen at room temperature, the catalyst was removed by filtration and washed with $\mathrm{EtOH}(3 \times 15 \mathrm{~mL})$. The combined filtrates were concentrated to dryness under reduced pressure to afford the title compound $\mathbf{3 3}$ as a white foamy solid, which strongly binds the aforementioned solvent. Removal of the entrapped solvent upon drying at $62-64{ }^{\circ} \mathrm{C}$ under high vacuum $\left(10^{-2}\right.$ $\mathrm{mmHg}$ ) in an Abderhalden apparatus gave 33 as a glass solid (814 mg, almost quantitative yield): ${ }^{1} \mathrm{H}$ NMR (400 $\left.\mathrm{MHz}, \mathrm{CDCl}_{3}\right) \delta 1.67\left(\mathrm{~s}, 3 \mathrm{H}, \mathrm{CH}_{3}\right), 3.48(\mathrm{~d}, 1 \mathrm{H}, \mathrm{J}=18.5 \mathrm{~Hz}, 5-\mathrm{H}), 3.75(\mathrm{~d}, 1 \mathrm{H}, \mathrm{J}=18.5 \mathrm{~Hz}, 5-\mathrm{H}), 4.52-4.70$ (q, AB, $2 \mathrm{H}$, $\left.J_{A B} \simeq 17.0 \mathrm{~Hz}, \mathrm{CH}_{2} \mathrm{CO}_{2} \mathrm{H}\right), 7.0\left(\mathrm{~s}, 2 \mathrm{H}, 4-\mathrm{H}, \mathrm{CO}_{2} \mathrm{H}\right), 7.30-7.48\left(\mathrm{~m}, 5 \mathrm{H}\right.$, aromatic H); ${ }^{13} \mathrm{C} \mathrm{NMR}\left(50 \mathrm{MHz}, \mathrm{CDCl}_{3}\right) \delta 28.4$ $\left(\mathrm{CH}_{3}\right), 40.2\left(\mathrm{CH}_{2} \mathrm{CO}_{2} \mathrm{H}\right), 45.8(5-\mathrm{C}), 62.8(3-\mathrm{C}), 125.7,128.3,129.0$ (2,3,4,5,6-aromatic C), 138.4 (1-aromatic $\left.\mathrm{C}\right)$ $171.1,172.6,173.4\left(\mathrm{CO}_{2} \mathrm{H}, 2,6-\mathrm{C}\right)$. The hydrochloride salt $(33 \cdot \mathrm{HCl})$ was prepared by treating a clear diethyl ether solution of $\mathbf{3 3}$ with saturated solution of $\mathrm{HCl}$ in $\mathrm{Et}_{2} \mathrm{O}$ under ice cooling. The white precipitate was collected by

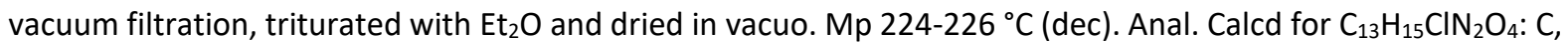
52.27; H, 5.06; N, 9.38; Found: C, 51.85; H, 5.26; N, 9.62.

\subsubsection{2,6-Dioxo-3-phenyl-3-propyl-1-piperazineacetic acid 34}

Prepared from benzyl ester $29(2.46 \mathrm{~g}, 6.47 \mathrm{mmol})$ by catalytic hydrogenolysis $\left(\mathrm{H}_{2} / 10 \% \mathrm{Pd}-\mathrm{C}, 295 \mathrm{mg}\right)$ in abs $\mathrm{EtOH}(160 \mathrm{~mL}$ ) as described for compound 33. The resulting off-white foamy solid was triturated with a diethyl ether- $n$-pentane 1:1 mixture giving the title compound $\mathbf{3 4}$ as white crystals $(1.84 \mathrm{~g}$, 98\%). Recrystallization from $\mathrm{Et}_{2} \mathrm{O}-n$-pentane furnished an analytical sample, which had $\mathrm{mp} 131-133{ }^{\circ} \mathrm{C} .{ }^{1} \mathrm{H} \mathrm{NMR}\left(400 \mathrm{MHz}, \mathrm{CDCl}_{3}\right) \delta 0.88(\mathrm{t}$, $\left.3 \mathrm{H}, J \simeq 7.2 \mathrm{~Hz}, \mathrm{CH}_{3}\left(\mathrm{CH}_{2}\right)_{2}\right), 1.22-1.38\left(\mathrm{~m}, 2 \mathrm{H}, \mathrm{CH}_{3} \mathrm{CH}_{2} \mathrm{CH}_{2}\right), 1.83-2.14\left(\mathrm{dm}, 2 \mathrm{H}, \mathrm{CH}_{3} \mathrm{CH}_{2} \mathrm{CH}_{2}\right), 3.55(\mathrm{~d}, 1 \mathrm{H}, \mathrm{J}=18.4 \mathrm{~Hz}$, 5-H), $3.77(\mathrm{~d}, 1 \mathrm{H}, \mathrm{J}=18.4 \mathrm{~Hz}, 5-\mathrm{H}), 4.50-4.70\left(\mathrm{q}, \mathrm{AB}, 2 \mathrm{H}, \mathrm{J}_{\mathrm{AB}}=17.0 \mathrm{~Hz}, \mathrm{CH}_{2} \mathrm{CO}_{2} \mathrm{H}\right), 6.65\left(\mathrm{~s}, 2 \mathrm{H}, 4-\mathrm{H}, \mathrm{CO}_{2} \mathrm{H}\right), 7.22-7.48$ (m, 5H, aromatic $\mathrm{H}) ;{ }^{13} \mathrm{C} \mathrm{NMR}\left(50 \mathrm{MHz}, \mathrm{CDCl}_{3}\right) \delta 14.3\left(\mathrm{CH}_{3}\left(\mathrm{CH}_{2}\right)_{2}\right), 17.3\left(\mathrm{CH}_{3} \mathrm{CH}_{2} \mathrm{CH}_{2}\right), 40.1\left(\mathrm{CH}_{2} \mathrm{CO}_{2} \mathrm{H}\right), 43.9$ $\left(\mathrm{CH}_{3} \mathrm{CH}_{2} \mathrm{CH}_{2}\right), 45.8$ (5-C), 65.9 (3-C), 126.2, 128.4, 129.0 (2,3,4,5,6-aromatic C), 137.5 (1-aromatic C), 171.3, 173.0 $\left(\mathrm{CO}_{2} \mathrm{H}, 2,6-\mathrm{C}\right)$. Anal. Calcd for $\mathrm{C}_{15} \mathrm{H}_{18} \mathrm{~N}_{2} \mathrm{O}_{4}: \mathrm{C}, 62.06 ; \mathrm{H}, 6.25 ; \mathrm{N}, 9.65$; Found: $\mathrm{C}, 61.79 ; \mathrm{H}, 6.30 ; \mathrm{N}, 9.96$.

\subsubsection{3-Butyl-2,6-dioxo-3-phenyl-1-piperazineacetic acid 35}

Prepared from benzyl ester 30 ( $2.7 \mathrm{~g}, 6.84 \mathrm{mmol})$ by catalytic hydrogenolysis $\left(\mathrm{H}_{2} / 10 \% \mathrm{Pd}-\mathrm{C}, 324 \mathrm{mg}\right)$ in abs EtOHAcOEt 3:1 $(170 \mathrm{~mL})$ as described for compound 33. The resulting glass solid was triturated with $\mathrm{Et}_{2} \mathrm{O}(50 \mathrm{~mL})$ to give the title compound $\mathbf{3 5}$ as an off-white crystalline solid (2 g, 96\%). Recrystallization of specimen of this material from $\mathrm{Et}_{2} \mathrm{O}-n$-pentane furnished an analytical sample as white crystals, mp $121-123{ }^{\circ} \mathrm{C} .{ }^{1} \mathrm{H}$ NMR (400 $\left.\mathrm{MHz}, \mathrm{CDCl}_{3}\right) \delta 0.86\left(\mathrm{t}, 3 \mathrm{H}, J=6.6 \mathrm{~Hz}, \mathrm{CH}_{3}\left(\mathrm{CH}_{2}\right)_{3}\right), 1.18-1.37\left(\mathrm{~m}, 4 \mathrm{H}, \mathrm{CH}_{3} \mathrm{CH}_{2} \mathrm{CH}_{2} \mathrm{CH}_{2}\right), 1.84-2.18$ (sym dm, $2 \mathrm{H}$, $\left.\mathrm{CH}_{3}\left(\mathrm{CH}_{2}\right)_{2} \mathrm{CH}_{2}\right), 3.56(\mathrm{~d}, 1 \mathrm{H}, J=18.5 \mathrm{~Hz}, 5-\mathrm{H}), 3.77$ (d, $\left.1 \mathrm{H}, J=18.5 \mathrm{~Hz}, 5-\mathrm{H}\right), 4.52-4.70$ (q, AB, 2H, JAB $=17.0 \mathrm{~Hz}$, $\left.\mathrm{CH}_{2} \mathrm{CO}_{2} \mathrm{H}\right), 6.80\left(\mathrm{~s}, 2 \mathrm{H}, 4-\mathrm{H}, \mathrm{CO}_{2} \mathrm{H}\right), 7.25-7.52(\mathrm{~m}, 5 \mathrm{H}$, aromatic $\mathrm{H}) ;{ }^{13} \mathrm{C} \mathrm{NMR}\left(50 \mathrm{MHz}, \mathrm{CDCl}_{3}\right) \delta 14.0\left(\mathrm{CH}_{3}\left(\mathrm{CH}_{2}\right)_{3}\right)$, $23.0\left(\mathrm{CH}_{3} \mathrm{CH}_{2}\left(\mathrm{CH}_{2}\right)_{2}\right), 26.1\left(\mathrm{CH}_{3} \mathrm{CH}_{2} \mathrm{CH}_{2} \mathrm{CH}_{2}\right), 40.1\left(\mathrm{CH}_{2} \mathrm{CO}_{2} \mathrm{H}\right), 41.6\left(\mathrm{CH}_{3}\left(\mathrm{CH}_{2}\right)_{2} \mathrm{CH}_{2}\right), 45.8$ (5-C), 65.9 (3-C), 126.2, 128.3, 129.0 (2,3,4,5,6-aromatic C), 137.6 (1-aromatic C), 171.3, 173.0, $173.4\left(\mathrm{CO}_{2} \mathrm{H}, 2,6-\mathrm{C}\right)$. Anal. Calcd for $\mathrm{C}_{16} \mathrm{H}_{20} \mathrm{~N}_{2} \mathrm{O}_{4}$ : C, 63.14; $\mathrm{H}, 6.62 ; \mathrm{N}, 9.20$; Found: $\mathrm{C}, 62.90 ; \mathrm{H}, 6.68 ; \mathrm{N}, 9.48$.

\subsubsection{3-(4-Fluorophenyl)-3-methyl-2,6-dioxo-1-piperazineacetic acid 36}

Prepared from benzyl ester $31(2.47 \mathrm{~g}, 6.67 \mathrm{mmol})$ by catalytic hydrogenolysis $\left(\mathrm{H}_{2} / 10 \% \mathrm{Pd}-\mathrm{C}, 296 \mathrm{mg}\right)$ in abs $\mathrm{EtOH}(167 \mathrm{~mL})$ as described for compound 33 . The obtained white foamy solid strongly binds the hydrogenation solvent. Removal of the entrapped solvent as in $\mathbf{3 3}$ gave the title compound $\mathbf{3 6}$ as a white amorphous solid (1.86 $\mathrm{g}$, almost quantitative yield). A small quantity of this material was dissolved in AcOEt-Et ${ }_{2} \mathrm{O}$ 1:1, and the clear solution was concentrated to dryness in vacuo. Trituration of the residue with a diethyl ether- $n$-pentane 1:1 mixture provided an analytical sample as white crystals. Mp 150-153 ${ }^{\circ} \mathrm{C} .{ }^{1} \mathrm{H}$ NMR (400 MHz, DMSO-d $\left.d_{6}\right) \delta 1.50(\mathrm{~s}$, $\left.3 \mathrm{H}, \mathrm{CH}_{3}\right), 3.16$ (d, $\left.1 \mathrm{H}, \mathrm{J}=18.4 \mathrm{~Hz}, 5-\mathrm{H}\right), 3.62\left(\mathrm{~d}, 1 \mathrm{H}, \mathrm{J}=18.4 \mathrm{~Hz}, 5-\mathrm{H}\right.$ ), 3.25-4.25 (v br s, 2H, 4-H, $\mathrm{CO}_{2} \mathrm{H}$ ), 4.30-4.45 (q, $\mathrm{AB}, 2 \mathrm{H}, \mathrm{J}_{\mathrm{AB}} \simeq 17.3 \mathrm{~Hz}, \mathrm{CH}_{2} \mathrm{CO}_{2} \mathrm{H}$ ), 7.19 (t like, $2 \mathrm{H}, \mathrm{J}=7.3,8.4 \mathrm{~Hz}, 3,5$-aromatic $\mathrm{H}$ ), 7.47 (t like, $2 \mathrm{H}, \mathrm{J}=5.3,7.5 \mathrm{~Hz}, 2,6-$ 
aromatic $\mathrm{H}) ;{ }^{13} \mathrm{C}$ NMR $(50 \mathrm{MHz}$, DMSO-d $) \delta 28.3\left(\mathrm{CH}_{3}\right), 40.2\left(\mathrm{CH}_{2} \mathrm{CO}_{2} \mathrm{H}\right), 45.6(5-\mathrm{C}), 61.6(3-\mathrm{C}), 115.2,115.7(\mathrm{~d}$, $J_{C-F}=21.2 \mathrm{~Hz}, 3,5$-aromatic C), 128.0, 128.2 (d, $J_{C-F}=7.9 \mathrm{~Hz}, 2,6$-aromatic C), 136.2 (1-aromatic C), 159.2, 164.1 (d, $J_{C-F}=243 \mathrm{~Hz}, 4$-aromatic C), $169.1\left(\mathrm{CO}_{2} \mathrm{H}\right), 171.3,173.5(2,6-\mathrm{C})$. Anal. Calcd for $\mathrm{C}_{13} \mathrm{H}_{13} \mathrm{FN}_{2} \mathrm{O}_{4}: \mathrm{C}, 55.71 ; \mathrm{H}, 4.68 ; \mathrm{N}$, 10.0; Found: C, 55.98; $\mathrm{H}, 4.46 ; \mathrm{N}, 9.66$.

\subsubsection{3-Methyl-3-phenyl-2,6-piperazinedione 42}

The amide-ester derivative $23(500 \mathrm{mg}, 2 \mathrm{mmol})$ was subjected to treatment with potassium bis(trimethylsilyl)amide (399 $\mathrm{mg}, 2.0 \mathrm{mmol})$ in dry THF $(20 \mathrm{~mL})$ as described in 28 . The reaction was then quenched by adding trifluoroacetic acid ( $230 \mathrm{mg}, 2 \mathrm{mmol}, 1$ equiv), and the solvent was evaporated to dryness under reduced pressure. Purification of the residual viscous oil by column chromatography on silica gel with AcOEt-Et ${ }_{2} \mathrm{O}-n$-hexane 1:1:0.5, as eluent, afforded the title compound $\mathbf{4 2}$ as a white crystalline solid (380 mg, 93\%): $\mathrm{mp} 131-133{ }^{\circ} \mathrm{C}$ (AcOEt/Et $2 \mathrm{O}-n$-pentane) (lit $\left.{ }^{[10]} \mathrm{mp} 133-133.5^{\circ} \mathrm{C}\right) ;{ }^{1} \mathrm{H} \mathrm{NMR}\left(400 \mathrm{MHz}, \mathrm{CDCl}_{3}\right) \delta 1.63(\mathrm{~s}, 3 \mathrm{H}$, $\left.\mathrm{CH}_{3}\right), 2.31(\mathrm{~s}, 1 \mathrm{H}, 4-\mathrm{H}), 3.38(\mathrm{~d}, 1 \mathrm{H}, \mathrm{J}=18.6 \mathrm{~Hz}, 5-\mathrm{H}), 3.62(\mathrm{~d}, 1 \mathrm{H}, \mathrm{J}=18.6 \mathrm{~Hz}, 5-\mathrm{H}), 7.27-7.53$ (complex m, 5H, aromatic $\mathrm{H}), 8.79(\mathrm{~s}, 1 \mathrm{H}, 1-\mathrm{H}) ;{ }^{13} \mathrm{C} \mathrm{NMR}\left(50 \mathrm{MHz}, \mathrm{CDCl}_{3}\right) \delta 28.1\left(\mathrm{CH}_{3}\right), 45.9(5-\mathrm{C}), 62.3(3-\mathrm{C}), 125.7,128.4,129.2$ (2,3,4,5,6-aromatic C), 138.6 (1-aromatic C) 172.9, $174.5(2,6-C)$. Anal. Calcd for $\mathrm{C}_{11} \mathrm{H}_{12} \mathrm{~N}_{2} \mathrm{O}_{2}: \mathrm{C}, 64.69 ; \mathrm{H}, 5.92 ; \mathrm{N}$, 13.72; Found: C, 64.66; $\mathrm{H}, 5.82 ; \mathrm{N}, 13.88$.

\subsubsection{3-Phenyl-3-propyl-2,6-piperazinedione 43}

Prepared from the amide-ester derivative $24(1.17 \mathrm{~g}, 4.2 \mathrm{mmol})$ in the same manner as for $\mathbf{4 2}$. The resulting crude oil was purified by column chromatography on silica gel with AcOEt- $n$-hexane $1: 1$, as eluent, to afford the title compound 43 as a glass oil $(930 \mathrm{mg}, 95 \%)$. Crystallization of this product material upon treatment with a diethyl ether- $n$-pentane 1:1 mixture, and subsequent recrystallization ( $\mathrm{Et}_{2} \mathrm{O}-n$-pentane) gave a white crystalline solid, which melted at $80-82^{\circ} \mathrm{C}$ (lit ${ }^{[10]} \mathrm{mp} 75-77.5^{\circ} \mathrm{C}$ ) after drying at room temperature in vacuo. This solid product strongly binds $\mathrm{Et}_{2} \mathrm{O}$ as indicated by its ${ }^{1} \mathrm{H}$ NMR spectrum. Removal of the crystal solvent upon drying at 62-64 ${ }^{\circ} \mathrm{C}$ under high vacuum $\left(10^{-2} \mathrm{mmHg}\right)$ in an Abderhalden apparatus restored the solid material to the initial glass oil state. ${ }^{1} \mathrm{H}$ NMR $\left(400 \mathrm{MHz}, \mathrm{CDCl}_{3}\right) \delta 0.8\left(\mathrm{t}, 3 \mathrm{H}, \mathrm{J}=7.3 \mathrm{~Hz}, \mathrm{CH}_{3}\left(\mathrm{CH}_{2}\right)_{2}\right), 1.10-1.32\left(\mathrm{~m}, 2 \mathrm{H}, \mathrm{CH}_{3} \mathrm{CH}_{2} \mathrm{CH}_{2}\right), 1.77-$ $2.0\left(\mathrm{dm}, 2 \mathrm{H}, \mathrm{CH}_{3} \mathrm{CH}_{2} \mathrm{CH}_{2}\right.$ ), 2.09-2.32 (br s, $\left.1 \mathrm{H}, 4-\mathrm{H}\right), 3.39$ (d, $\left.1 \mathrm{H}, J=18.5 \mathrm{~Hz}, 5-\mathrm{H}\right), 3.58(\mathrm{~d}, 1 \mathrm{H}, J=18.5 \mathrm{~Hz}, 5-\mathrm{H}), 7.17-$ $7.47(\mathrm{~m}, 5 \mathrm{H}$, aromatic $\mathrm{H}), 8.53(\mathrm{~s}, 1 \mathrm{H}, 1-\mathrm{H}) ;{ }^{13} \mathrm{C} \mathrm{NMR}\left(50 \mathrm{MHz}, \mathrm{CDCl}_{3}\right) \delta 14.3\left(\mathrm{CH}_{3}\left(\mathrm{CH}_{2}\right)_{2}\right), 17.3\left(\mathrm{CH}_{3} \mathrm{CH}_{2} \mathrm{CH}_{2}\right), 43.2$ $\left(\mathrm{CH}_{3} \mathrm{CH}_{2} \mathrm{CH}_{2}\right), 45.7$ (5-C), 65.3 (3-C), 126.1, 128.3, 129.0 (2,3,4,5,6-aromatic C), 137.4 (1-aromatic C), 172.7, 174.0 (2,6-C); $\mathrm{ES}^{+} \mathrm{MS}: \mathrm{m} / \mathrm{z} 233.2[\mathrm{M}+\mathrm{H}]^{+}$.

\subsubsection{3-Butyl-3-phenyl-2,6-piperazinedione 44}

Prepared from the amide-ester derivative $25(2.24 \mathrm{~g}, 7.66 \mathrm{mmol})$ in the same manner as for $\mathbf{4 2}$. The resulting crude oil was purified by flash column chromatography eluting with AcOEt- $n$-hexane 1:2 to afford the title compound 44 as a slightly off-white solid (1.81 g, 96\%). To obtain an analytical sample, a small quantity of this material was recrystallized from $\mathrm{Et}_{2} \mathrm{O}-n$-pentane giving a cotton like solid, which melted at $72-74{ }^{\circ} \mathrm{C}$ after drying at room temperature in vacuo. This solid material strongly binds the recrystallization solvents as indicated by its ${ }^{1} \mathrm{H}$ NMR spectrum. Removal of the crystal solvents upon drying at $62-64{ }^{\circ} \mathrm{C}$ under high vacuum $\left(10^{-2} \mathrm{mmHg}\right)$ provided a white crystalline solid, which had $\mathrm{mp} 79-82{ }^{\circ} \mathrm{C} .{ }^{1} \mathrm{H} \mathrm{NMR}\left(400 \mathrm{MHz}, \mathrm{CDCl}_{3}\right) \delta 0.86(\mathrm{t}, 3 \mathrm{H}, \mathrm{J}=6.8 \mathrm{~Hz}$, $\left.\mathrm{CH}_{3}\left(\mathrm{CH}_{2}\right)_{3}\right), 1.16-1.37\left(\mathrm{~m}, 4 \mathrm{H}, \mathrm{CH}_{3} \mathrm{CH}_{2} \mathrm{CH}_{2} \mathrm{CH}_{2}\right), 1.83-2.13\left(\mathrm{dm}, 2 \mathrm{H}, \mathrm{CH}_{3}\left(\mathrm{CH}_{2}\right)_{2} \mathrm{CH}_{2}\right), 2.18-2.36(\mathrm{br} \mathrm{s}, 1 \mathrm{H}, 4-\mathrm{H}), 3.47$ (d, $1 \mathrm{H}, \mathrm{J}=18.1 \mathrm{~Hz}, 5-\mathrm{H}), 3.66(\mathrm{~d}, 1 \mathrm{H}, \mathrm{J}=18.5 \mathrm{~Hz}, 5-\mathrm{H}), 7.28-7.53\left(\mathrm{~m}, 5 \mathrm{H}\right.$, aromatic H), $7.96(\mathrm{~s}, 1 \mathrm{H}, 1-\mathrm{H}) ;{ }^{13} \mathrm{C} \mathrm{NMR}(50$ $\left.\mathrm{MHz}, \mathrm{CDCl}_{3}\right) \delta 14.0\left(\mathrm{CH}_{3}\left(\mathrm{CH}_{2}\right)_{3}\right), 22.9\left(\mathrm{CH}_{3} \mathrm{CH}_{2}\left(\mathrm{CH}_{2}\right)_{2}\right), 26.1\left(\mathrm{CH}_{3} \mathrm{CH}_{2} \mathrm{CH}_{2} \mathrm{CH}_{2}\right), 40.9\left(\mathrm{CH}_{3}\left(\mathrm{CH}_{2}\right)_{2} \mathrm{CH}_{2}\right), 45.8(5-\mathrm{C}), 65.3$ (3-C), 126.1, 128.3, 129.1 (2,3,4,5,6-aromatic C), 137.5 (1-aromatic C), 172.4, 173.8 (2,6-C). Anal. Calcd for $\mathrm{C}_{14} \mathrm{H}_{18} \mathrm{~N}_{2} \mathrm{O}_{2}$ : C, 68.27; $\mathrm{H}, 7.37$; N, 11.37; Found: C, 68.42; $\mathrm{H}, 7.64 ; \mathrm{N}, 11.09$.

\subsubsection{3-(4-Fluorophenyl)-3-methyl-2,6-piperazinedione 45}

Prepared from the amide-ester derivative $\mathbf{2 6}(500 \mathrm{mg}, 1.86 \mathrm{mmol})$ in the same manner as for $\mathbf{4 2}$. The resulting crude solid was purified by flash column chromatography eluting with AcOEt- $n$-hexane 2:1 to afford the title compound 45 as a white crystalline solid (380 mg, 92\%): mp 151-153 ${ }^{\circ} \mathrm{C}$. (AcOEt/Et ${ }_{2} \mathrm{O} 1: 20-n$-pentane); ${ }^{1} \mathrm{H}$ NMR $\left(600 \mathrm{MHz}, \mathrm{DMSO}-d_{6}\right) \delta 1.43\left(\mathrm{~s}, 3 \mathrm{H}, \mathrm{CH}_{3}\right), 2.98-3.04$ (q, $\left.1 \mathrm{H}, J=10.7 \mathrm{~Hz}, 5-\mathrm{H}\right), 3.40-3.45$ (dd, $1 \mathrm{H}, J=4.5,18.3 \mathrm{~Hz}, 5-$ 
H), 3.79-3.83 (dd, $1 \mathrm{H}, J=4.5,10.6 \mathrm{~Hz}, 4-\mathrm{H}), 7.21(\sim \mathrm{t}, 2 \mathrm{H}, J=8.8 \mathrm{~Hz}, 3,5$-aromatic H), 7.40-7.47 ( q, $2 \mathrm{H}, J=5.5 \mathrm{~Hz}$, 2,6-aromatic H), $11.07(\mathrm{~s}, 1 \mathrm{H}, 1-\mathrm{H}) ;{ }^{13} \mathrm{C}$ NMR (50 MHz, DMSO-d $) \delta 27.7\left(\mathrm{CH}_{3}\right), 45.4(5-\mathrm{C}), 60.8(3-\mathrm{C}), 115.4,115.8$ (d, $J_{C-F}=21.3 \mathrm{~Hz}, 3,5$-aromatic C), 127.5, 127.7 (d, $J_{C-F}=8.1 \mathrm{~Hz}, 2,6$-aromatic C), 136.3 (1-aromatic C), $159.1,164.0$ (d, $J_{C-F}=242 \mathrm{~Hz}$, 4-aromatic C), 173.1, $174.8(2,6-C)$. Anal. Calcd for $\mathrm{C}_{11} \mathrm{H}_{11} \mathrm{FN}_{2} \mathrm{O}_{2}$ : C, 59.45; H, 4.99; N, 12.61; Found: C, 59.41; H, 4.89; N, 12.76.

4.1.20. 3-Methyl-3-(4-nitrophenyl)-2,6-piperazinedione 46

Prepared from the amide-ester derivative $\mathbf{2 7}(1 \mathrm{~g}, 3.39 \mathrm{mmol})$ in the same manner as for $\mathbf{4 2}$. The resulting crude solid was purified by column chromatography on silica gel with AcOEt- $n$-hexane $2: 1$, as eluent, to afford the title compound 46 as an off-yellow solid (770 mg, 91\%): $\mathrm{mp} 211-213{ }^{\circ} \mathrm{C}$. (MeOH-Et $\left.2 \mathrm{O}\right) ;{ }^{1} \mathrm{H} \mathrm{NMR}\left(400 \mathrm{MHz}, \mathrm{DMSO}-d_{6}\right)$ $\delta 1.47$ (s, 3H, $\mathrm{CH}_{3}$ ), 2.98-3.11 (q, $\left.1 \mathrm{H}, J=10.8 \mathrm{~Hz}, 5-\mathrm{H}\right), 3.42-3.52$ (dd, $1 \mathrm{H}, J=4.5,18.4 \mathrm{~Hz}, 5-\mathrm{H}$ ), 3.92-4.02 (dd, $1 \mathrm{H}$, $J=4.4,10.7 \mathrm{~Hz}, 4-\mathrm{H}), 7.69(\mathrm{~d}, 2 \mathrm{H}, J=8.8 \mathrm{~Hz}, 2,6$-aromatic $\mathrm{H}), 8.25$ (d, 2H, J=8.0 Hz, 3,5-aromatic H), $11.22(\mathrm{~s}, 1 \mathrm{H}$, 1-H); ${ }^{13} \mathrm{C}$ NMR (50 MHz, DMSO- $\left.d_{6}\right) \delta 27.4\left(\mathrm{CH}_{3}\right), 45.4(5-\mathrm{C}), 61.3(3-\mathrm{C}), 124.0$ (3,5-aromatic C), 127.0 (2,6-aromatic C), 147.1, 148.0 (1,4-aromatic C), 172.8, 174.1 (2,6-C). Anal. Calcd for $\mathrm{C}_{11} \mathrm{H}_{11} \mathrm{~N}_{3} \mathrm{O}_{4}$ : C, 53.01; $\mathrm{H}, 4.45 ; \mathrm{N}, 16.86$; Found: $\mathrm{C}, 52.88 ; \mathrm{H}, 4.61 ; \mathrm{N}, 17.04$.

\subsubsection{3,4-Dimethyl-3-phenyl-2,6-piperazinedione 47}

A solution of compound 42 (721 mg, $3.53 \mathrm{mmol}$ ) and aqueous formaldehyde $37 \%(2.2 \mathrm{~mL})$ in methanol (22 $\mathrm{mL})$ was stirred at room temperature for $3 \mathrm{~h}$, and $\mathrm{NaCNBH}_{3}(398 \mathrm{mg}, 6.33 \mathrm{mmol}$ ) was then added in one portion. After 20 min of stirring, the $\mathrm{pH}$ of the reaction mixture was adjusted to 6-7 by dropwise addition of acetic acid. Stirring was continued for $4 \mathrm{~h}$ at room temperature with occasional addition of acetic acid to maintain the $\mathrm{pH}$ at 6-7. Methanol was removed by evaporation in vacuo, and the residue was treated with water (10 $\mathrm{mL})$ followed by $1 \mathrm{~N}$ aq $\mathrm{NaOH}$ and solid $\mathrm{Na}_{2} \mathrm{CO}_{3}$ until the $\mathrm{pH}$ was adjusted to 8 . Subsequently the mixture was extracted with $\mathrm{CH}_{2} \mathrm{Cl}_{2}(3 \times 35 \mathrm{~mL})$, and the combined organic extracts were washed with brine $(2 \times 35 \mathrm{~mL})$, dried $\left(\mathrm{Na}_{2} \mathrm{SO}_{4}\right)$ and concentrated to dryness under reduced pressure. The remaining viscous oil solidified after a few minutes of standing. The resulting crude solid was purified over a column of flash silica eluting with AcOEt- $n$ hexane-Et ${ }_{2} \mathrm{O}$ 1:1:0.5 to afford the title compound 47 as a white crystalline solid (593 $\mathrm{mg}, 77 \%$ ): $\mathrm{mp} 127-129{ }^{\circ} \mathrm{C}$. (Et ${ }_{2} \mathrm{O}-n$-pentane); ${ }^{1} \mathrm{H}$ NMR $\left(400 \mathrm{MHz}, \mathrm{CDCl}_{3}\right) \delta 1.63\left(\mathrm{~s}, 3 \mathrm{H}, 3-\mathrm{CH}_{3}\right), 2.52\left(\mathrm{~s}, 3 \mathrm{H}, 4-\mathrm{CH}_{3}\right), 3.27-3.61(\mathrm{q}, \mathrm{AB}, 2 \mathrm{H}$, $\left.J_{A B}=18.2 \mathrm{~Hz}, 5-\mathrm{H}\right), 7.27-7.47(\mathrm{~m}, 5 \mathrm{H}$, aromatic $\mathrm{H}), 8.79($ br s, $1 \mathrm{H}, 1-\mathrm{H}) ;{ }^{13} \mathrm{C} \mathrm{NMR}\left(50 \mathrm{MHz}, \mathrm{CDCl}_{3}\right) \delta 23.5\left(3-\mathrm{CH}_{3}\right)$, $38.3\left(4-\mathrm{CH}_{3}\right), 54.9$ (5-C), 67.7 (3-C), 126.0, 128.3, 129.0 (2,3,4,5,6-aromatic C), 140.5 (1-aromatic C), 171.4, 174.1 (2,6-C). Anal. Calcd for $\mathrm{C}_{12} \mathrm{H}_{14} \mathrm{~N}_{2} \mathrm{O}_{2}$ : C, 66.04; $\mathrm{H}, 6.47 ; \mathrm{N}, 12.84$; Found: $\mathrm{C}, 65.91 ; \mathrm{H}, 6.62 ; \mathrm{N}, 12.62$.

4.1.22. 4-Methyl-3-phenyl-3-propyl-2,6-piperazinedione 48

Prepared by reductive methylation of diketopiperazine 43 (920 mg, $3.96 \mathrm{mmol}$ ) as described for compound 47. The crude thick oil was purified by column chromatography on silica gel with AcOEt- $n$-hexane $1: 3$, as eluent, to afford a colorless viscous oil, which solidified on standing (white crystals, $860 \mathrm{mg}, 88 \%$ yield): $\mathrm{mp} 116-118{ }^{\circ} \mathrm{C}$ (Et ${ }_{2} \mathrm{O}-n$-pentane); ${ }^{1} \mathrm{H}$ NMR $\left(400 \mathrm{MHz}, \mathrm{CDCl}_{3}\right) \delta 0.75$ (t, 3H, J=7.4 Hz, $\left.\mathrm{CH}_{3}\left(\mathrm{CH}_{2}\right)_{2}\right), 1.02-1.20\left(\mathrm{~m}, 2 \mathrm{H}, \mathrm{CH}_{3} \mathrm{CH}_{2} \mathrm{CH}_{2}\right)$, 1.77-2.08 (dm, 2H, $\mathrm{CH}_{3} \mathrm{CH}_{2} \mathrm{CH}_{2}$ ), $2.43\left(\mathrm{~s}, 3 \mathrm{H}, 4-\mathrm{CH}_{3}\right), 3.21-3.66\left(\mathrm{q}, \mathrm{AB}, 2 \mathrm{H}, J_{A B}=18.2 \mathrm{~Hz}, 5-\mathrm{H}\right), 7.17-7.36(\mathrm{~m}, 5 \mathrm{H}$, aromatic $\mathrm{H}), 8.82(\mathrm{~s}, 1 \mathrm{H}, 1-\mathrm{H}) ;{ }^{13} \mathrm{C} \mathrm{NMR}\left(50 \mathrm{MHz}, \mathrm{CDCl}_{3}\right) \delta 14.3\left(\mathrm{CH}_{3}\left(\mathrm{CH}_{2}\right)_{2}\right), 16.2\left(\mathrm{CH}_{3} \mathrm{CH}_{2} \mathrm{CH}_{2}\right), 38.1\left(4-\mathrm{CH}_{3}\right), 38.3$ $\left(\mathrm{CH}_{3} \mathrm{CH}_{2} \mathrm{CH}_{2}\right.$ ), 55.1 (5-C), 70.0 (3-C), 126.9, 128.2, 128.7 (2,3,4,5,6-aromatic C), 136.9 (1-aromatic C), 171.4, 174.0 (2,6-C). Anal. Calcd for $\mathrm{C}_{14} \mathrm{H}_{18} \mathrm{~N}_{2} \mathrm{O}_{2}$ : C, 68.27; $\mathrm{H}, 7.37 ; \mathrm{N}, 11.37$; Found: C, 68.41; H, 7.21; N, 11.51.

4.1.23. 3-Butyl-4-methyl-3-phenyl-2,6-piperazinedione 49

Prepared by reductive methylation of diketopiperazine 44 (900 mg, $3.65 \mathrm{mmol}$ ) as described for compound 47. The crude yellowish thick oil was purified over a column of flash silica eluting with AcOEt- $n$-hexane 1:4 to afford the title compound $\mathbf{4 9}$ as a colorless viscous oil, which solidified on standing under cooling (white crystals, 810 $\mathrm{mg}$, 85\% yield): $\mathrm{mp} 67-69^{\circ} \mathrm{C}$. (Et $\mathrm{Et}_{2} \mathrm{O}-n$-pentane); ${ }^{1} \mathrm{H} \mathrm{NMR}\left(400 \mathrm{MHz}, \mathrm{CDCl}_{3}\right) \delta 0.82\left(\mathrm{t}, 3 \mathrm{H}, \mathrm{J} \simeq 7.0 \mathrm{~Hz}, \mathrm{CH}_{3}\left(\mathrm{CH}_{2}\right)_{3}\right)$, 1.05-1.31 (m, 4H, $\left.\mathrm{CH}_{3} \mathrm{CH}_{2} \mathrm{CH}_{2} \mathrm{CH}_{2}\right)$, 1.89-2.15 (dm, 2H, $\left.\mathrm{CH}_{3}\left(\mathrm{CH}_{2}\right)_{2} \mathrm{CH}_{2}\right), 2.50\left(\mathrm{~s}, 3 \mathrm{H}, 4-\mathrm{CH}_{3}\right), 3.26-3.76(\mathrm{q}, \mathrm{AB}, 2 \mathrm{H}$, $\left.J_{A B}=18.2 \mathrm{~Hz}, 5-\mathrm{H}\right), 7.30-7.52(\mathrm{~m}, 5 \mathrm{H}$, aromatic $\mathrm{H}), 8.48(\mathrm{~s}, 1 \mathrm{H}, 1-\mathrm{H}) ;{ }^{13} \mathrm{C} \mathrm{NMR}\left(50 \mathrm{MHz}, \mathrm{CDCl}_{3}\right) \delta 14.0\left(\mathrm{CH}_{3}\left(\mathrm{CH}_{2}\right)_{3}\right)$, $22.9\left(\mathrm{CH}_{3} \mathrm{CH}_{2}\left(\mathrm{CH}_{2}\right)_{2}\right), 24.8\left(\mathrm{CH}_{3} \mathrm{CH}_{2} \mathrm{CH}_{2} \mathrm{CH}_{2}\right), 35.8\left(\mathrm{CH}_{3}\left(\mathrm{CH}_{2}\right)_{2} \mathrm{CH}_{2}\right), 38.1\left(4-\mathrm{CH}_{3}\right), 55.1(5-\mathrm{C}), 70.1(3-\mathrm{C}), 126.9,128.3$, 
128.7 (2,3,4,5,6-aromatic C), 136.8 (1-aromatic C), 171.2, 173.9 (2,6-C). Anal. Calcd for $\mathrm{C}_{15} \mathrm{H}_{20} \mathrm{~N}_{2} \mathrm{O}_{2}: \mathrm{C}, 69.20 ; \mathrm{H}$, 7.74; N, 10.76; Found: C, 69.32; H, 7.63; N, 10.98.

\subsubsection{3-(4-Fluorophenyl)-3,4-dimethyl-2,6-piperazinedione 50}

Prepared by reductive methylation of diketopiperazine $45(1.05 \mathrm{~g}, 4.73 \mathrm{mmol})$ as described for compound 47. The crude yellowish thick oil was purified by column chromatography on silica gel with AcOEt-n-hexane $1: 3$, as eluent, to afford the title compound as a colorless viscous oil, which solidified on standing (white crystals, 765 $\mathrm{mg}, 69 \%$ yield): $\mathrm{mp} 116-118{ }^{\circ} \mathrm{C}$. (Et ${ }_{2} \mathrm{O}-n$-pentane); ${ }^{1} \mathrm{H} \mathrm{NMR}\left(600 \mathrm{MHz}, \mathrm{CDCl}_{3}\right) \delta 1.60$ (s, 3H, 3- $\left.\mathrm{CH}_{3}\right), 2.48(\mathrm{~s}, 3 \mathrm{H}, 4-$ $\mathrm{CH}_{3}$ ), 3.30-3.57 (q, $\mathrm{AB}, 2 \mathrm{H}, J_{A B}=18.1 \mathrm{~Hz}, 5-\mathrm{H}$ ), 7.04 (t like, $2 \mathrm{H}, \mathrm{J}=8.6 \mathrm{~Hz}, 3,5$-aromatic H), 7.40-7.45 (q like, $2 \mathrm{H}, \mathrm{J}=5.2$ $\mathrm{Hz}, 2,6$-aromatic $\mathrm{H}), 8.64(\mathrm{~s}, 1 \mathrm{H}, 1-\mathrm{H}) ;{ }^{13} \mathrm{C} \mathrm{NMR}\left(50 \mathrm{MHz}, \mathrm{CDCl}_{3}\right) \delta 23.0\left(3-\mathrm{CH}_{3}\right), 38.3\left(4-\mathrm{CH}_{3}\right), 54.7(5-\mathrm{C}), 67.1(3-$ C), 115.7, 116.1 (d, $J_{C-F}=21.4 \mathrm{~Hz}, 3,5$-aromatic C), 127.8, 128.0 (d, $J_{C-F}=7.9 \mathrm{~Hz}, 2$,6-aromatic C), 136.3 (1-aromatic C), $160.0,165.0$ (d, $\mathrm{J}_{C-\mathrm{F}}=246 \mathrm{~Hz}$, 4-aromatic C), 171.1, 174.0 (2,6-C). Anal. Calcd for $\mathrm{C}_{12} \mathrm{H}_{13} \mathrm{FN}_{2} \mathrm{O}_{2}: \mathrm{C}, 61.01 ; \mathrm{H}, 5.55$; $\mathrm{N}, 11.86$; Found: $\mathrm{C}, 61.18 ; \mathrm{H}, 5.45 ; \mathrm{N}, 12.08$.

\subsubsection{3,4-Dimethyl-3-(4-nitrophenyl)-2,6-piperazinedione 51}

Prepared by reductive methylation of diketopiperazine $46(1.06 \mathrm{~g}, 4.25 \mathrm{mmol})$ in THF-MeOH 1:1 $(50 \mathrm{~mL})$ as described for compound $\mathbf{4 7}$. The crude viscous was purified by column chromatography on silica gel with AcOEt$n$-hexane 1:4 to $1: 2$ to afford the title compound $\mathbf{5 1}$ as a white crystalline solid (925 mg, 83\%): $\mathrm{mp} 136-138^{\circ} \mathrm{C}$. (AcOEt/Et $2 \mathrm{O}-n$-pentane); ${ }^{1} \mathrm{H}$ NMR $\left(400 \mathrm{MHz}, \mathrm{CDCl}_{3}\right) \delta 1.65$ (s, 3H, 3- $\left.\mathrm{CH}_{3}\right), 2.50$ (s, 3H, 4-CH3), 3.36-3.57 (q, AB, $\left.2 \mathrm{H}, J_{A B}=18.1 \mathrm{~Hz}, 5-\mathrm{H}\right), 7.69(\mathrm{~d}, 2 \mathrm{H}, \mathrm{J}=9.0 \mathrm{~Hz}, 2,6$-aromatic H), $8.23(\mathrm{~d}, 2 \mathrm{H}, J=9.0 \mathrm{~Hz}, 3,5$-aromatic H), 8.55 (s, $1 \mathrm{H}$, 1-H); $\left.{ }^{13} \mathrm{C} \mathrm{NMR} \mathrm{(50} \mathrm{MHz,} \mathrm{CDCl}\right) \delta 22.4\left(3-\mathrm{CH}_{3}\right), 38.3\left(4-\mathrm{CH}_{3}\right), 54.7$ (5-C), 67.5 (3-C), 124.3 (3,5-aromatic C), 127.4 (2,6-aromatic C), 148.0 (1,4-aromatic C), 170.2, 173.0 (2,6-C). Anal. Calcd for $\mathrm{C}_{12} \mathrm{H}_{13} \mathrm{~N}_{3} \mathrm{O}_{4}$ : C, 54.75; $\mathrm{H}, 4.98 ; \mathrm{N}$, 15.96; Found: C, 54.59; H, 4.82; N, 16.14.

\subsubsection{3,4-Dimethyl-2,6-dioxo-3-phenyl-1-piperazineacetic acid benzyl ester $\mathbf{5 2}$}

A stirred solution of diketopiperazine $47(1.13 \mathrm{~g}, 5.18 \mathrm{mmol})$ in dry DMF $(30 \mathrm{~mL})$ was treated portionwise with sodium hydride (149 mg, $6.22 \mathrm{mmol})$. After $1 \mathrm{~h}$ of stirring at room temperature under argon, benzyl bromoacetate $(1.25 \mathrm{~g}, 5.43 \mathrm{mmol})$ dissolved in dry DMF $(6 \mathrm{~mL})$ was added dropwise. Stirring was continued at room temperature for $48 \mathrm{~h}$ under argon, and the reaction mixture was then poured into ice-water $(75 \mathrm{~mL})$ and extracted with ethyl acetate $(4 \times 60 \mathrm{~mL})$. The combined organic extracts were washed with brine $(2 \times 60 \mathrm{~mL})$, dried $\left(\mathrm{Na}_{2} \mathrm{SO}_{4}\right)$, and concentrated to dryness in vacuo. The residual thick oil was purified by flash column chromatography eluting with AcOEt- $n$-hexane 1:2 to afford the title compound $\mathbf{5 2}$ as a light-yellow viscous oil, which solidified on standing under cooling ( $1.62 \mathrm{~g}, 85 \%)$. An analytical sample was obtained as white crystals upon dissolution of the product in $\mathrm{Et}_{2} \mathrm{O}$ and $n$-pentane-mediated precipitation. $\mathrm{Mp} 90-92{ }^{\circ} \mathrm{C} .{ }^{1} \mathrm{H} \mathrm{NMR}(400 \mathrm{MHz}$, $\left.\mathrm{CDCl}_{3}\right) \delta 1.65\left(\mathrm{~s}, 3 \mathrm{H}, 3-\mathrm{CH}_{3}\right), 2.54\left(\mathrm{~s}, 3 \mathrm{H}, 4-\mathrm{CH}_{3}\right), 3.37-3.72\left(\mathrm{q}, \mathrm{AB}, 2 \mathrm{H}, \mathrm{J}_{A B}=18.0 \mathrm{~Hz}, 5-\mathrm{H}\right), 4.59-4.75$ (q, AB, $2 \mathrm{H}$, $J_{A B}=16.8 \mathrm{~Hz}, \mathrm{CH}_{2} \mathrm{CO}_{2} \mathrm{CH}_{2} \mathrm{Ph}$ ), $5.20\left(\mathrm{~s}, 2 \mathrm{H}, \mathrm{CO}_{2} \mathrm{CH}_{2} \mathrm{Ph}\right.$ ), 7.25-7.47 (complex m, $10 \mathrm{H}$, aromatic $\mathrm{H}$ ); ${ }^{13} \mathrm{C} \mathrm{NMR}(100 \mathrm{MHz}$, $\left.\mathrm{CDCl}_{3}\right) \delta 24.4\left(3-\mathrm{CH}_{3}\right), 38.1\left(4-\mathrm{CH}_{3}\right), 40.3\left(\mathrm{CH}_{2} \mathrm{CO}_{2} \mathrm{CH}_{2} \mathrm{Ph}\right), 55.1(5-\mathrm{C}), 67.5\left(\mathrm{CO}_{2} \mathrm{CH}_{2} \mathrm{Ph}\right), 68.0(3-\mathrm{C}), 126.1,128.3$, 128.5, 128.6, 128.7, 129.0, 135.2, 140.7 (aromatic C), $167.8\left(\mathrm{CO}_{2} \mathrm{CH}_{2} \mathrm{Ph}\right), 170.4,173.7$ (2,6-C). Anal. Calcd for $\mathrm{C}_{21} \mathrm{H}_{22} \mathrm{~N}_{2} \mathrm{O}_{4}$ : C, 68.84; $\mathrm{H}, 6.05 ; \mathrm{N}, 7.65$; Found: $\mathrm{C}, 69.24 ; \mathrm{H}, 6.43 ; \mathrm{N}, 8.01$.

4.1.27. 4-Methyl-2,6-dioxo-3-phenyl-3-propyl-1-piperazineacetic acid benzyl ester 53

Prepared from diketopiperazine $\mathbf{4 8}(1.15 \mathrm{~g}, 4.67 \mathrm{mmol})$ following the procedure described for compound $\mathbf{5 2}$. The crude oil was purified by column chromatography on silica gel with AcOEt-petroleum ether $\left(44-60{ }^{\circ} \mathrm{C}\right) 1: 6$, as eluent, to afford the title compound $\mathbf{5 3}$ as a slightly off-yellow viscous oil $(1.35 \mathrm{~g}, 75 \%):{ }^{1} \mathrm{H} \mathrm{NMR} \mathrm{(400} \mathrm{MHz}$, $\left.\mathrm{CDCl}_{3}\right) \delta 0.75\left(\mathrm{t}, 3 \mathrm{H}, \mathrm{J} \simeq 7.3 \mathrm{~Hz}, \mathrm{CH}_{3}\left(\mathrm{CH}_{2}\right)_{2}\right), 1.04-1.15\left(\mathrm{~m}, 2 \mathrm{H}, \mathrm{CH}_{3} \mathrm{CH}_{2} \mathrm{CH}_{2}\right), 1.78-2.09\left(\mathrm{dm}, 2 \mathrm{H}, \mathrm{CH}_{3} \mathrm{CH}_{2} \mathrm{CH}_{2}\right), 2.42$ (s, 3H, 4- $\mathrm{CH}_{3}$ ), 3.28-3.71 (q, $\left.\mathrm{AB}, 2 \mathrm{H}, \mathrm{J}_{\mathrm{AB}}=18.1 \mathrm{~Hz}, 5-\mathrm{H}\right), 4.47-4.63\left(\mathrm{q}, \mathrm{AB}, 2 \mathrm{H}, \mathrm{J}_{\mathrm{AB}}=16.7 \mathrm{~Hz}, \mathrm{CH}_{2} \mathrm{CO}_{2} \mathrm{CH}_{2} \mathrm{Ph}\right.$ ), 5.06-5.16 (q, $\mathrm{AB}, 2 \mathrm{H}, J_{A B}=12.3 \mathrm{~Hz}, \mathrm{CO}_{2} \mathrm{CH}_{2} \mathrm{Ph}$ ), 7.16-7.34 (complex m, $10 \mathrm{H}$, aromatic $\mathrm{H}$ ); ${ }^{13} \mathrm{C} \mathrm{NMR} \mathrm{(50} \mathrm{MHz,} \mathrm{CDCl}_{3}$ ) $\delta 14.3$ $\left(\mathrm{CH}_{3}\left(\mathrm{CH}_{2}\right)_{2}\right), 16.3\left(\mathrm{CH}_{3} \mathrm{CH}_{2} \mathrm{CH}_{2}\right), 37.8\left(4-\mathrm{CH}_{3}\right), 39.0\left(\mathrm{CH}_{3} \mathrm{CH}_{2} \mathrm{CH}_{2}\right), 40.4\left(\mathrm{CH}_{2} \mathrm{CO}_{2} \mathrm{CH}_{2} \mathrm{Ph}\right), 55.1(5-\mathrm{C}), 67.4\left(\mathrm{CO}_{2} \mathrm{CH}_{2} \mathrm{Ph}\right)$, 70.3 (3-C), 127.0, 128.2, 128.4, 128.6, 128.7, 135.2, 136.9 (aromatic C), $167.8\left(\mathrm{CO}_{2} \mathrm{CH}_{2} \mathrm{Ph}\right), 170.4,173.6$ (2,6-C); HRMS (ESI): $[\mathrm{M}+\mathrm{H}]^{+}$calcd for $\mathrm{C}_{23} \mathrm{H}_{26} \mathrm{~N}_{2} \mathrm{O}_{4}, 395.1971$, found 395.1967. 


\subsubsection{3-Butyl-4-methyl-2,6-dioxo-3-phenyl-1-piperazineacetic acid benzyl ester $\mathbf{5 4}$}

Prepared from diketopiperazine $\mathbf{4 9}(1.45 \mathrm{~g}, 5.57 \mathrm{mmol})$ following the procedure described for compound $\mathbf{5 2}$. The crude oil was purified by flash column chromatography eluting with AcOEt- $n$-hexane 1:3 to afford the title compound 54 as a colorless viscous oil (1.97 g, 87\%): $\left.{ }^{1} \mathrm{H} \mathrm{NMR} \mathrm{(600} \mathrm{MHz,} \mathrm{CDCl} 3\right) \delta 0.75\left(\mathrm{t}, 3 \mathrm{H}, \mathrm{J}=7.2 \mathrm{~Hz}, \mathrm{CH}_{3}\left(\mathrm{CH}_{2}\right)_{3}\right)$, 1.04-1.20 (dm, $\left.4 \mathrm{H}, \mathrm{CH}_{3} \mathrm{CH}_{2} \mathrm{CH}_{2} \mathrm{CH}_{2}\right), 1.85-2.09\left(\mathrm{dm}, 2 \mathrm{H}, \mathrm{CH}_{3}\left(\mathrm{CH}_{2}\right)_{2} \mathrm{CH}_{2}\right), 2.42$ (s, 3H, 4- $\left.\mathrm{CH}_{3}\right), 3.30-3.69$ (q, $\mathrm{AB}, 2 \mathrm{H}$, $J_{A B}=18.1 \mathrm{~Hz}, 5-\mathrm{H}$ ), 4.49-4.61 (q, $\mathrm{AB}, 2 \mathrm{H}, \mathrm{J}_{A B}=16.7 \mathrm{~Hz}, \mathrm{CH}_{2} \mathrm{CO}_{2} \mathrm{CH}_{2} \mathrm{Ph}$ ), 5.08-5.14 (q, $\mathrm{AB}, 2 \mathrm{H}, \mathrm{J}_{A B}=12.2 \mathrm{~Hz}, \mathrm{CO}_{2} \mathrm{CH}_{2} \mathrm{Ph}$ ), 7.16-7.32 (complex m, $10 \mathrm{H}$, aromatic $\mathrm{H}$ ); ${ }^{13} \mathrm{C} \mathrm{NMR}\left(50 \mathrm{MHz}, \mathrm{CDCl}_{3}\right) \delta 14.0\left(\mathrm{CH}_{3}\left(\mathrm{CH}_{2}\right)_{3}\right), 22.9\left(\mathrm{CH}_{3} \mathrm{CH}_{2}\left(\mathrm{CH}_{2}\right)_{2}\right), 24.9$ ( $\left.\mathrm{CH}_{3} \mathrm{CH}_{2} \mathrm{CH}_{2} \mathrm{CH}_{2}\right), 36.5\left(\mathrm{CH}_{3}\left(\mathrm{CH}_{2}\right)_{2} \mathrm{CH}_{2}\right), 37.8\left(4-\mathrm{CH}_{3}\right), 40.4\left(\mathrm{CH}_{2} \mathrm{CO}_{2} \mathrm{CH}_{2} \mathrm{Ph}\right), 55.1$ (5-C), $67.4\left(\mathrm{CO}_{2} \mathrm{CH}_{2} \mathrm{Ph}\right), 70.3$ (3-C), 127.0, 128.2, 128.4, 128.6, 128.7, 135.2, 136.8 (aromatic C), $167.8\left(\mathrm{CO}_{2} \mathrm{CH}_{2} \mathrm{Ph}\right), 170.4,173.7$ (2,6-C); HRMS (ESI): $[\mathrm{M}+\mathrm{H}]^{+}$calcd for $\mathrm{C}_{24} \mathrm{H}_{28} \mathrm{~N}_{2} \mathrm{O}_{4}, 409.2127$, found 409.2118 .

\subsubsection{3-(4-Fluorophenyl)-3,4-dimethyl-2,6-dioxo-1-piperazineacetic acid benzyl ester $\mathbf{5 5}$}

Prepared from piperazine $\mathbf{5 0}(1.53 \mathrm{~g}, 6.48 \mathrm{mmol})$ following the procedure described for compound $\mathbf{5 2}$. The crude oil was purified by column chromatography on silica gel with AcOEt- $n$-hexane 1:2, as eluent, to afford the title compound 55 as a colorless viscous oil $(1.78 \mathrm{~g}, 71 \%):{ }^{1} \mathrm{H} N M R\left(400 \mathrm{MHz}, \mathrm{CDCl}_{3}\right) \delta 1.62\left(\mathrm{~s}, 3 \mathrm{H}, 3-\mathrm{CH}_{3}\right), 2.50(\mathrm{~s}, 3 \mathrm{H}$, 4- $\mathrm{CH}_{3}$ ), 3.37-3.68 (q, $\mathrm{AB}, 2 \mathrm{H}, \mathrm{J}_{A B}=18.0 \mathrm{~Hz}, 5-\mathrm{H}$ ), 4.56-4.73 (q, $\mathrm{AB}, 2 \mathrm{H}, \mathrm{J}_{A B}=16.8 \mathrm{~Hz}, \mathrm{CH}_{2} \mathrm{CO}_{2} \mathrm{CH}_{2} \mathrm{Ph}$ ), 5.19 (s, $2 \mathrm{H}$, $\mathrm{CO}_{2} \mathrm{CH}_{2} \mathrm{Ph}$ ), 7.0 (t, $2 \mathrm{H}, \mathrm{J}=8.7 \mathrm{~Hz}, 3,5-\mathrm{H}$ for 4- $\mathrm{FC}_{6} \mathrm{H}_{4}$ ), 7.30-7.44 (complex m, 7H, $\mathrm{C}_{6} \mathrm{H}_{5}, 2,6-\mathrm{H}$ for 4- $\mathrm{FC}_{6} \mathrm{H}_{4}$ ); ${ }^{13} \mathrm{C} \mathrm{NMR}$ $\left(50 \mathrm{MHz}, \mathrm{CDCl}_{3}\right) \delta 24.0\left(3-\mathrm{CH}_{3}\right), 38.1\left(4-\mathrm{CH}_{3}\right), 40.4\left(\mathrm{CH}_{2} \mathrm{CO}_{2} \mathrm{CH}_{2} \mathrm{Ph}\right), 54.9(5-\mathrm{C}), 67.5\left(\mathrm{CO}_{2} \mathrm{CH}_{2} \mathrm{Ph}\right), 69.1$ (3-C), 115.6, $116.0\left(\mathrm{~d}, J_{C-F}=21.3 \mathrm{~Hz}, 3,5-\mathrm{C}\right.$ for $\left.4-\mathrm{FC}_{6} \mathrm{H}_{4}\right), 128.0,128.1$ (d, $J_{C-F}=7.9 \mathrm{~Hz}, 2,6-\mathrm{C}$ for $\left.4-\mathrm{FC}_{6} \mathrm{H}_{4}\right), 128.5,128.7,128.8$ $\left(2,3,4,5,6-\mathrm{C}\right.$ for $\left.\mathrm{C}_{6} \mathrm{H}_{5}\right), 135.2,136.5$ (1-C for 4- $\mathrm{FC}_{6} \mathrm{H}_{4}$ and $\left.\mathrm{C}_{6} \mathrm{H}_{5}\right), 160.0,164.9$ (d, $\mathrm{J}_{\mathrm{C}-\mathrm{F}}=246 \mathrm{~Hz}, 4-\mathrm{C}$ for 4- $\left.\mathrm{FC}_{6} \mathrm{H}_{4}\right), 167.7$ $\left(\mathrm{CO}_{2} \mathrm{CH}_{2} \mathrm{Ph}\right), 170.1,173.5$ (2,6-C); HRMS (ESI): [M+H] $]^{+}$calcd for $\mathrm{C}_{21} \mathrm{H}_{21} \mathrm{FN}_{2} \mathrm{O}_{4}, 385.1564$, found 385.1576 .

\subsubsection{3,4-Dimethyl-3-(4-nitrophenyl)-2,6-dioxo-1-piperazineacetic acid 4-methoxybenzyl ester 56}

Sodium hydride (92 mg, $3.83 \mathrm{mmol}$ ) was added in portions to a stirred solution of diketopiperazine 51 (840 mg, $3.19 \mathrm{mmol})$ in dry DMF $(20 \mathrm{~mL})$, and the mixture was left stirring for $10 \mathrm{~min}$ at room temperature under argon. In this time window, the mixture color changed from light yellow to blue-red. Then, 4-methoxybenzyl bromoacetate $(868 \mathrm{mg}, 3.35 \mathrm{mmol}$ ) dissolved in dry DMF $(5 \mathrm{~mL})$ was added dropwise, and the mixture was stirred at room temperature for $48 \mathrm{~h}$ under argon. The reaction was then worked up in the same way described for the preparation of $\mathbf{5 2}$. The resulting orange thick oil was purified by column chromatography on silica gel eluting with AcOEt-n-hexane 1:3 and then $1: 2$ to afford the title compound $\mathbf{5 6}$ as a clear, yellow-orange viscous oil (1.18 g, 84\%): ${ }^{1} \mathrm{H} \mathrm{NMR}\left(400 \mathrm{MHz}, \mathrm{CDCl}_{3}\right) \delta 1.65\left(\mathrm{~s}, 3 \mathrm{H}, 3-\mathrm{CH}_{3}\right), 2.51$ (s, 3H, 4- $\left.\mathrm{CH}_{3}\right), 3.40-3.64$ (q, AB, 2H, $\mathrm{J}_{A B}=18.1$ $\mathrm{Hz}, 5-\mathrm{H}), 3.80$ (s, $\left.3 \mathrm{H}, \mathrm{OCH}_{3}\right), 4.55-4.69$ (q, $\mathrm{AB}, 2 \mathrm{H}, J_{A B}=16.7 \mathrm{~Hz}, \mathrm{CH}_{2} \mathrm{CO}_{2} \mathrm{CH}_{2} \mathrm{C}_{6} \mathrm{H}_{4} \mathrm{OCH}_{3}-4$ ), 5.11 (s, $2 \mathrm{H}$, $\left.\mathrm{CO}_{2} \mathrm{CH}_{2} \mathrm{C}_{6} \mathrm{H}_{4} \mathrm{OCH}_{3}-4\right), 6.88\left(\mathrm{~d}, 2 \mathrm{H}, \mathrm{J}=8.7 \mathrm{~Hz}, 3,5-\mathrm{H}\right.$ for $\left.4-\mathrm{CH}_{3} \mathrm{OC}_{6} \mathrm{H}_{4}\right), 7.26$ (d, $2 \mathrm{H}, J=8.7 \mathrm{~Hz}, 2,6-\mathrm{H}$ for $4-\mathrm{CH}_{3} \mathrm{OC}_{6} \mathrm{H}_{4}$ ), $7.65\left(\mathrm{~d}, 2 \mathrm{H}, \mathrm{J}=9.0 \mathrm{~Hz}, 2,6-\mathrm{H}\right.$ for $\left.4-\mathrm{NO}_{2} \mathrm{C}_{6} \mathrm{H}_{4}\right), 8.15\left(\mathrm{~d}, 2 \mathrm{H}, \mathrm{J}=9.0 \mathrm{~Hz}, 3,5-\mathrm{H}\right.$ for $\left.4-\mathrm{NO}_{2} \mathrm{C}_{6} \mathrm{H}_{4}\right) ;{ }^{13} \mathrm{C} \mathrm{NMR}\left(50 \mathrm{MHz}, \mathrm{CDCl}_{3}\right)$

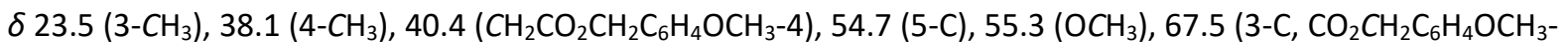
4), 114.1, 124.1, 127.5, 130.3, 147.8, 148.0, 160.0 (aromatic C), $167.6\left(\mathrm{CO}_{2} \mathrm{CH}_{2} \mathrm{C}_{6} \mathrm{H}_{4} \mathrm{OCH}_{3}-4\right), 169.4,172.5$ (2,6C); HRMS (ESI): $[\mathrm{M}+\mathrm{Na}]^{+}$calcd for $\mathrm{C}_{22} \mathrm{H}_{23} \mathrm{~N}_{3} \mathrm{O}_{7}, 464.1434$, found 464.1424 .

\subsubsection{3,4-Dimethyl-2,6-dioxo-3-phenyl-1-piperazineacetic acid $\mathbf{5 7}$}

Prepared from benzyl ester $\mathbf{5 2}(1.3 \mathrm{~g}, 3.55 \mathrm{mmol})$ by catalytic hydrogenolysis $\left(\mathrm{H}_{2} / 10 \% \mathrm{Pd}-\mathrm{C}, 156 \mathrm{mg}\right)$ in abs EtOH $(90 \mathrm{~mL})$ as described for compound 33. The obtained white foamy solid strongly binds the hydrogenation solvent. Removal of the entrapped solvent as in $\mathbf{3 3}$ gave the title compound $\mathbf{5 7}$ as a glass solid $(973 \mathbf{m g}$, almost quantitative yield): ${ }^{1} \mathrm{H} \mathrm{NMR}\left(400 \mathrm{MHz}, \mathrm{CDCl}_{3}\right) \delta 1.67\left(\mathrm{~s}, 3 \mathrm{H}, 3-\mathrm{CH}_{3}\right), 2.57\left(\mathrm{~s}, 3 \mathrm{H}, 4-\mathrm{CH}_{3}\right), 3.40-3.73(\mathrm{q}, \mathrm{AB}, 2 \mathrm{H}$, $J_{A B}=18.0 \mathrm{~Hz}, 5-\mathrm{H}$ ), 4.58-4.73 (q, $\mathrm{AB}, 2 \mathrm{H}, J_{A B} \simeq 17.0 \mathrm{~Hz}, \mathrm{CH}_{2} \mathrm{CO}_{2} \mathrm{H}$ ), 7.27-7.45 (m, 5H, aromatic H), 9.30-10.10 (br s, $\left.1 \mathrm{H}, \mathrm{CO}_{2} \mathrm{H}\right) ;{ }^{13} \mathrm{C} \mathrm{NMR}\left(100 \mathrm{MHz}, \mathrm{CDCl}_{3}\right) \delta 24.3\left(3-\mathrm{CH}_{3}\right), 38.2\left(4-\mathrm{CH}_{3}\right), 40.1\left(\mathrm{CH}_{2} \mathrm{CO}_{2} \mathrm{H}\right), 55.0(5-\mathrm{C}), 68.1(3-\mathrm{C}), 126.1$, 128.4, 129.0 (2,3,4,5,6-aromatic C), 140.4 (1-aromatic C), $170.3\left(\mathrm{CO}_{2} \mathrm{H}\right), 173.3,173.6$ (2,6-C); ESI ${ }^{+} \mathrm{MS}: \mathrm{m} / \mathrm{z} 277.1$ $[\mathrm{M}+\mathrm{H}]^{+}$.

4.1.32. 4-Methyl-2,6-Dioxo-3-phenyl-3-propyl-1-piperazineacetic acid $\mathbf{5 8}$ 
Prepared from benzyl ester $\mathbf{5 3}(2.6 \mathrm{~g}, 6.59 \mathrm{mmol})$ by catalytic hydrogenolysis $\left(\mathrm{H}_{2} / 10 \% \mathrm{Pd}-\mathrm{C}, 312 \mathrm{mg}\right)$ in abs EtOH $(165 \mathrm{~mL})$ as described for compound 33. The obtained white foamy solid strongly binds the hydrogenation solvent. Removal of the entrapped solvent as in $\mathbf{3 3}$ gave the title compound $\mathbf{5 8}$ as an off-white gum (1.98 $\mathbf{~ g}$, $99 \%)$. The hydrochloride salt $(\mathbf{5 8} \cdot \mathbf{H C l}$ ) was prepared by the same way described for $\mathbf{3 3} \cdot \mathbf{H C l}$, and obtained as a white solid (decomposed gradually above $85^{\circ} \mathrm{C}$ ): ${ }^{1} \mathrm{H} \mathrm{NMR}\left(400 \mathrm{MHz}\right.$, DMSO- $\left.d_{6}\right) \delta 0.81\left(\mathrm{t}, 3 \mathrm{H}, \mathrm{J}=7.3 \mathrm{~Hz}, \mathrm{CH}_{3}\left(\mathrm{CH}_{2}\right)_{2}\right)$, 1.03-1.28 (dm, $2 \mathrm{H}, \mathrm{CH}_{3} \mathrm{CH}_{2} \mathrm{CH}_{2}$ ), 1.82-1.93 (m, $\left.1 \mathrm{H}, \mathrm{CH}_{3} \mathrm{CH}_{2} \mathrm{CHH}\right), 2.08-2.21\left(\mathrm{~m}, 1 \mathrm{H}, \mathrm{CH}_{3} \mathrm{CH}_{2} \mathrm{CHH}\right), 2.38(\mathrm{~s}, 3 \mathrm{H}$, $\left.{ }^{+} \mathrm{NHCH}_{3}\right), 3.32-3.61\left(\mathrm{q}, \mathrm{AB}, 2 \mathrm{H}, \mathrm{J}_{\mathrm{AB}}=18.2 \mathrm{~Hz}, 5-\mathrm{H}\right), 3.66-4.35\left(\mathrm{br} \mathrm{s}, 2 \mathrm{H},{ }^{+} \mathrm{NHCH}_{3}, \mathrm{CO}_{2} \mathrm{H}\right), 4.41\left(\mathrm{~s}, 2 \mathrm{H}, \mathrm{CH}_{2} \mathrm{CO}_{2} \mathrm{H}\right), 7.30-$ $7.43(\mathrm{~m}, 5 \mathrm{H}$, aromatic $\mathrm{H}) ;{ }^{13} \mathrm{CNMR}\left(50 \mathrm{MHz}\right.$, DMSO-d $\left.d_{6}\right) \delta 14.1\left(\mathrm{CH}_{3}\left(\mathrm{CH}_{2}\right)_{2}\right), 16.4\left(\mathrm{CH}_{3} \mathrm{CH}_{2} \mathrm{CH}_{2}\right), 36.9\left({ }^{+} \mathrm{NHCH}_{3}\right), 37.5$ $\left(\mathrm{CH}_{3} \mathrm{CH}_{2} \mathrm{CH}_{2}\right), 40.3\left(\mathrm{CH}_{2} \mathrm{CO}_{2} \mathrm{H}\right), 53.8(5-\mathrm{C}), 69.9$ (3-C), 127.1, 128.1, 128.4 (2,3,4,5,6-aromatic C), 136.0 (1-aromatic C), $168.9\left(\mathrm{CO}_{2} \mathrm{H}\right), 169.3,173.0$ (2,6-C). Anal. Calcd for $\mathrm{C}_{16} \mathrm{H}_{21} \mathrm{ClN}_{2} \mathrm{O}_{4}$ : C, 56.39; $\mathrm{H}, 6.21 ; \mathrm{N}, 8.22$; Found: C, 56.02; $\mathrm{H}, 5.99 ; \mathrm{N}, 8.53$.

\subsubsection{3-Butyl-4-methyl-2,6-dioxo-3-phenyl-1-piperazineacetic acid $\mathbf{5 9}$}

Benzyl ester 54 (1.92 g, $4.7 \mathrm{mmol}$ ) was subjected to catalytic hydrogenolysis $\left(\mathrm{H}_{2} / 10 \% \mathrm{Pd}-\mathrm{C}, 230 \mathrm{mg}\right)$ in abs EtOH $\left(118 \mathrm{~mL}\right.$ ) as described in $\mathbf{3 3}$ to afford the title compound $\mathbf{5 9}$ as a glass solid $(1.48 \mathrm{~g}, 99 \%):{ }^{1} \mathrm{H}$ NMR $(600 \mathrm{MHz}$, $\left.\mathrm{CDCl}_{3}\right) \delta 0.83\left(\mathrm{t}, 3 \mathrm{H}, J \simeq 7.0 \mathrm{~Hz}, \mathrm{CH}_{3}\left(\mathrm{CH}_{2}\right)_{3}\right), 1.06-1.14\left(\mathrm{~m}, 1 \mathrm{H}, \mathrm{CH}_{3} \mathrm{CH}_{2} \mathrm{CHHCH}_{2}\right), 1.21-1.32\left(\mathrm{~m}, 3 \mathrm{H}, \mathrm{CH}_{3} \mathrm{CH}_{2} \mathrm{CHHCH}_{2}\right)$, 2.08-2.27 (dm, $\left.2 \mathrm{H}, \mathrm{CH}_{3}\left(\mathrm{CH}_{2}\right)_{2} \mathrm{CH}_{2}\right), 2.77\left(\mathrm{~s}, 3 \mathrm{H}, 4-\mathrm{CH}_{3}\right), 3.70-3.89$ (q, $\left.\mathrm{AB}, 2 \mathrm{H}, J_{A B}=17.9 \mathrm{~Hz}, 5-\mathrm{H}\right), 4.66(\mathrm{~s}, 2 \mathrm{H}$, $\mathrm{CH}_{2} \mathrm{CO}_{2} \mathrm{H}$ ), 5.20-7.20 ( $\mathrm{v}$ br s, $\left.1 \mathrm{H}, \mathrm{CO}_{2} \mathrm{H}\right), 7.33-7.53(\mathrm{~m}, 5 \mathrm{H}$, aromatic $\mathrm{H}) ;{ }^{13} \mathrm{C} \mathrm{NMR}\left(50 \mathrm{MHz}, \mathrm{CDCl}_{3}\right) \delta 13.9$ $\left(\mathrm{CH}_{3}\left(\mathrm{CH}_{2}\right)_{3}\right), 23.0\left(\mathrm{CH}_{3} \mathrm{CH}_{2}\left(\mathrm{CH}_{2}\right)_{2}\right), 25.6\left(\mathrm{CH}_{3} \mathrm{CH}_{2} \mathrm{CH}_{2} \mathrm{CH}_{2}\right), 35.9\left(\mathrm{CH}_{3}\left(\mathrm{CH}_{2}\right)_{2} \mathrm{CH}_{2}\right), 38.3\left(4-\mathrm{CH}_{3}\right), 40.6\left(\mathrm{CH}_{2} \mathrm{CO}_{2} \mathrm{H}\right), 54.2$ (5-C), 72.1 (3-C), 127.2, 129.3 (2,3,4,5,6-aromatic C), 134.4 (1-aromatic C), $167.4\left(\mathrm{CO}_{2} \mathrm{H}\right), 171.1,171.6(2,6-\mathrm{C})$; $\mathrm{ESI}^{+} \mathrm{MS}: \mathrm{m} / \mathrm{z} 319.2[\mathrm{M}+\mathrm{H}]^{+}$.

4.1.34. 3-(4-Fluorophenyl)-3,4-dimethyl-2,6-dioxo-1-piperazineacetic acid 60

Prepared from benzyl ester $55(1.75 \mathrm{~g}, 4.55 \mathrm{mmol})$ by catalytic hydrogenolysis $\left(\mathrm{H}_{2} / 10 \% \mathrm{Pd}-\mathrm{C}, 210 \mathrm{mg}\right)$ in abs $\mathrm{EtOH}(114 \mathrm{~mL})$ as described for compound 33 . The obtained white foamy solid strongly binds the hydrogenation solvent. Removal of the entrapped solvent as in $\mathbf{3 3}$ gave the title compound $\mathbf{6 0}$ as a glass solid $(1.33 \mathrm{~g}$, almost quantitative yield): ${ }^{1} \mathrm{H} N M R\left(400 \mathrm{MHz}, \mathrm{CDCl}_{3}\right) \delta 1.66\left(\mathrm{~s}, 3 \mathrm{H}, 3-\mathrm{CH}_{3}\right), 2.54\left(\mathrm{~s}, 3 \mathrm{H}, 4-\mathrm{CH}_{3}\right), 3.44-3.70(\mathrm{q}, \mathrm{AB}, 2 \mathrm{H}$, $\left.J_{A B}=18.1 \mathrm{~Hz}, 5-\mathrm{H}\right), 4.58-4.73\left(\mathrm{q}, \mathrm{AB}, 2 \mathrm{H}, \mathrm{J}_{A B} \simeq 17.1 \mathrm{~Hz}, \mathrm{CH}_{2} \mathrm{CO}_{2} \mathrm{H}\right), 7.05(\mathrm{t}, 2 \mathrm{H}, \mathrm{J} \simeq 8.6 \mathrm{~Hz}, 3,5$-aromatic H), 7.38-7.48 (q, $2 \mathrm{H}, J=5.2 \mathrm{~Hz}, 2,6$-aromatic $\mathrm{H}), 9.98-10.22\left(\mathrm{br} \mathrm{s}, 1 \mathrm{H}, \mathrm{CO}_{2} \mathrm{H}\right) ;{ }^{13} \mathrm{C} \mathrm{NMR}\left(50 \mathrm{MHz}, \mathrm{CDCl}_{3}\right) \delta 23.7\left(3-\mathrm{CH}_{3}\right), 38.1(4-$ $\left.\mathrm{CH}_{3}\right), 40.1\left(\mathrm{CH}_{2} \mathrm{CO}_{2} \mathrm{H}\right), 54.7(5-\mathrm{C}), 67.5(3-\mathrm{C}), 115.6,116.0$ (d, $J_{C-F}=21.3 \mathrm{~Hz}, 3,5$-aromatic C), 128.0, 128.2 (d, $J_{C-}$ ${ }_{F}=7.9 \mathrm{~Hz}, 2$,6-aromatic C), 136.1 (1-aromatic C), 160.0, 164.9 (d, $J_{C-F}=246 \mathrm{~Hz}$, 4-aromatic C), $170.1\left(\mathrm{CO}_{2} \mathrm{H}\right), 172.8$, 173.4 (2,6-C); ESI+ MS: m/z $295.3[\mathrm{M}+\mathrm{H}]^{+}$.

\subsubsection{2-Phenyl-2-propylaminopropanamide 66}

Acetophenone 18 (3.5 g, $29 \mathrm{mmol})$ was subjected to the Strecker reaction with $n$-propylamine hydrochloride $(2.87 \mathrm{~g}, 30 \mathrm{mmol})$ and sodium cyanide $(1.47 \mathrm{~g}, 30 \mathrm{mmol})$ following the procedure described for the preparation of compound 23. The obtained crude oily $\alpha$-aminonitrile was then hydrated with $\mathrm{H}_{2} \mathrm{SO}_{4} 97 \%$ ( $37 \mathrm{~mL}$ ) as described in 23. The resulting crude thick oil was purified by column chromatography eluting with AcOEt- $n$-hexane $1: 4$ to 1:0 to afford the title compound 66 as an off-yellow solid ( $2.7 \mathrm{~g}, 45 \%$ overall yield). An analytical sample was obtained as white crystals upon recrystallization of the product from $\mathrm{Et}_{2} \mathrm{O}-n$-pentane. $\mathrm{Mp} 56-57^{\circ} \mathrm{C} .{ }^{1} \mathrm{H} \mathrm{NMR}(400$ $\left.\mathrm{MHz}, \mathrm{CDCl}_{3}\right) \delta 0.91\left(\mathrm{t}, 3 \mathrm{H}, J=7.4 \mathrm{~Hz}, \mathrm{HN}\left(\mathrm{CH}_{2}\right)_{2} \mathrm{CH}_{3}\right), 1.40-1.54\left(\mathrm{~m}, 2 \mathrm{H}, \mathrm{HNCH}_{2} \mathrm{CH}_{2} \mathrm{CH}_{3}\right), 1.56-1.93$ (v br s, $1 \mathrm{H}$, $\left.\mathrm{HN}\left(\mathrm{CH}_{2}\right)_{2} \mathrm{CH}_{3}\right), 1.72\left(\mathrm{~s}, 3 \mathrm{H}, \mathrm{CH}_{3}\right), 2.32-2.52$ (sym m, 2H, $\left.\mathrm{HNCH}_{2} \mathrm{CH}_{2} \mathrm{CH}_{3}\right), 6.50$ (br s, $1 \mathrm{H}, \mathrm{CONHH}$ ), 7.01 (br s, $1 \mathrm{H}$, CONHH), 7.20-7.37 (m, 3H, aromatic $\mathrm{H}), 7.51(\mathrm{~d}, 2 \mathrm{H}, \mathrm{J}=7.2 \mathrm{~Hz}$, aromatic $\mathrm{H}) ;{ }^{13} \mathrm{C} \mathrm{NMR}\left(50 \mathrm{MHz}, \mathrm{CDCl}_{3}\right) \delta 11.8$ $\left(\mathrm{HN}\left(\mathrm{CH}_{2}\right)_{2} \mathrm{CH}_{3}\right), 22.6\left(\mathrm{CH}_{3}\right), 24.0\left(\mathrm{HNCH}_{2} \mathrm{CH}_{2} \mathrm{CH}_{3}\right), 45.3\left(\mathrm{HNCH}_{2} \mathrm{CH}_{2} \mathrm{CH}_{3}\right), 64.8\left(\mathrm{Ph}\left(\mathrm{CH}_{3}\right) \mathrm{CNH}\right), 126.2,127.4,128.5$ (2,3,4,5,6-aromatic C), 143.0 (1-aromatic C), $178.3\left(\mathrm{CONH}_{2}\right)$. Anal. Calcd for $\mathrm{C}_{12} \mathrm{H}_{18} \mathrm{~N}_{2} \mathrm{O}: \mathrm{C}, 69.87 ; \mathrm{H}, 8.80 ; \mathrm{N}$, 13.58; Found: C, 70.14; H, 9.12; N, 13.61.

\subsubsection{2-Butylamino-2-pheny/propanamide 67}

Acetophenone $18(3.5 \mathrm{~g}, 29 \mathrm{mmol})$ was subjected to the Strecker reaction with $n$-butylamine hydrochloride $(3.29 \mathrm{~g}, 30 \mathrm{mmol})$ and sodium cyanide $(1.47 \mathrm{~g}, 30 \mathrm{mmol})$ following the procedure described for the preparation 
of compound 23. The crude oily $\alpha$-aminonitrile obtained was then hydrated with $\mathrm{H}_{2} \mathrm{SO}_{4} 97 \%(37 \mathrm{~mL})$. The resulting crude thick oil was purified by column chromatography eluting with AcOEt- $n$-hexane 1:3 to 1:0 to afford the title compound 67 as a white crystalline solid ( $3 \mathrm{~g}, 47 \%$ overall yield): $\mathrm{mp} 83-85^{\circ} \mathrm{C}$ (Et ${ }_{2} \mathrm{O}-n$-pentane); ${ }^{1} \mathrm{H} \mathrm{NMR}\left(400 \mathrm{MHz}, \mathrm{CDCl}_{3}\right) \delta 0.89$ (t, $\left.3 \mathrm{H}, J=7.2 \mathrm{~Hz}, \mathrm{HN}\left(\mathrm{CH}_{2}\right)_{3} \mathrm{CH}_{3}\right), 1.10-1.62$ (v br s, $\left.1 \mathrm{H}, \mathrm{HN}\left(\mathrm{CH}_{2}\right)_{3} \mathrm{CH}_{3}\right), 1.27-1.50$ (dm, $\left.4 \mathrm{H}, \mathrm{HNCH}_{2} \mathrm{CH}_{2} \mathrm{CH}_{2} \mathrm{CH}_{3}\right), 1.72\left(\mathrm{~s}, 3 \mathrm{H}, \mathrm{CH}_{3}\right), 2.36-2.53$ (sym m, $\left.2 \mathrm{H}, \mathrm{HNCH}_{2}\left(\mathrm{CH}_{2}\right)_{2} \mathrm{CH}_{3}\right), 6.33$ (s, $1 \mathrm{H}, \mathrm{CONHH}$ ), $7.01(\mathrm{~s}, 1 \mathrm{H}, \mathrm{CONHH}), 7.20-7.38(\mathrm{~m}, 3 \mathrm{H}$, aromatic $\mathrm{H}), 7.51(\mathrm{~d}, 2 \mathrm{H}, \mathrm{J}=7.2 \mathrm{~Hz}$, aromatic $\mathrm{H}) ;{ }^{13} \mathrm{C} \mathrm{NMR}\left(50 \mathrm{MHz}, \mathrm{CDCl}_{3}\right)$ ס $14.1\left(\mathrm{HN}\left(\mathrm{CH}_{2}\right)_{3} \mathrm{CH}_{3}\right), 20.5\left(\mathrm{HN}\left(\mathrm{CH}_{2}\right)_{2} \mathrm{CH}_{2} \mathrm{CH}_{3}\right), 22.6\left(\mathrm{CH}_{3}\right), 33.1\left(\mathrm{HNCH}_{2} \mathrm{CH}_{2} \mathrm{CH}_{2} \mathrm{CH}_{3}\right), 43.2\left(\mathrm{HNCH}_{2}\left(\mathrm{CH}_{2}\right)_{2} \mathrm{CH}_{3}\right), 64.9$ $\left(\mathrm{Ph}\left(\mathrm{CH}_{3}\right) \mathrm{CNH}\right), 126.2,127.4,128.5$ (2,3,4,5,6-aromatic $\left.\mathrm{C}\right), 143.1$ (1-aromatic $\left.\mathrm{C}\right), 178.3\left(\mathrm{CONH}_{2}\right)$. Anal. Calcd for $\mathrm{C}_{13} \mathrm{H}_{20} \mathrm{~N}_{2} \mathrm{O}: \mathrm{C}, 70.87 ; \mathrm{H}, 9.15 ; \mathrm{N}, 12.72$; Found: $\mathrm{C}, 71.04 ; \mathrm{H}, 9.31 ; \mathrm{N}, 12.52$.

\subsubsection{7. $\mathrm{N}$-[(1-Aminocarbonyl-1-phenyl)ethyl]-N-propylglycine ethyl ester 68}

Sodium bicarbonate (336 mg, $4 \mathrm{mmol}$ ) and ethyl bromoacetate $(731 \mathrm{mg}, 4.38 \mathrm{mmol}$ ) were added successively to a solution of the aminoamide derivative $66(765 \mathrm{mg}, 3.71 \mathrm{mmol})$ in dry DMF (46 mL), and the resulting mixture was heated at $40-43^{\circ} \mathrm{C}$ for 6 days with stirring. The reaction mixture was then poured into ice-water ( $\left.40 \mathrm{~mL}\right)$ and extracted with ACOEt $(3 \times 40 \mathrm{~mL})$. The combined organic phase was washed once with brine $(40 \mathrm{~mL})$, dried $\left(\mathrm{Na}_{2} \mathrm{SO}_{4}\right)$ and concentrated to dryness under reduced pressure. The remaining oily residue was chromatographed on silica gel column with AcOEt- $n$-hexane 1:2, as eluent, to afford the title compound 68 as a white crystalline solid (615 mg, 57\%): $\mathrm{mp} 105-106{ }^{\circ} \mathrm{C}$ ( $\mathrm{Et}_{2} \mathrm{O}-n$-pentane); ${ }^{1} \mathrm{H} \mathrm{NMR}\left(400 \mathrm{MHz}, \mathrm{CDCl}_{3}\right) \delta 0.80(\mathrm{t}, 3 \mathrm{H}$, $\left.J \simeq 7.4 \mathrm{~Hz}, \mathrm{~N}\left(\mathrm{CH}_{2}\right)_{2} \mathrm{CH}_{3}\right), 1.26\left(\mathrm{t}, 3 \mathrm{H}, \mathrm{J}=7.2 \mathrm{~Hz}, \mathrm{CO}_{2} \mathrm{CH}_{2} \mathrm{CH}_{3}\right), 1.29-1.48\left(\mathrm{~m}, 2 \mathrm{H}, \mathrm{NCH}_{2} \mathrm{CH}_{2} \mathrm{CH}_{3}\right), 1.65\left(\mathrm{~s}, 3 \mathrm{H}, \mathrm{CH}_{3}\right), 2.23-$ $2.50\left(\mathrm{dm}, 2 \mathrm{H}, \mathrm{NCH}_{2} \mathrm{CH}_{2} \mathrm{CH}_{3}\right), 3.10-3.40$ (q, $\left.\mathrm{AB}, 2 \mathrm{H}, \mathrm{J}_{\mathrm{AB}} \simeq 17.4 \mathrm{~Hz}, \mathrm{NCH}_{2} \mathrm{CO}_{2} \mathrm{CH}_{2} \mathrm{CH}_{3}\right), 4.11-4.22$ (qd, $2 \mathrm{H}, \mathrm{J}_{1} \simeq 1.8 \mathrm{~Hz}$, $\left.J_{2} \simeq 7.0 \mathrm{~Hz}, \mathrm{CO}_{2} \mathrm{CH}_{2} \mathrm{CH}_{3}\right), 5.60(\mathrm{~s}, 1 \mathrm{H}, \mathrm{CONHH}), 7.21-7.36(\mathrm{~m}, 3 \mathrm{H}$, aromatic $\mathrm{H}), 7.63(\mathrm{~d}, 2 \mathrm{H}, J=7.6 \mathrm{~Hz}$, aromatic $\mathrm{H})$, $7.82(\mathrm{~s}, 1 \mathrm{H}, \mathrm{CONHH}) ;{ }^{13} \mathrm{C} \mathrm{NMR}\left(50 \mathrm{MHz}, \mathrm{CDCl}_{3}\right) \delta 11.8\left(\mathrm{NCH}_{2} \mathrm{CH}_{2} \mathrm{CH}_{3}\right), 14.2\left(\mathrm{CO}_{2} \mathrm{CH}_{2} \mathrm{CH}_{3}\right) 14.8\left(\mathrm{CH}_{3}\right), 22.1$ $\left(\mathrm{NCH}_{2} \mathrm{CH}_{2} \mathrm{CH}_{3}\right), 55.0\left(\mathrm{NCH}_{2} \mathrm{CO}_{2} \mathrm{CH}_{2} \mathrm{CH}_{3}\right), 55.5\left(\mathrm{NCH}_{2} \mathrm{CH}_{2} \mathrm{CH}_{3}\right), 61.2\left(\mathrm{CO}_{2} \mathrm{CH}_{2} \mathrm{CH}_{3}\right), 72.4\left(\mathrm{Ph}\left(\mathrm{CH}_{3}\right) \mathrm{CNH}\right), 127.2,127.7$, 128.5 (2,3,4,5,6-aromatic C), 141.7 (1-aromatic C), $173.5\left(\mathrm{CO}_{2} \mathrm{CH}_{2} \mathrm{CH}_{3}\right), 176.8\left(\mathrm{CONH}_{2}\right)$. Anal. Calcd for $\mathrm{C}_{16} \mathrm{H}_{24} \mathrm{~N}_{2} \mathrm{O}_{3}$ : C, 65.73; $\mathrm{H}, 8.27 ; \mathrm{N}, 9.58 ;$ Found: $\mathrm{C}, 65.92 ; \mathrm{H}, 8.65 ; \mathrm{N}, 9.54$.

4.1.38. $\mathrm{N}$-[(1-Aminocarbonyl-1-phenyl)ethyl]-N-butylglycine ethyl ester 69

Prepared from the aminoamide derivative $67(881 \mathrm{mg}, 4 \mathrm{mmol})$ by the same procedure described for compound 68. The resulting crude thick oil was chromatographed on silica gel column with AcOEt- $n$-hexane 1:3, as eluent, to afford the title compound 69 as a white solid (573 mg, 47\%): $\mathrm{mp} 108-110{ }^{\circ} \mathrm{C}$ ( $\mathrm{Et}_{2} \mathrm{O}-n$-pentane); ${ }^{1} \mathrm{H}$ NMR (400 $\left.\mathrm{MHz}, \mathrm{CDCl}_{3}\right) \delta 0.80\left(\mathrm{t}, 3 \mathrm{H}, \mathrm{J}=7.2 \mathrm{~Hz}, \mathrm{~N}\left(\mathrm{CH}_{2}\right)_{3} \mathrm{CH}_{3}\right), 1.08-1.46\left(\mathrm{~m}, 4 \mathrm{H}, \mathrm{NCH}_{2} \mathrm{CH}_{2} \mathrm{CH}_{2} \mathrm{CH}_{3}\right), 1.27(\mathrm{t}, 3 \mathrm{H}, \mathrm{J} \simeq 7.0 \mathrm{~Hz}$, $\left.\mathrm{CO}_{2} \mathrm{CH}_{2} \mathrm{CH}_{3}\right), 1.64\left(\mathrm{~s}, 3 \mathrm{H}, \mathrm{CH}_{3}\right), 2.27-2.35\left(\mathrm{~m}, 1 \mathrm{H}, \mathrm{NCHH}\left(\mathrm{CH}_{2}\right)_{2} \mathrm{CH}_{3}\right), 2.42-2.55\left(\mathrm{~m}, 1 \mathrm{H}, \mathrm{NCHH}\left(\mathrm{CH}_{2}\right)_{2} \mathrm{CH}_{3}\right), 3.08-3.39$ (q, AB, $2 \mathrm{H}, J_{A B}=17.2 \mathrm{~Hz}, \mathrm{NCH}_{2} \mathrm{CO}_{2} \mathrm{CH}_{2} \mathrm{CH}_{3}$ ), 4.08-4.26 (sym m, 2H, $\mathrm{CO}_{2} \mathrm{CH}_{2} \mathrm{CH}_{3}$ ), 5.64 (br s, $1 \mathrm{H}, \mathrm{CONHH}$ ), 7.20-7.38 $(\mathrm{m}, 3 \mathrm{H}$, aromatic $\mathrm{H}), 7.63(\mathrm{~d}, 2 \mathrm{H}, \mathrm{J}=8.0 \mathrm{~Hz}$, aromatic $\mathrm{H}), 7.82(\mathrm{~s}, 1 \mathrm{H}, \mathrm{CONHH}) ;{ }^{13} \mathrm{C} \mathrm{NMR}\left(50 \mathrm{MHz}, \mathrm{CDCl}_{3}\right) \delta 14.1$ $\left(\mathrm{N}\left(\mathrm{CH}_{2}\right)_{3} \mathrm{CH}_{3}\right), 14.2\left(\mathrm{CO}_{2} \mathrm{CH}_{2} \mathrm{CH}_{3}\right), 14.8\left(\mathrm{CH}_{3}\right), 20.5\left(\mathrm{~N}\left(\mathrm{CH}_{2}\right)_{2} \mathrm{CH}_{2} \mathrm{CH}_{3}\right), 31.2\left(\mathrm{NCH}_{2} \mathrm{CH}_{2} \mathrm{CH}_{2} \mathrm{CH}_{3}\right) 53.3\left(\mathrm{NCH}_{2}\left(\mathrm{CH}_{2}\right)_{2} \mathrm{CH}_{3}\right)$, $55.0\left(\mathrm{NCH}_{2} \mathrm{CO}_{2} \mathrm{CH}_{2} \mathrm{CH}_{3}\right), 61.1\left(\mathrm{CO}_{2} \mathrm{CH}_{2} \mathrm{CH}_{3}\right), 72.4\left(\mathrm{Ph}\left(\mathrm{CH}_{3}\right) \mathrm{CNH}\right), 127.2,127.6,128.4$ (2,3,4,5,6-aromatic C), 141.8 (1-aromatic C), $173.6\left(\mathrm{CO}_{2} \mathrm{CH}_{2} \mathrm{CH}_{3}\right), 176.9\left(\mathrm{CONH}_{2}\right)$. Anal. Calcd for $\mathrm{C}_{17} \mathrm{H}_{26} \mathrm{~N}_{2} \mathrm{O}_{3}: \mathrm{C}, 66.64 ; \mathrm{H}, 8.55 ; \mathrm{N}, 9.14$; Found: C, 66.95; H, 8.81; N, 8.92 .

\subsubsection{3-Methyl-2,6-dioxo-3-phenyl-4-propyl-1-piperazineacetic acid benzyl ester $\mathbf{7 0}$}

The amide-ester derivative $68(710 \mathrm{mg}, 2.43 \mathrm{mmol})$ was converted to the title benzyl ester $\mathbf{7 0}$ following the procedure described for the preparation of compound 28. Purification of the crude product (yellowish oil) by column chromatography on silica gel (AcOEt- $n$-hexane 1:5) gave $850 \mathrm{mg}(89 \%)$ of colourless viscous oil. ${ }^{1} \mathrm{H}$ NMR $\left(400 \mathrm{MHz}, \mathrm{CDCl}_{3}\right) \delta 0.82\left(\mathrm{t}, 3 \mathrm{H}, \mathrm{J} \simeq 7.4 \mathrm{~Hz}, \mathrm{~N}\left(\mathrm{CH}_{2}\right)_{2} \mathrm{CH}_{3}\right), 1.32-1.48\left(\mathrm{~m}, 2 \mathrm{H}, \mathrm{NCH}_{2} \mathrm{CH}_{2} \mathrm{CH}_{3}\right), 1.56\left(\mathrm{~s}, 3 \mathrm{H}, 3-\mathrm{CH}_{3}\right), 2.38-$ $2.49\left(\mathrm{~m}, 1 \mathrm{H}, \mathrm{NCHHCH}_{2} \mathrm{CH}_{3}\right), 2.55-2.65\left(\mathrm{~m}, 1 \mathrm{H}, \mathrm{NCHHCH}_{2} \mathrm{CH}_{3}\right), 3.40-3.57$ (q, $\left.\mathrm{AB}, 2 \mathrm{H}, \mathrm{J}_{A B} \simeq 18.2 \mathrm{~Hz}, 5-\mathrm{H}\right)$, 4.47-4.65 (q, $\mathrm{AB}, 2 \mathrm{H}, \mathrm{J}_{\mathrm{AB}}=16.8 \mathrm{~Hz}, \mathrm{CH}_{2} \mathrm{CO}_{2} \mathrm{CH}_{2} \mathrm{Ph}$ ), 5.10 (s, $2 \mathrm{H}, \mathrm{CO}_{2} \mathrm{CH}_{2} \mathrm{Ph}$ ), 7.15-7.40 (complex m, $10 \mathrm{H}$, aromatic H); ${ }^{13} \mathrm{C} \mathrm{NMR}$

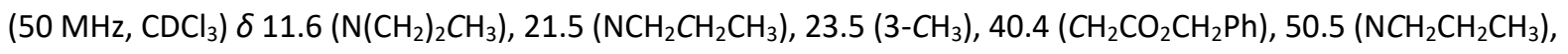
$51.1(5-\mathrm{C}), 67.4\left(\mathrm{CO}_{2} \mathrm{CH}_{2} \mathrm{Ph}\right), 68.0$ (3-C), 126.2, 128.1, 128.4, 128.6, 128.7, 128.9, 129.3, 129.4, 135.2, 141.4 
(aromatic C), $167.7\left(\mathrm{CO}_{2} \mathrm{CH}_{2} \mathrm{Ph}\right), 170.8,173.9$ (2,6-C); HRMS (ESI): [M+H] $]^{+}$calcd for $\mathrm{C}_{23} \mathrm{H}_{26} \mathrm{~N}_{2} \mathrm{O}_{4}, 395.1971$, found 395.1968.

4.1.40. 4-Butyl-3-methyl-2,6-dioxo-3-phenyl-1-piperazineacetic acid benzyl ester $\mathbf{7 1}$

The amide-ester derivative 69 (560 mg, $1.83 \mathrm{mmol}$ ) was converted to the title benzyl ester $\mathbf{7 1}$ following the procedure described for the preparation of compound 28. Purification of the crude product (off-yellow thick oil) by column chromatography on silica gel (AcOEt- $n$-hexane 1:6) gave $606 \mathrm{mg}(81 \%)$ of colourless viscous oil. ${ }^{1} \mathrm{H}$ NMR (400 MHz, $\left.\mathrm{CDCl}_{3}\right) \delta 0.90\left(\mathrm{t}, 3 \mathrm{H}, \mathrm{J}=7.2 \mathrm{~Hz}, \mathrm{~N}\left(\mathrm{CH}_{2}\right)_{3} \mathrm{CH}_{3}\right.$ ), 1.21-1.53 (complex m, 4H, $\mathrm{NCH}_{2} \mathrm{CH}_{2} \mathrm{CH}_{2} \mathrm{CH}_{3}$ ), 1.65 (s, $\left.3 \mathrm{H}, 3-\mathrm{CH}_{3}\right), 2.46-2.58\left(\mathrm{~m}, 1 \mathrm{H}, \mathrm{NCHH}\left(\mathrm{CH}_{2}\right)_{2} \mathrm{CH}_{3}\right), 2.68-2.81\left(\mathrm{~m}, 1 \mathrm{H}, \mathrm{NCHH}\left(\mathrm{CH}_{2}\right)_{2} \mathrm{CH}_{3}\right), 3.48-3.68\left(\mathrm{q}, \mathrm{AB}, 2 \mathrm{H}, \mathrm{J}_{A B}=18.0\right.$ $\mathrm{Hz}, 5-\mathrm{H}$ ), 4.53-4.76 (q, $\mathrm{AB}, 2 \mathrm{H}, \mathrm{J}_{A B}=16.8 \mathrm{~Hz}, \mathrm{CH}_{2} \mathrm{CO}_{2} \mathrm{CH}_{2} \mathrm{Ph}$ ), 5.19 (s, $2 \mathrm{H}, \mathrm{CO}_{2} \mathrm{CH}_{2} \mathrm{Ph}$ ), 7.20-7.50 (complex m, 10H,

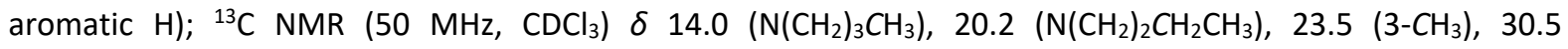
$\left(\mathrm{NCH}_{2} \mathrm{CH}_{2} \mathrm{CH}_{2} \mathrm{CH}_{3}\right), 40.5\left(\mathrm{CH}_{2} \mathrm{CO}_{2} \mathrm{CH}_{2} \mathrm{Ph}\right), 48.4\left(\mathrm{NCH}_{2}\left(\mathrm{CH}_{2}\right)_{2} \mathrm{CH}_{3}\right), 51.1$ (5-C), $67.4\left(\mathrm{CO}_{2} \mathrm{CH}_{2} \mathrm{Ph}\right), 68.0$ (3-C), 126.2, 128.2, 128.4, 128.6, 128.7, 128.9, 135.3, 141.4 (aromatic C), $167.7\left(\mathrm{CO}_{2} \mathrm{CH}_{2} \mathrm{Ph}\right), 170.8,173.9$ (2,6-C); HRMS (ESI): $[\mathrm{M}+\mathrm{H}]^{+}$calcd for $\mathrm{C}_{24} \mathrm{H}_{28} \mathrm{~N}_{2} \mathrm{O}_{4}, 409.2127$, found 409.2150 .

4.1.41. 3-Methyl-2,6-dioxo-3-phenyl-4-propyl-1-piperazineacetic acid $\mathbf{7 2}$

Benzyl ester 70 ( $1.25 \mathrm{~g}, 3.17 \mathrm{mmol})$ was subjected to catalytic hydrogenolysis $\left(\mathrm{H}_{2} / 10 \% \mathrm{Pd}-\mathrm{C}, 150 \mathrm{mg}\right)$ in abs EtOH $(143 \mathrm{~mL})$ as described in $\mathbf{3 3}$ to give a colourless glass solid. Trituration of this product material with $\mathrm{Et}_{2} \mathrm{O}$ gave the title compound 72 as a white crystalline solid $(900 \mathrm{mg}, 93 \%)$. A recrystallized sample had $\mathrm{mp} 152-154{ }^{\circ} \mathrm{C}$ (from Et ${ }_{2} \mathrm{O}$ ). ${ }^{1} \mathrm{H}$ NMR $\left(400 \mathrm{MHz}, \mathrm{CDCl}_{3}\right) \delta 0.92\left(\mathrm{t}, 3 \mathrm{H}, \mathrm{J}=7.2 \mathrm{~Hz}, \mathrm{~N}\left(\mathrm{CH}_{2}\right)_{2} \mathrm{CH}_{3}\right), 1.44-1.60$ (sym m, $2 \mathrm{H}, \mathrm{NCH}_{2} \mathrm{CH}_{2} \mathrm{CH}_{3}$ ), $1.66\left(\mathrm{~s}, 3 \mathrm{H}, 3-\mathrm{CH}_{3}\right), 2.49-2.63\left(\mathrm{~m}, 1 \mathrm{H}, \mathrm{NCHHCH}{ }_{2} \mathrm{CH}_{3}\right), 2.68-2.80\left(\mathrm{~m}, 1 \mathrm{H}, \mathrm{NCHHCH} \mathrm{CH}_{3}\right), 3.47-3.70(\mathrm{q}, \mathrm{AB}, 2 \mathrm{H}$, $J_{A B}=18.4 \mathrm{~Hz}, 5-\mathrm{H}$ ), 4.53-4.73 (q, $\mathrm{AB}, 2 \mathrm{H}, J_{A B} \simeq 17.0 \mathrm{~Hz}, \mathrm{CH}_{2} \mathrm{CO}_{2} \mathrm{H}$ ), 7.20-7.50 (complex m, 5H, aromatic H), 9.6110.53 (br s, $\left.1 \mathrm{H}, \mathrm{CO}_{2} \mathrm{H}\right) ;{ }^{13} \mathrm{C} \mathrm{NMR}\left(50 \mathrm{MHz}, \mathrm{CDCl}_{3}\right) \delta 11.6\left(\mathrm{~N}_{\left(\mathrm{CH}_{2}\right)}{ }_{2} \mathrm{CH}_{3}\right), 21.5\left(\mathrm{NCH}_{2} \mathrm{CH}_{2} \mathrm{CH}_{3}\right), 23.6\left(3-\mathrm{CH}_{3}\right), 40.1$ $\left(\mathrm{CH}_{2} \mathrm{CO}_{2} \mathrm{H}\right), 50.7\left(\mathrm{NCH}_{2} \mathrm{CH}_{2} \mathrm{CH}_{3}\right), 51.1(5-\mathrm{C}), 68.0(3-\mathrm{C}), 126.2$, 128.2, 128.9 (2,3,4,5,6-aromatic C), 141.3 (1aromatic $\mathrm{C}), 170.8\left(\mathrm{CO}_{2} \mathrm{H}\right), 173.5,173.9(2,6-\mathrm{C})$. Anal. Calcd for $\mathrm{C}_{16} \mathrm{H}_{20} \mathrm{~N}_{2} \mathrm{O}_{4}: \mathrm{C}, 63.14 ; \mathrm{H}, 6.62 ; \mathrm{N}, 9.21$; Found: $\mathrm{C}$, $63.43 ; \mathrm{H}, 6.72 ; \mathrm{N}, 9.52$.

4.1.42. 4-Butyl-3-methyl-2,6-dioxo-3-phenyl-1-piperazineacetic acid 73

Benzyl ester 71 (600 mg, $1.47 \mathrm{mmol}$ ) was subjected to catalytic hydrogenolysis $\left(\mathrm{H}_{2} / 10 \% \mathrm{Pd}-\mathrm{C}, 72 \mathrm{mg}\right.$ ) in abs EtOH $(66 \mathrm{~mL})$ as described in $\mathbf{3 3}$ to give a colourless glass solid. Trituration of this product material with a diethyl ether- $n$-pentane 1:1 mixture afforded the title compound 73 as a white crystalline solid $(460 \mathrm{mg}, 98 \%)$. A specimen was recrystallized from $\mathrm{Et}_{2} \mathrm{O}-n$-pentane to give an analytical sample which had $\mathrm{mp} 102-103{ }^{\circ} \mathrm{C} .{ }^{1} \mathrm{H} \mathrm{NMR}$ $\left(400 \mathrm{MHz}, \mathrm{CDCl}_{3}\right) \delta 0.91\left(\mathrm{t}, 3 \mathrm{H}, \mathrm{J}=7.2 \mathrm{~Hz}, \mathrm{~N}\left(\mathrm{CH}_{2}\right)_{3} \mathrm{CH}_{3}\right.$ ), 1.25-1.57 (complex m, 4H, NCH $\left.\mathrm{CH}_{2} \mathrm{CH}_{2} \mathrm{CH}_{3}\right), 1.67$ (s, 3H, 3- $\left.\mathrm{CH}_{3}\right), 2.50-2.63\left(\mathrm{~m}, 1 \mathrm{H}, \mathrm{NCHH}\left(\mathrm{CH}_{2}\right)_{2} \mathrm{CH}_{3}\right), 2.75-2.85\left(\mathrm{~m}, 1 \mathrm{H}, \mathrm{NCHH}\left(\mathrm{CH}_{2}\right)_{2} \mathrm{CH}_{3}\right), 3.51-3.68\left(\mathrm{q}, \mathrm{AB}, 2 \mathrm{H}, \mathrm{J}_{A B}=18.0 \mathrm{~Hz}\right.$, 5-H), 4.53-4.72 (q, $\left.\mathrm{AB}, 2 \mathrm{H}, \mathrm{J}_{A B} \simeq 17.0 \mathrm{~Hz}, \mathrm{CH}_{2} \mathrm{CO}_{2} \mathrm{H}\right), 7.26-7.48(\mathrm{~m}, 5 \mathrm{H}$, aromatic $\mathrm{H})$, 9.80-11.1 (v br s, $\left.1 \mathrm{H}, \mathrm{CO}_{2} \mathrm{H}\right)$;

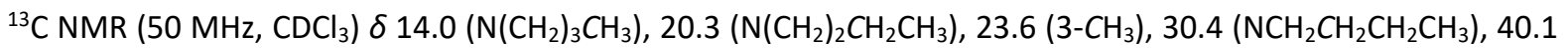
$\left(\mathrm{CH}_{2} \mathrm{CO}_{2} \mathrm{H}\right), 48.6\left(\mathrm{NCH}_{2}\left(\mathrm{CH}_{2}\right)_{2} \mathrm{CH}_{3}\right), 51.1$ (5-C), 68.1 (3-C), 126.2, 128.2, 128.9 (2,3,4,5,6-aromatic C), 141.3 (1aromatic C), $170.8\left(\mathrm{CO}_{2} \mathrm{H}\right), 173.6,173.9(2,6-\mathrm{C})$; Anal. Calcd for $\mathrm{C}_{17} \mathrm{H}_{22} \mathrm{~N}_{2} \mathrm{O}_{4}$ : C, 64.13; H, 6.97; N, 8.80; Found: C, $63.82 ; \mathrm{H}, 7.12 ; \mathrm{N}, 9.03$.

4.1.43. $\mathrm{N}$-Hydroxy-3-methyl-2,6-dioxo-3-phenyl-1-piperazineacetamide 6

1,1'-Carbonyldiimidazol (409 mg, $2.52 \mathrm{mmol}$ ) was added to a solution of carboxylic acid 33 (550 mg, $2.1 \mathrm{mmol}$ ) in dry THF (42 mL), and the mixture was allowed to stir at $28{ }^{\circ} \mathrm{C}$ for $1 \mathrm{~h}$ under argon. Then, $O$ benzylhydroxylamine hydrochloride $(402 \mathrm{mg}, 2.52 \mathrm{mmol}$ ) was added followed by triethylamine $(510 \mathrm{mg}, 5.04$ $\mathrm{mmol}$ ). After the mixture was stirred for $24 \mathrm{~h}$ at $28^{\circ} \mathrm{C}$ and $1 \mathrm{~h}$ at $45^{\circ} \mathrm{C}$ under argon, the solvent was evaporated under reduced pressure. Water $(50 \mathrm{~mL})$ was added to the residue, and the mixture was extracted with ethyl acetate $(3 \times 50 \mathrm{~mL})$. The combined organic phase was washed with brine $(2 \times 50 \mathrm{~mL})$, dried $\left(\mathrm{Na}_{2} \mathrm{SO}_{4}\right)$ and evaporated to dryness in vacuo. The viscous oily residue was purified by column chromatography on silica gel with AcOEt- $n$-hexane 2:1, as eluent, to afford the $N$-benzyloxy precursor 37 as a white foamy solid, which strongly binds the elution solvents. Removal of the entrapped solvents upon drying at $62-64{ }^{\circ} \mathrm{C}$ under high 
vacuum $\left(10^{-2} \mathrm{mmHg}\right.$ ) in an Abderhalden apparatus gave 37 as a glass solid $(572 \mathrm{mg}, 74 \%)$. This compound appears in the ${ }^{1} \mathrm{H}$ and ${ }^{13} \mathrm{C} N M R$ spectra as a mixture of $E / Z$ conformers (not assigned). ${ }^{1} \mathrm{H} \mathrm{NMR}\left(600 \mathrm{MHz}, \mathrm{CDCl}_{3}\right.$, $283 \mathrm{~K}$ ) $\delta 1.59,1.63\left(\mathrm{~s}+\mathrm{s}, 3 \mathrm{H}, \mathrm{CH}_{3}\right), 2.26-2.74(\mathrm{br} \mathrm{s}, 1 \mathrm{H}, 4-\mathrm{H}), 3.36-3.50$ (q, $\left.1 \mathrm{H}, J=18.5 \mathrm{~Hz}, 5-\mathrm{H}\right), 3.59-3.71$ (q, $1 \mathrm{H}$, $J=18.5 \mathrm{~Hz}, 5-\mathrm{H}$ ), 4.30-4.43 (q, AB, 1.14H, $\left.\mathrm{J}_{A B} \simeq 15.1 \mathrm{~Hz}, \mathrm{CH}_{2} \mathrm{CONHOCH}_{2} \mathrm{Ph}\right), 4.70$ (s, 0.94H, $\mathrm{CH}_{2} \mathrm{CONHOCH}_{2} \mathrm{Ph}$ ), 4.87, $4.91\left(\mathrm{~s}+\mathrm{s}, 2 \mathrm{H}, \mathrm{CONHOCH}_{2} \mathrm{Ph}\right.$ ), 7.28-7.50 (complex m, 10H, aromatic $\mathrm{H}$ ), $8.59\left(\mathrm{~s}, 0.48 \mathrm{H}, \mathrm{CONHOCH}_{2} \mathrm{Ph}\right.$ ), 9.37 (s, $0.57 \mathrm{H}, \mathrm{CONHOCH} 2 \mathrm{Ph}) ;{ }^{13} \mathrm{C} \mathrm{NMR}\left(150 \mathrm{MHz}, \mathrm{CDCl}_{3}, 283 \mathrm{~K}\right) \delta 28.7\left(\mathrm{CH}_{3}\right), 39.4\left(\mathrm{CH}_{2} \mathrm{CONHOCH}_{2} \mathrm{Ph}\right), 45.9(5-\mathrm{C}), 62.5$ (3-C), 78.2, 79.5 ( $\left.\mathrm{CONHOCH}_{2} \mathrm{Ph}\right), 125.6,125.7,128.1,128.5,128.7,128.8,128.9,129.1,129.3,129.4,134.0$, 134.9, 138.7 (aromatic C), 165.2, 170.3 ( $\left.\mathrm{CONHOCH}_{2} \mathrm{Ph}\right), 171.8,173.8$ (2,6-C); ESI ${ }^{+} \mathrm{MS}: \mathrm{m} / \mathrm{z} 368.2[\mathrm{M}+\mathrm{H}]^{+}$.

A solution of $O$-benzyl hydroxamate $37(570 \mathrm{mg}, 1.55 \mathrm{mmol})$ in abs $\mathrm{EtOH}(70 \mathrm{~mL})$ was hydrogenated over $10 \%$ $\mathrm{Pd}-\mathrm{C}(68 \mathrm{mg})$ at room temperature under $50 \mathrm{psi}$ of hydrogen. After $3 \mathrm{~h}$ the catalyst was filtered off, washed with $\mathrm{EtOH}(3 \times 15 \mathrm{~mL})$, and the combined filtrates were evaporated to dryness under reduced pressure. The residual material (off-white foamy solid) was chromatographed on silica gel column with AcOEt-MeOH 15:1, as eluent, to afford the title compound $\mathbf{6}$ as a white foamy solid, which strongly binds the aforementioned solvents. Removal of the entrapped solvents upon drying at $62-64{ }^{\circ} \mathrm{C}$ under high vacuum $\left(10^{-2} \mathrm{mmHg}\right)$ in an Abderhalden apparatus gave 6 as a slightly off-yellow crystalline solid (350 mg, 81\%): $\mathrm{mp} 80-83{ }^{\circ} \mathrm{C}$ (dec); ${ }^{1} \mathrm{H}$ NMR $(400 \mathrm{MHz}$, DMSO-d $\left.)_{6}\right) \delta 1.51\left(\mathrm{~s}, 3 \mathrm{H}, \mathrm{CH}_{3}\right), 3.07-3.19(\mathrm{q}, 1 \mathrm{H}, J=10.7 \mathrm{~Hz}, 5-\mathrm{H}), 3.53-3.64(\mathrm{dd}, 1 \mathrm{H}, J=4.1,18.3 \mathrm{~Hz}, 5-\mathrm{H}), 3.79-3.90$ (dd, $1 \mathrm{H}, \mathrm{J}=4.0,10.6 \mathrm{~Hz}, 4-\mathrm{H}$ ), 4.19-4.32 (q, AB, 1.5H, $\mathrm{J}_{A B}=15.6 \mathrm{~Hz}, \mathrm{CH}_{2} \mathrm{CONHOH}, E$-isomer), 4.49-4.61 (q, AB, 0.46H, $J_{A B} \approx 17.4 \mathrm{~Hz}, \mathrm{CH}_{2} \mathrm{CONHOH}, Z$-isomer), 7.27-7.52 (complex m, 5H, aromatic $\mathrm{H}$ ), 8.93 (s, 0.74H, CONHOH, E-isomer), 9.34 (s, 0.2H, CONHOH, Z-isomer), 10.21 (s, 0.2H, CONHOH, Z-isomer), 10.65 (s, 0.75H, CONHOH, E-isomer); ${ }^{13} \mathrm{C}$ NMR (100 MHz, DMSO- $\left.d_{6}\right) \delta 28.2\left(\mathrm{CH}_{3}\right), 39.0\left(\mathrm{CH}_{2} \mathrm{CONHOH}, E\right.$-isomer), $39.4\left(\mathrm{CH}_{2} \mathrm{CONHOH}, Z\right.$-isomer), $45.8(5-\mathrm{C})$, 62.1 (3-C), 126.0, 126.4, 126.6, 127.7, 128.1, 128.5, 128.6 (2,3,4,5,6-aromatic C), 140.2, 140.3 (1-aromatic C), 163.9 (CONHOH, E-isomer), 169.3 (CONHOH, Z-isomer), 171.5, 173.7, 173.8 (2,6-C); HRMS (APCl ${ }^{+}$): $[\mathrm{M}+\mathrm{H}]^{+}$calcd for $\mathrm{C}_{13} \mathrm{H}_{15} \mathrm{~N}_{3} \mathrm{O}_{4}, 278.1135$, found 278.1136 . The hydrochloride salt $(6 \cdot \mathrm{HCl})$ was prepared by treating a solution of 6 in $\mathrm{AcOEt}-\mathrm{Et}_{2} \mathrm{O}$ 2:3 with saturated solution of $\mathrm{HCl}$ in $\mathrm{Et}_{2} \mathrm{O}$ under ice cooling. The solvents were then evaporated under reduced pressure, and the white solid was triturated with $\mathrm{Et}_{2} \mathrm{O}$, filtered and dried in vacuo. $\mathrm{Mp} \mathrm{141-145}$ ${ }^{\circ} \mathrm{C}$ (dec) (slightly hygroscopic). Anal. Calcd for $\mathrm{C}_{13} \mathrm{H}_{16} \mathrm{ClN}_{3} \mathrm{O}_{4}: \mathrm{C}, 49.77 ; \mathrm{H}, 5.14 ; \mathrm{N}, 13.39$; Found: $\mathrm{C}, 49.38 ; \mathrm{H}, 5.52$; $\mathrm{N}, 13.03$.

\subsubsection{N-Hydroxy-2,6-dioxo-3-phenyl-3-propyl-1-piperazineacetamide 7}

The $\mathrm{N}$-benzyloxy precursor 38 was prepared from carboxylic acid $34(700 \mathrm{mg}, 2.41 \mathrm{mmol}$ ) following the procedure described for the preparation of compound 37 (precursor for 6 ). The crude viscous oil was purified by column chromatography on silica gel with AcOEt- $n$-hexane 2:3, as eluent, to afford $\mathbf{3 8}$ as a glass solid (675 $\mathrm{mg}, 71 \%$ ). This compound appears in the ${ }^{1} \mathrm{H}$ and ${ }^{13} \mathrm{C} \mathrm{NMR} \mathrm{spectra} \mathrm{as} \mathrm{a} \mathrm{mixture} \mathrm{of} E / Z$ conformers (not assigned). ${ }^{1} \mathrm{H} \mathrm{NMR}\left(400 \mathrm{MHz}, \mathrm{CDCl}_{3}\right) \delta 0.75\left(\mathrm{t}, 3 \mathrm{H}, \mathrm{J}=7.2 \mathrm{~Hz}, \mathrm{CH}_{3}\left(\mathrm{CH}_{2}\right)_{2}\right), 1.10-1.28\left(\mathrm{~m}, 2 \mathrm{H}, \mathrm{CH}_{3} \mathrm{CH}_{2} \mathrm{CH}_{2}\right), 1.72-2.02(\mathrm{dm}, 2 \mathrm{H}$, $\left.\mathrm{CH}_{3} \mathrm{CH}_{2} \mathrm{CH}_{2}\right), 2.43(\mathrm{~s}, 1 \mathrm{H}, 4-\mathrm{H}), 3.28-3.69(\mathrm{dm}, 2 \mathrm{H}, 5-\mathrm{H}), 4.14-4.39\left(\mathrm{~m}, 1 \mathrm{H}, \mathrm{CH}_{2} \mathrm{CONHOCH}_{2} \mathrm{Ph}\right), 4.60,4.65(\mathrm{~s}+\mathrm{s}$, $\left.0.95 \mathrm{H}, \mathrm{CH}_{2} \mathrm{CONHOCH}_{2} \mathrm{Ph}\right), 4.78\left(\mathrm{~s}, 2 \mathrm{H}, \mathrm{CONHOCH}_{2} \mathrm{Ph}\right), 7.10-7.45(\mathrm{~m}, 10 \mathrm{H}$, aromatic $\mathrm{H}), 8.71(\mathrm{~s}, 0.3 \mathrm{H}$, $\mathrm{CONHOCH} 2 \mathrm{Ph}$ ), $9.46\left(\mathrm{~s}, 0.4 \mathrm{H}, \mathrm{CONHOCH}_{2} \mathrm{Ph}\right) ;{ }^{13} \mathrm{C} \mathrm{NMR}(50 \mathrm{MHz}, \mathrm{CDCl}) \delta 14.3\left(\mathrm{CH}_{3}\left(\mathrm{CH}_{2}\right)_{2}\right), 17.2\left(\mathrm{CH}_{3} \mathrm{CH}_{2} \mathrm{CH}_{2}\right)$, $39.5\left(\mathrm{CH}_{2} \mathrm{CONHOCH}_{2} \mathrm{Ph}\right), 43.9\left(\mathrm{CH}_{3} \mathrm{CH}_{2} \mathrm{CH}_{2}\right), 45.9(5-\mathrm{C}), 65.7$ (3-C), 78.6, $79.5\left(\mathrm{CONHOCH}_{2} \mathrm{Ph}\right), 126.2,128.1,128.7$, 128.8, 129.3, 135.1, 137.8 (aromatic C), 165.4, 170.5 ( $\left.\mathrm{CONHOCH}_{2} \mathrm{Ph}\right), 171.8,173.4$ (2,6-C); El MS: m/z 396.2 $\left([\mathrm{M}+\mathrm{H}]^{+}, 9\right), 395.1\left([\mathrm{M}]^{+}, 27\right), 352.1\left(\left[\mathrm{M}-\mathrm{CH}_{2} \mathrm{CH}_{2} \mathrm{CH}_{3}\right]^{+}, 25\right), 305.1\left(\left[\mathrm{M}+\mathrm{H}-\mathrm{CH}_{2} \mathrm{Ph}\right]^{+}, 11\right), 304.0\left(\left[\mathrm{M}-\mathrm{CH}_{2} \mathrm{Ph}\right]^{+}, 67\right)$, $217.0(73), 160.0(83), 90.9(100)$.

Compound 38 (1.2 g, $3.03 \mathrm{mmol}$ ) was subjected to catalytic hydrogenation ( $\left.\mathrm{H}_{2} / 10 \% \mathrm{Pd}-\mathrm{C}, 144 \mathrm{mg}\right)$, in abs EtOH $(136 \mathrm{~mL})$ as described for the preparation of compound 6 from 37. The hydrogenation product (off-white foamy solid) was chromatographed on silica gel column eluting first with AcOEt- $n$-hexane 1:2 and then AcOEt to afford the title compound $\mathbf{7}$ as a white foamy solid. This material gave white crystals upon dissolving in $\mathrm{Et}_{2} \mathrm{O}(15 \mathrm{~mL})$ and subsequent evaporation of the solvent twice. (700 mg, 76\%): $\mathrm{mp} 148-150{ }^{\circ} \mathrm{C}$ (dec) (MeOH-Et $\left.2 \mathrm{O}\right) ;{ }^{1} \mathrm{H} \mathrm{NMR}$ (400 MHz, DMSO- $\left.d_{6}\right) \delta 0.78\left(\mathrm{t}, 3 \mathrm{H}, J=6.3,7.4 \mathrm{~Hz}, \mathrm{CH}_{3}\left(\mathrm{CH}_{2}\right)_{2}\right), 1.12-1.36\left(\mathrm{~m}, 2 \mathrm{H}, \mathrm{CH}_{3} \mathrm{CH}_{2} \mathrm{CH}_{2}\right), 1.70-1.81$ (td, $1 \mathrm{H}$, 
$J \approx 4.5 \mathrm{~Hz}, J=11.9,13.4 \mathrm{~Hz}, \mathrm{CH}_{3} \mathrm{CH}_{2} \mathrm{CHH}$ ), 1.82-1.93 (td, $1 \mathrm{H}, J=2.6-4.0 \mathrm{~Hz}, J=11.9,13.1 \mathrm{~Hz}, \mathrm{CH}_{3} \mathrm{CH}_{2} \mathrm{CHH}_{\text {), 3.16-3.27 }}$ (q, $1 \mathrm{H}, J=10.1 \mathrm{~Hz}, 5-\mathrm{H}), 3.56-3.66(\mathrm{dd}, 1 \mathrm{H}, \mathrm{J}=3.7,18.1 \mathrm{~Hz}, 5-\mathrm{H}), 3.69-3.78(\mathrm{dd}, 1 \mathrm{H}, \mathrm{J} \simeq 2.8,9.8 \mathrm{~Hz}, 4-\mathrm{H}), 4.15-4.30$ (q, $\mathrm{AB}, 1.53 \mathrm{H}, J_{A B}=15.5 \mathrm{~Hz}, \mathrm{CH}_{2} \mathrm{CONHOH}, E$-isomer), $4.45-4.59$ (q, $\mathrm{AB}, 0.41 \mathrm{H}, J_{A B}=16.8 \mathrm{~Hz}, \mathrm{CH}_{2} \mathrm{CONHOH}, \mathrm{Z}$-isomer), 7.25-7.51 (m, 5H, aromatic H), $8.92(\mathrm{~s}, 0.7 \mathrm{H}, \mathrm{CONHOH}, \mathrm{E}$-isomer), 9.33 (s, 0.2H, CONHOH, Z-isomer), 10.20 (s, $0.2 \mathrm{H}, \mathrm{CONHOH}, \mathrm{Z}$-isomer), $10.63\left(\mathrm{~s}, 0.7 \mathrm{H}, \mathrm{CONHOH}, E\right.$-isomer); ${ }^{13} \mathrm{C} \mathrm{NMR}\left(50 \mathrm{MHz}, \mathrm{DMSO}-d_{6}\right) \delta 14.2\left(\mathrm{CH}_{3}\left(\mathrm{CH}_{2}\right)_{2}\right)$, $16.7\left(\mathrm{CH}_{3} \mathrm{CH}_{2} \mathrm{CH}_{2}\right), 38.9\left(\mathrm{CH}_{2} \mathrm{CONHOH}\right), 43.4\left(\mathrm{CH}_{3} \mathrm{CH}_{2} \mathrm{CH}_{2}\right), 45.7$ (5-C), 65.0 (3-C), 126.2, 127.6, 128.5 (2,3,4,5,6aromatic C), 138.7 (1-aromatic C), 163.8 (CONHOH, E-isomer), 169.2 (CONHOH, Z-isomer), 171.3, 173.1 (2,6-C); ESI ${ }^{+}$MS: $\mathrm{m} / \mathrm{z} 306.5$ [M+H]. Anal. Calcd for $\mathrm{C}_{15} \mathrm{H}_{19} \mathrm{~N}_{3} \mathrm{O}_{4}$ : C, 59.01; $\mathrm{H}, 6.27 ; \mathrm{N}, 13.76$; Found: $\mathrm{C}, 58.87 ; \mathrm{H}, 6.30 ; \mathrm{N}$,

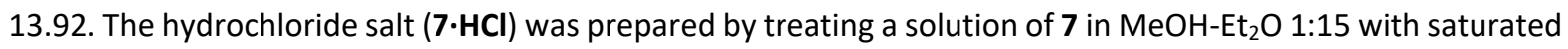
solution of $\mathrm{HCl}$ in $\mathrm{Et}_{2} \mathrm{O}$ under ice cooling, and was fully precipitated by adding $\mathrm{Et}_{2} \mathrm{O}$. The white solid was collected by vacuum filtration, triturated with $\mathrm{Et}_{2} \mathrm{O}$, and dried in vacuo (decomposed gradually above $115^{\circ} \mathrm{C}$ ). Anal. Calcd for $\mathrm{C}_{15} \mathrm{H}_{20} \mathrm{ClN}_{3} \mathrm{O}_{4} \cdot 0.15 \mathrm{Et}_{2} \mathrm{O}: \mathrm{C}, 53.09 ; \mathrm{H}, 6.14 ; \mathrm{N}, 11.90$; Found: $\mathrm{C}, 52.80 ; \mathrm{H}, 6.21 ; \mathrm{N}, 11.62$.

4.1.45. 3-Butyl-N-hydroxy-2,6-dioxo-3-phenyl-1-piperazineacetamide 8

The $N$-benzyloxy precursor 39 was prepared from carboxylic acid 35 ( $1 \mathrm{~g}, 3.29 \mathrm{mmol})$ following the procedure described for the preparation of compound $\mathbf{3 7}$ (precursor for 6). The crude oil was purified by flash column chromatography eluting with AcOEt- $n$-hexane 1:1 to afford 39 as an off-yellow glass oil (870 mg, $64 \%$ ). This compound appears in the ${ }^{1} \mathrm{H}$ and ${ }^{13} \mathrm{C} N M R$ spectra as a mixture of $E / Z$ conformers (not assigned). ${ }^{1} \mathrm{H} N M R(600$ $\left.\mathrm{MHz}, \mathrm{CDCl}_{3}\right) \delta 0.77\left(\mathrm{t}, 3 \mathrm{H}, J=5.6 \mathrm{~Hz}, \mathrm{CH}_{3}\left(\mathrm{CH}_{2}\right)_{3}\right), 1.06-1.30\left(\mathrm{~m}, 4 \mathrm{H}, \mathrm{CH}_{3} \mathrm{CH}_{2} \mathrm{CH}_{2} \mathrm{CH}_{2}\right), 1.75-2.06(\mathrm{dm}, 2 \mathrm{H}$, $\left.\mathrm{CH}_{3}\left(\mathrm{CH}_{2}\right)_{2} \mathrm{CH}_{2}\right), 2.27-2.49$ (br s, $\left.1 \mathrm{H}, 4-\mathrm{H}\right), 3.44$ (d, $\left.1 \mathrm{H}, J=17.0 \mathrm{~Hz}, 5-\mathrm{H}\right), 3.62$ (d, $\left.1 \mathrm{H}, \mathrm{J}=18.2 \mathrm{~Hz}, 5-\mathrm{H}\right), 4.28(\mathrm{br} d$, $\left.0.76 \mathrm{H}, \mathrm{J}=26.8 \mathrm{~Hz}, \mathrm{CH}_{2} \mathrm{CONHOCH}_{2} \mathrm{Ph}\right), 4.63,4.68\left(\mathrm{~s}+\mathrm{s}, 1.47 \mathrm{H}, \mathrm{CH}_{2} \mathrm{CONHOCH}_{2} \mathrm{Ph}\right), 4.83(\mathrm{~s}, 2 \mathrm{H}, \mathrm{CONHOCH} \mathrm{Ph}$ ), 7.16-7.44 (complex m, 10H, aromatic $\mathrm{H}$ ), 8.32 (s, 0.26H, CONHOCH${ }_{2} \mathrm{Ph}$ ), 8.87 (s, 0.23H, $\left.\mathrm{CONHOCH}_{2} \mathrm{Ph}\right) ;{ }^{13} \mathrm{C} \mathrm{NMR}$ $\left(50 \mathrm{MHz}, \mathrm{CDCl}_{3}\right) \delta 13.9\left(\mathrm{CH}_{3}\left(\mathrm{CH}_{2}\right)_{3}\right), 22.9\left(\mathrm{CH}_{3} \mathrm{CH}_{2}\left(\mathrm{CH}_{2}\right)_{2}\right), 25.9\left(\mathrm{CH}_{3} \mathrm{CH}_{2} \mathrm{CH}_{2} \mathrm{CH}_{2}\right), 39.5\left(\mathrm{CH}_{2} \mathrm{CONHOCH}_{2} \mathrm{Ph}\right), 41.6$ $\left(\mathrm{CH}_{3}\left(\mathrm{CH}_{2}\right)_{2} \mathrm{CH}_{2}\right), 46.0$ (5-C), 65.7 (3-C), 78.4, 79.6 ( $\left.\mathrm{CONHOCH}_{2} \mathrm{Ph}\right), 126.3,128.2,128.8,128.9,129.3,129.4,135.1$, 137.9 (aromatic C), $160.0\left(\mathrm{CONHOCH}_{2} \mathrm{Ph}\right), 171.7,173.4(2,6-\mathrm{C})$; EI MS: m/z $410.1\left([\mathrm{M}+\mathrm{H}]^{+}, 17\right), 409.1\left([\mathrm{M}]^{+}, 55\right)$, $352.1\left(\left[\mathrm{M}-\left(\mathrm{CH}_{2}\right)_{3} \mathrm{CH}_{3}\right]^{+}, 59\right), 318.1\left(\left[\mathrm{M}-\mathrm{CH}_{2} \mathrm{Ph}\right]^{+}, 100\right), 302.1\left(\left[\mathrm{M}-\mathrm{OCH}_{2} \mathrm{Ph}\right]^{+}, 47\right)$.

Compound 39 (900 mg, $2.2 \mathrm{mmol}$ ) was subjected to catalytic hydrogenation $\left(\mathrm{H}_{2} / 10 \% \mathrm{Pd}-\mathrm{C}, 108 \mathrm{mg}\right)$ in abs EtOH $(98 \mathrm{~mL})$ as described for the preparation of compound 6 from 37 . The crude hydrogenation product (glass oil) was chromatographed on silica gel column with AcOEt, as eluent, to afford the title compound 8 as a white crystalline solid (530 mg, 75\%): $\mathrm{mp} 158-160{ }^{\circ} \mathrm{C}$ (dec) ( $\left.\mathrm{MeOH}-\mathrm{Et}_{2} \mathrm{O}\right) ;{ }^{1} \mathrm{H} \mathrm{NMR}\left(600 \mathrm{MHz}, \mathrm{DMSO}-d_{6}\right) \delta 0.80(\mathrm{t}, 3 \mathrm{H}$, $\left.J=7.0 \mathrm{~Hz}, \mathrm{CH}_{3}\left(\mathrm{CH}_{2}\right)_{3}\right), 1.12-1.29$ (complex m, 4H, $\mathrm{CH}_{3} \mathrm{CH}_{2} \mathrm{CH}_{2} \mathrm{CH}_{2}$ ), 1.75-1.95(dm, 2H, $\left.\mathrm{CH}_{3}\left(\mathrm{CH}_{2}\right)_{2} \mathrm{CH}_{2}\right), 3.18-3.26(\mathrm{q}$, $1 \mathrm{H}, J=10.0 \mathrm{~Hz}, 4-\mathrm{H}), 3.58-3.64$ (dd, $1 \mathrm{H}, J=4.1,18.0 \mathrm{~Hz}, 5-\mathrm{H}), 3.69-3.75$ (dd, $1 \mathrm{H}, J=4.1,10.1 \mathrm{~Hz}, 5-\mathrm{H}), 4.17-4.28$ (q, $\mathrm{AB}, 1.6 \mathrm{H}, J_{A B}=15.5 \mathrm{~Hz}, \mathrm{CH}_{2} \mathrm{CONHOH}, E$-isomer), 4.47-4.57 (q, $\mathrm{AB}, 0.4 \mathrm{H}, J_{A B}=16.7 \mathrm{~Hz}, \mathrm{CH}_{2} \mathrm{CONHOH}, \mathrm{Z}$-isomer), 7.28$7.48(\mathrm{~m}, 5 \mathrm{H}$, aromatic $\mathrm{H}), 8.89(\mathrm{~s}, 0.7 \mathrm{H}, \mathrm{CONHOH}, E$-isomer), 9.30 (s, 0.2H, CONHOH, Z-isomer), $10.17(\mathrm{~s}, 0.2 \mathrm{H}$, $\mathrm{CONHOH}, \mathrm{Z}$-isomer), $10.60\left(\mathrm{~s}, 0.7 \mathrm{H}, \mathrm{CONHOH}, \mathrm{E}\right.$-isomer); ${ }^{13} \mathrm{C} \mathrm{NMR}\left(50 \mathrm{MHz}, \mathrm{DMSO}-d_{6}\right) \delta 13.9\left(\mathrm{CH}_{3}\left(\mathrm{CH}_{2}\right)_{3}\right), 22.4$ $\left(\mathrm{CH}_{3} \mathrm{CH}_{2}\left(\mathrm{CH}_{2}\right)_{2}\right), 25.5\left(\mathrm{CH}_{3} \mathrm{CH}_{2} \mathrm{CH}_{2} \mathrm{CH}_{2}\right), 38.9\left(\mathrm{CH}_{2} \mathrm{CONHOCH}_{2} \mathrm{Ph}\right), 41.0\left(\mathrm{CH}_{3}\left(\mathrm{CH}_{2}\right)_{2} \mathrm{CH}_{2}\right), 45.7$ (5-C), 65.0 (3-C), 126.2 , 127.6, 128.5 (2,3,4,5,6-aromatic C), 138.8 (1-aromatic C), 163.8 (CONHOH, E-isomer), 169.2 (CONHOH, Zisomer), 171.3, 173.1 (2,6-C); $\mathrm{ESI}^{+} \mathrm{MS}: \mathrm{m} / \mathrm{z} 342.3$ [M+Na] ${ }^{+}$. Anal. Calcd for $\mathrm{C}_{16} \mathrm{H}_{21} \mathrm{~N}_{3} \mathrm{O}_{4}$ : C, 60.17; $\mathrm{H}, 6.63 ; \mathrm{N}, 13.16$; Found: C, 60.25; $\mathrm{H}, 6.70 ; \mathrm{N}, 12.98$. The hydrochloride salt $(\mathbf{8} \cdot \mathbf{H C l})$ was prepared as described $\mathrm{for} 7 \cdot \mathbf{H C l}$ (decomposed gradually above $100^{\circ} \mathrm{C}$ ). Anal. Calcd for $\mathrm{C}_{16} \mathrm{H}_{22} \mathrm{ClN}_{3} \mathrm{O}_{4}$ : $\mathrm{C}, 54.01 ; \mathrm{H}, 6.23 ; \mathrm{N}, 11.81 ;$ Found: $\mathrm{C}, 53.63$; H, 5.92; N, 11.54 .

4.1.46. 3-(4-Fluorophenyl)-N-hydroxy-3-methyl-2,6-dioxo-1-piperazineacetamide 9

A stirred solution of carboxylic acid $36(750 \mathrm{mg}, 2.68 \mathrm{mmol}$ ) and 1,1'-carbonyldiimidazol (522 $\mathrm{mg}, 3.22 \mathrm{mmol})$ in dry THF $(53 \mathrm{~mL})$ was heated at $55^{\circ} \mathrm{C}$ for $1 \mathrm{~h}$ under argon. Then, $O$-benzylhydroxylamine hydrochloride $(514 \mathrm{mg}$, $3.22 \mathrm{mmol}$ ) was added followed by triethylamine (652 mg, $6.44 \mathrm{mmol}$ ), and the mixture was stirred at $55^{\circ} \mathrm{C}$ for $25 \mathrm{~h}$ under argon. The reaction was then worked up in the same way described in 37 . The resulting oily residue was purified by column chromatography eluting first with AcOEt- $n$-hexane $1: 2$ and then 1:1 to afford the 
corresponding $\mathrm{N}$-benzyloxy precursor $\mathbf{4 0}$ as a white foamy solid, which strongly binds the elution solvents. Removal of the entrapped solvents as in $\mathbf{3 7}$ gave $\mathbf{4 0}$ as a glass solid (632 mg, 61\%): This compound appears in the ${ }^{1} \mathrm{H}$ and ${ }^{13} \mathrm{C}$ NMR spectra as a mixture of $E / Z$ conformers (not assigned). ${ }^{1} \mathrm{H}$ NMR (400 MHz, $\mathrm{CDCl}_{3}$ ) $\delta 1.57$ (s, $\left.3 \mathrm{H}, \mathrm{CH}_{3}\right), 2.36-2.62$ (br s, $\left.1 \mathrm{H}, 4-\mathrm{H}\right), 3.38(\mathrm{t}, 1 \mathrm{H}, \mathrm{J} \simeq 18.4 \mathrm{~Hz}, 5-\mathrm{H}$ ), 3.64 (t like, $1 \mathrm{H}, \mathrm{J}=16.4,17.8 \mathrm{~Hz}, 5-\mathrm{H}$ ), 4.23-4.44 (q, $\mathrm{AB}, 1 \mathrm{H}, \mathrm{J}_{A B} \simeq 14.8 \mathrm{~Hz}, \mathrm{CH}_{2} \mathrm{CONHOCH}_{2} \mathrm{Ph}$ ), $4.68\left(\mathrm{~s}, 0.94 \mathrm{H}, \mathrm{CH}_{2} \mathrm{CONHOCH}_{2} \mathrm{Ph}\right.$ ), 4.86, 4.90 (s $+\mathrm{s}, 2 \mathrm{H}$, $\mathrm{CONHOCH}_{2} \mathrm{Ph}$ ), $7.04\left(\mathrm{t}, 2 \mathrm{H}, \mathrm{J}=8.4 \mathrm{~Hz}, 3,5-\mathrm{H}\right.$ for 4- $\mathrm{FC}_{6} \mathrm{H}_{4}$ ), 7.15-7.55 (complex m, 7H, $\mathrm{C}_{6} \mathrm{H}_{5}, 2,6-\mathrm{H}$ for 4- $\mathrm{FC}_{6} \mathrm{H}_{4}$ ), 8.60 (br s, 0.4H, CONHOCH${ }_{2} \mathrm{Ph}$ ), 9.30 (br s, 0.5H, $\left.\mathrm{CONHOCH}_{2} \mathrm{Ph}\right) ;{ }^{13} \mathrm{C} \mathrm{NMR}\left(50 \mathrm{MHz}, \mathrm{CDCl}_{3}\right) \delta 29.0\left(\mathrm{CH}_{3}\right), 39.6$ $\left(\mathrm{CH}_{2} \mathrm{CONHOCH}_{2} \mathrm{Ph}\right.$ ), 46.0 (5-C), 62.3 (3-C), 78.5, 79.7 ( $\left.\mathrm{CONHOCH}_{2} \mathrm{Ph}\right), 115.7,116.1$ (d, $J_{c-F}=21.3 \mathrm{~Hz}, 3,5-\mathrm{C}$ for 4$\left.\mathrm{FC}_{6} \mathrm{H}_{4}\right), 127.7,127.9\left(\mathrm{~d}, \mathrm{~J}_{\mathrm{C}-\mathrm{F}}=8.1 \mathrm{~Hz}, 2,6-\mathrm{C}\right.$ for $\left.4-\mathrm{FC}_{6} \mathrm{H}_{4}\right), 128.8,129.4\left(2,3,4,5,6-\mathrm{C}\right.$ for $\left.\mathrm{C}_{6} \mathrm{H}_{5}\right), 134.7,134.8$ (1-C for 4- $\mathrm{FC}_{6} \mathrm{H}_{4}$ and $\left.\mathrm{C}_{6} \mathrm{H}_{5}\right), 160.1,165.0$ (d, $\mathrm{J}_{C-\mathrm{F}}=246 \mathrm{~Hz}, 4-\mathrm{C}$ for $\left.4-\mathrm{FC}_{6} \mathrm{H}_{4}\right), 165.4,170.5\left(\mathrm{CONHOCH}_{2} \mathrm{Ph}\right.$, weak signal intensities), 171.7, 173.8 (2,6-C); ESI ${ }^{+} \mathrm{MS}: \mathrm{m} / \mathrm{z} 386.3[\mathrm{M}+\mathrm{H}]^{+}$.

Compound 40 (1 g, $2.59 \mathrm{mmol})$ was subjected to catalytic hydrogenation $\left(\mathrm{H}_{2} / 10 \% \mathrm{Pd}-\mathrm{C}, 120 \mathrm{mg}\right)$, in abs EtOH $(117 \mathrm{~mL}$ ) as described for the preparation of compound $\mathbf{6}$ from 37. The crude hydrogenation product (off-white foamy solid) was chromatographed on silica gel column with AcOEt, as eluent, to afford the title compound 9 as a white foamy solid, which gave white crystals upon trituration with $\mathrm{Et}_{2} \mathrm{O}(720 \mathrm{mg}, 94 \%): \mathrm{mp} 154-158^{\circ} \mathrm{C}$ (dec) (AcOEt-Et ${ }_{2} \mathrm{O}$ ); ${ }^{1} \mathrm{H}$ NMR (400 MHz, DMSO-d $\left.d_{6}\right) \delta 1.49\left(\mathrm{~s}, 3 \mathrm{H}, \mathrm{CH}_{3}\right.$ ), 3.02-3.19 (q, $1 \mathrm{H}, \mathrm{J}=10.7 \mathrm{~Hz}, 5-\mathrm{H}$ ), 3.52-3.65 (dd, $1 \mathrm{H}, \mathrm{J}=3.9,18.3 \mathrm{~Hz}, 5-\mathrm{H}$ ), 3.80-3.95 (dd, $1 \mathrm{H}, \mathrm{J} \simeq 3.8,10.5 \mathrm{~Hz}, 4-\mathrm{H}), 4.24$ (s, 1.5H, $\mathrm{CH}_{2} \mathrm{CONHOH}, E$-isomer), 4.54 (s, $0.5 \mathrm{H}, \mathrm{CH}_{2} \mathrm{CONHOH}, \mathrm{Z}$-isomer), 7.19 (t, $2 \mathrm{H}, J=8.7 \mathrm{~Hz}, 3,5$-aromatic $\mathrm{H}$ ), 7.51 (t like, $2 \mathrm{H}, J=5.6,8.1 \mathrm{~Hz}, 2$,6-aromatic $\mathrm{H}), 8.96(\mathrm{~s}, 0.8 \mathrm{H}, \mathrm{CONHOH}, \mathrm{E}$-isomer), 9.37 (s, 0.2H, CONHOH, Z-isomer), 10.25 (s, 0.2H, CONHOH, Z-isomer), $10.67\left(\mathrm{~s}, 0.8 \mathrm{H}, \mathrm{CONHOH}, E\right.$-isomer); ${ }^{13} \mathrm{C} \mathrm{NMR}\left(50 \mathrm{MHz}, \mathrm{DMSO}-d_{6}\right) \delta 28.3\left(\mathrm{CH}_{3}\right), 39.0\left(\mathrm{CH}_{2} \mathrm{CONHOH}, E\right.$-isomer), 39.5 ( $\mathrm{CH}_{2} \mathrm{CONHOH}, \mathrm{Z}$-isomer), 45.6 (5-C), 61.7 (3-C), 115.2, 115.6 (d, $J_{C-F}=21.1 \mathrm{~Hz}, 3,5$-aromatic C), 128.2, 128.3 (d, $J_{C-F}=7.5 \mathrm{~Hz}, 2,6$-aromatic C), 136.4 (1-aromatic C), 159.2, 164.0 (d, $J_{C-F}=242 \mathrm{~Hz}$, 4-aromatic C), 163.9 (CONHOH, E-isomer), 169.2 (CONHOH, Z-isomer), 171.4, 173.6 (2,6-C); Anal. Calcd for $\mathrm{C}_{13} \mathrm{H}_{14} \mathrm{FN}_{3} \mathrm{O}_{4}$ : C, 52.88; $\mathrm{H}, 4.78 ; \mathrm{N}$, 14.23; Found: $\mathrm{C}, 52.67 ; \mathrm{H}, 4.88 ; \mathrm{N}, 14.32$. The hydrochloride salt was prepared by treating an ethyl acetate solution of 9 with saturated solution of $\mathrm{HCl}$ in $\mathrm{Et}_{2} \mathrm{O}$ under ice cooling. The white precipitate was collected by vacuum filtration, triturated with $\mathrm{Et}_{2} \mathrm{O}$ and dried (decomposed gradually above $120{ }^{\circ} \mathrm{C}$ ). Anal. Calcd for $\mathrm{C}_{13} \mathrm{H}_{15} \mathrm{ClFN}_{3} \mathrm{O}_{4}$ : C, 47.07; $\mathrm{H}, 4.56 ; \mathrm{N}, 12.67$; Found: $\mathrm{C}, 46.70 ; \mathrm{H}, 4.48 ; \mathrm{N}, 12.35$.

4.1.47. $\mathrm{N}$-Hydroxy-3-methyl-3-(4-nitrophenyl)-2,6-dioxo-1-piperazineacetamide Hydrochloride 10

4-Methoxybenzyl ester $32(1 \mathrm{~g}, 2.34 \mathrm{mmol})$ was taken in $\mathrm{CH}_{2} \mathrm{Cl}_{2}(26 \mathrm{~mL})$ and treated with trifluoroacetic acid (4 $\mathrm{mL}$ ). The mixture was left stirring at room temperature for $90 \mathrm{~min}$, evaporated to dryness in vacuo, and dissolved in dry THF (46 mL). Triethylamine (474 mg, $4.68 \mathrm{mmol}$ ) and 1,1'-carbonyldiimidazol (456 mg, $2.81 \mathrm{mmol}$ ) were then added, and the mixture was stirred at $28^{\circ} \mathrm{C}$ for $1 \mathrm{~h}$ under argon. After this time, 0 -(4-methoxybenzyl) hydroxylamine ( $430 \mathrm{mg}, 2.81 \mathrm{mmol}$ ) was added, and the resulting mixture was stirred for $18 \mathrm{~h}$ at $28^{\circ} \mathrm{C}$ and $7 \mathrm{~h}$ at $55{ }^{\circ} \mathrm{C}$ under argon. The solvent was removed under reduced pressure, and the residue was partitioned between ethyl acetate $(100 \mathrm{~mL}$ ) and brine $(50 \mathrm{~mL})$. The aqueous layer was extracted once more with ethyl acetate $(50 \mathrm{~mL})$, and the combined organic extracts were washed once with brine $(50 \mathrm{~mL})$, dried $\left(\mathrm{Na}_{2} \mathrm{SO}_{4}\right)$ and evaporated in vacuo. The resulting yellow thick oil was purified by column chromatography eluting with AcOEt$n$-hexane 1:1 to 4:1 to afford the $N$-(4-methoxybenzyloxy) precursor 41 as a yellowish foamy solid, which strongly binds the elution solvents. Removal of the entrapped solvents as in $\mathbf{3 7}$ gave $\mathbf{4 1}$ as a glass solid (500 mg, $48 \%$ yield over two steps). This compound appears in the ${ }^{1} \mathrm{H}$ and ${ }^{13} \mathrm{C} N \mathrm{NR}$ spectra as a mixture of $E / Z$ conformers (not assigned). ${ }^{1} \mathrm{H}$ NMR $\left(400 \mathrm{MHz}, \mathrm{CDCl}_{3}\right) \delta 1.64\left(\mathrm{~s}, 3 \mathrm{H}, \mathrm{CH}_{3}\right), 2.25-2.75$ (br s, $\left.1 \mathrm{H}, 4-\mathrm{H}\right), 3.28-3.47(\mathrm{~m}, 1 \mathrm{H}, 5-\mathrm{H})$, $\left.3.72(\mathrm{~d}, 1 \mathrm{H}, \mathrm{J}=18.8 \mathrm{~Hz}, 5-\mathrm{H}), 3.80(\mathrm{~s}, 3 \mathrm{H}, \mathrm{OCH})_{3}\right), 4.27-4.47\left(\mathrm{q}, \mathrm{AB}, 0.9 \mathrm{H}, \mathrm{J}_{\mathrm{AB}} \simeq 15.0 \mathrm{~Hz}, \mathrm{CH}_{2} \mathrm{CONHOCH}_{2} \mathrm{C}_{6} \mathrm{H}_{4} \mathrm{OCH}_{3}-4\right)$, $4.66\left(\mathrm{~s}, 1.1 \mathrm{H}, \mathrm{CH}_{2} \mathrm{CONHOCH}_{2} \mathrm{C}_{6} \mathrm{H}_{4} \mathrm{OCH}_{3}-4\right), 4.82,4.84$ (s + s, 2H, $\left.\mathrm{CONHOCH}_{2} \mathrm{C}_{6} \mathrm{H}_{4} \mathrm{OCH}_{3}-4\right), 6.89$ (s, 2H, 3,5-H for 4$\left.\mathrm{CH}_{3} \mathrm{OC}_{6} \mathrm{H}_{4}\right), 7.32\left(\mathrm{~d}, 2 \mathrm{H}, \mathrm{J}=8.5 \mathrm{~Hz}, 2,6-\mathrm{H}\right.$ for $\left.4-\mathrm{CH}_{3} \mathrm{OC}_{6} \mathrm{H}_{4}\right), 7.72\left(\mathrm{~d}, 2 \mathrm{H}, \mathrm{J}=7.4 \mathrm{~Hz}, 2,6-\mathrm{H}\right.$ for 4- $\left.\mathrm{NO}_{2} \mathrm{C}_{6} \mathrm{H}_{4}\right), 8.21$ (d, $2 \mathrm{H}$, $J=8.8 \mathrm{~Hz}, 3,5-\mathrm{H}$ for $\left.4-\mathrm{NO}_{2} \mathrm{C}_{6} \mathrm{H}_{4}\right), 8.44\left(\mathrm{~s}, 0.43 \mathrm{H}, \mathrm{CONHOCH}_{2} \mathrm{C}_{6} \mathrm{H}_{4} \mathrm{OCH}_{3}-4\right), 9.15\left(\mathrm{~s}, 0.4 \mathrm{H}, \mathrm{CONHOCH}_{2} \mathrm{C}_{6} \mathrm{H}_{4} \mathrm{OCH}_{3}-4\right)$; ${ }^{13} \mathrm{C} \mathrm{NMR}\left(50 \mathrm{MHz}, \mathrm{CDCl}_{3}\right) \delta 29.0\left(3-\mathrm{CH}_{3}\right), 39.7\left(\mathrm{CH}_{2} \mathrm{CONHOCH}_{2} \mathrm{C}_{6} \mathrm{H}_{4} \mathrm{OCH}_{3}-4\right), 46.0(5-\mathrm{C}), 55.5\left(\mathrm{OCH}_{3}\right), 62.7(3-\mathrm{C})$, 
78.2, 79.4 ( $\left.\mathrm{CONHOCH}_{2} \mathrm{C}_{6} \mathrm{H}_{4} \mathrm{OCH}_{3}-4\right), 114.3,124.3,127.3,131.3,146.5,147.9,160.4$ (aromatic C), $164.9,170.1$ $\left(\mathrm{CONHOCH}_{2} \mathrm{C}_{6} \mathrm{H}_{4} \mathrm{OCH}_{3}-4\right), 171.0,173.0(2,6-\mathrm{C}) ; \mathrm{ESI}^{+} \mathrm{MS}: \mathrm{m} / \mathrm{z} 443.6[\mathrm{M}+\mathrm{H}]^{+}$.

A solution of compound 41 (500 mg, $1.13 \mathrm{mmol}$ ) and trifluoroacetic acid $(8.3 \mathrm{~mL})$ in $\mathrm{CH}_{2} \mathrm{Cl}_{2}$ (32 $\left.\mathrm{mL}\right)$ was stirred for $10 \mathrm{~min}$, and subsequently treated dropwise with $\mathrm{Et}_{3} \mathrm{SiH}(830 \mu \mathrm{L})$ via syringe. After $45 \mathrm{~min}$ of stirring at room temperature, the color changed from ruby to pale yellow, and the mixture was evaporated to dryness under reduced pressure. A slurry of sodium chloride in water $(3 \mathrm{~mL})$ was added to the residue, and the mixture was treated slowly with solid $\mathrm{Na}_{2} \mathrm{CO}_{3}$ to $\mathrm{pH}$ 8-9. This thick mixture was then washed five times with ethyl acetate (15 $\mathrm{mL}$ ) under vigorous stirring, and the combined washings were dried $\left(\mathrm{Na}_{2} \mathrm{SO}_{4}\right)$ and concentrated to dryness in vacuo. The resulting oily residue was chromatographed on silica gel column with AcOEt, as eluent, to afford the free base 10 as a yellowish foamy solid (HRMS (ESI): $[\mathrm{M}+\mathrm{H}]^{+}$calcd for $\mathrm{C}_{13} \mathrm{H}_{14} \mathrm{~N}_{4} \mathrm{O}_{6}, 323.0992$, found 323.0994). This free base material was dissolved in $\mathrm{AcOEt}_{-} \mathrm{Et}_{2} \mathrm{O} 1: 1$ and treated with saturated solution of $\mathrm{HCl}$ in $\mathrm{Et}_{2} \mathrm{O}$ under ice cooling. The resulting precipitate was collected by vacuum filtration, triturated with $\mathrm{Et}_{2} \mathrm{O}$, and dried in vacuo to give $256 \mathrm{mg}$ (63\%) of the title compound $\mathbf{1 0}$ as an off-white hygroscopic solid (decomposed gradually above $\left.160{ }^{\circ} \mathrm{C}\right) ;{ }^{1} \mathrm{H}$ NMR (400 MHz, DMSO- $\left.d_{6}\right) \delta 1.62\left(\mathrm{~s}, 3 \mathrm{H}, \mathrm{CH}_{3}\right), 3.28(\mathrm{~d}, 1 \mathrm{H}, J=18.4 \mathrm{~Hz}, 5-\mathrm{H}), 3.76(\mathrm{~d}, 1 \mathrm{H}, J=18.4 \mathrm{~Hz}, 5-$ $\mathrm{H}), 4.27\left(\mathrm{~s}, 1.5 \mathrm{H}, \mathrm{CH}_{2} \mathrm{CONHOH}, \mathrm{E}\right.$-isomer), 4.55 (s, 0.5H, $\mathrm{CH}_{2} \mathrm{CONHOH}, Z$-isomer), 4.19-5.30 ( $\mathrm{v} \mathrm{br} \mathrm{s,} \mathrm{NH}_{2}^{+}$, CONHOH, under DMSO water peak), 7.82 (d, $2 \mathrm{H}, J=8.4 \mathrm{~Hz}, 2$,6-aromatic $\mathrm{H}$ ), 8.24 (d, $2 \mathrm{H}, J=8.8 \mathrm{~Hz}, 3,5$-aromatic $\mathrm{H}), 10.31$ (s, 0.2H, CONHOH, Z-isomer), $10.78\left(\mathrm{~s}, 0.6 \mathrm{H}, \mathrm{CONHOH}, E\right.$-isomer); ${ }^{13} \mathrm{C}$ NMR (50 MHz, DMSO- $\left.d_{6}\right) \delta 25.9$ $\left(\mathrm{CH}_{3}\right), 39.8\left(\mathrm{CH}_{2} \mathrm{CONHOH}\right), 44.1(5-\mathrm{C}), 62.6(3-\mathrm{C}), 123.9,128.4,144.6,147.6$ (aromatic C), $163.4(\mathrm{CONHOH}, E-$ isomer), 168.7 (CONHOH, Z-isomer), 168.0, 170.6 (2,6-C). Anal. Calcd for $\mathrm{C}_{13} \mathrm{H}_{15} \mathrm{ClN}_{4} \mathrm{O}_{6}: \mathrm{C}, 43.52 ; \mathrm{H}, 4.21 ; \mathrm{N}$, 15.62; Found: C, 43.08; H, 4.46; N, 15.91.

4.1.48. $\mathrm{N}$-Hydroxy-3,4-dimethyl-2,6-dioxo-3-phenyl-1-piperazineacetamide 11

The $N$-benzyloxy precursor 61 was prepared from carboxylic acid 57 (680 mg, 2.46 mmol) following the procedure described for the preparation of compound 37 (precursor for $\mathbf{6}$ ). The crude oil was purified by flash column chromatography eluting with AcOEt- $n$-hexane $1: 1$ to afford 61 as a clear glass oil (700 mg, $75 \%$ ). This compound appears in the ${ }^{1} \mathrm{H}$ and ${ }^{13} \mathrm{C}$ NMR spectra as a mixture of $E / Z$ conformers (not assigned). ${ }^{1} \mathrm{H} N M R(600$ $\mathrm{MHz}, \mathrm{CDCl}_{3}, 283 \mathrm{~K}$ ) $\delta 1.65$ (s , 3H, 3- $\left.\mathrm{CH}_{3}\right), 2.57,2.58\left(\mathrm{~s}+\mathrm{s}, 3 \mathrm{H}, 4-\mathrm{CH}_{3}\right), 3.36-3.44(\mathrm{dd}, 1 \mathrm{H}, \mathrm{J}=8.4,20.4 \mathrm{~Hz}, 5-\mathrm{H}), 3.65$ (t, $1 \mathrm{H}, J=15.6,16.8 \mathrm{~Hz}, 5-\mathrm{H}), 4.30-4.43\left(\mathrm{q}, \mathrm{AB}, 0.96 \mathrm{H}, J_{A B}=15.0 \mathrm{~Hz}, \mathrm{CH}_{2} \mathrm{CONHOCH}_{2} \mathrm{Ph}\right), 4.75(\mathrm{~d}, 1.1 \mathrm{H}, J=4.2 \mathrm{~Hz}$, $\mathrm{CH}_{2} \mathrm{CONHOCH}_{2} \mathrm{Ph}$ ), 4.89, $4.92\left(\mathrm{~s}+\mathrm{s}, 2 \mathrm{H}, \mathrm{CONHOCH}_{2} \mathrm{Ph}\right.$ ), 7.27-7.50 (complex m, 10H, aromatic $\mathrm{H}$ ), $8.52(\mathrm{~s}, 0.45 \mathrm{H}$, $\mathrm{CONHOCH}_{2} \mathrm{Ph}$ ), 9.0 (s, 0.44H, $\left.\mathrm{CONHOCH}_{2} \mathrm{Ph}\right) ;{ }^{13} \mathrm{C} \mathrm{NMR}\left(150 \mathrm{MHz}, \mathrm{CDCl}_{3}, 283 \mathrm{~K}\right) \delta 24.2\left(3-\mathrm{CH}_{3}\right), 38.1\left(4-\mathrm{CH}_{3}\right), 39.1$, $39.5\left(\mathrm{CH}_{2} \mathrm{CONHOCH}_{2} \mathrm{Ph}\right), 54.9$ (5-C), 67.8 (3-C), 78.1, $79.5\left(\mathrm{CONHOCH}_{2} \mathrm{Ph}\right), 125.9,126.0,128.0,128.1,128.4$, 128.5, 128.6, 128.8, 129.1, 129.3, 134.0, 135.1, 140.5 (aromatic C), 165.0, 170.2 (CONHOCH $\left.{ }_{2} \mathrm{Ph}\right), 170.5,173.7$ (2,6-C); HRMS (ESI): $[\mathrm{M}+\mathrm{H}]^{+}$calcd for $\mathrm{C}_{21} \mathrm{H}_{23} \mathrm{~N}_{3} \mathrm{O}_{4}, 382.1767$, found 382.1753.

Compound 61 (720 mg, $1.89 \mathrm{mmol}$ ) was subjected to catalytic hydrogenation $\left(\mathrm{H}_{2} / 10 \% \mathrm{Pd}-\mathrm{C}, 86 \mathrm{mg}\right)$ in abs EtOH $(85 \mathrm{~mL}$ ) as described for the preparation of compound 6 from 37. The crude hydrogenation product (off-white foamy solid) was chromatographed over flash silica eluting with AcOEt to afford the title compound $\mathbf{1 1}$ as a white foamy solid, which strongly binds the aforementioned solvent. Removal of the entrapped solvent as in 6 gave 11 as a slightly off-yellow solid (495 mg, 90\%): $\mathrm{mp} 158-160{ }^{\circ} \mathrm{C}$ (dec); ${ }^{1} \mathrm{H}$ NMR (400 MHz, DMSO- $\left.d_{6}\right) \delta 1.56$ $\left(\mathrm{s}, 3 \mathrm{H}, 3-\mathrm{CH}_{3}\right), 2.39,2.41$ ( $\left.\mathrm{s}+\mathrm{s}, 3 \mathrm{H}, 4-\mathrm{CH}_{3}\right), 3.31-3.59$ (q, $\left.\mathrm{AB}, 2 \mathrm{H}, J_{A B}=17.9 \mathrm{~Hz}, 5-\mathrm{H}\right), 4.29$ (t like, $1.47 \mathrm{H}, \mathrm{J}=16.0 \mathrm{~Hz}$, $\mathrm{CH}_{2} \mathrm{CONHOH}, \mathrm{E}$-isomer), 4.59 (s, 0.46H, $\mathrm{CH}_{2} \mathrm{CONHOH}, \mathrm{Z}$-isomer), 7.28-7.50 (complex m, 5H, aromatic $\mathrm{H}$ ), 8.95 (s, 0.7H, CONHOH, E-isomer), 9.38 (s, 0.2H, CONHOH, Z-isomer), 10.27 (s, 0.2H, CONHOH, Z-isomer), 10.73 (s, $0.7 \mathrm{H}$, $\mathrm{CONHOH}, E$-isomer); ${ }^{13} \mathrm{C} \mathrm{NMR}\left(100 \mathrm{MHz}, \mathrm{DMSO}-d_{6}\right) \delta 22.4,22.5\left(3-\mathrm{CH}_{3}\right), 37.3\left(4-\mathrm{CH}_{3}\right), 38.8\left(\mathrm{CH}_{2} \mathrm{CONHOH}, E-\right.$ isomer), 39.7 ( $\mathrm{CH}_{2} \mathrm{CONHOH}, Z$-isomer), 54.0 (5-C), 67.3 (3-C), 126.5, 127.9, 128.5 (2,3,4,5,6-aromatic C), 140.7 (1-aromatic C), 163.8 (CONHOH, E-isomer), 169.2 (CONHOH, Z-isomer), 169.8, 169.9, 173.5 (2,6-C); HRMS (ESI): $[\mathrm{M}+\mathrm{H}]^{+}$calcd for $\mathrm{C}_{14} \mathrm{H}_{17} \mathrm{~N}_{3} \mathrm{O}_{4}, 292.1297$, found 292.1295. The hydrochloride salt $(\mathbf{1 1} \cdot \mathrm{HCl})$ was prepared as described for $\mathbf{6} \cdot \mathbf{H C l}$. Mp $153-156{ }^{\circ} \mathrm{C}$ (dec) (slightly hygroscopic). Anal. Calcd for $\mathrm{C}_{14} \mathrm{H}_{18} \mathrm{ClN}_{3} \mathrm{O}_{4}: \mathrm{C}, 51.30 ; \mathrm{H}, 5.54$; N, 12.82; Found: C, 50.94; H, 5.92; N, 12.51. 


\subsubsection{9. $\mathrm{N}$-Hydroxy-4-methyl-2,6-dioxo-3-phenyl-3-propyl-1-piperazineacetamide 12}

The $\mathrm{N}$-benzyloxy precursor 62 was prepared from carboxylic acid $58(840 \mathrm{mg}, 2.76 \mathrm{mmol})$ following the procedure described for the preparation of compound $\mathbf{3 7}$ (precursor for $\mathbf{6}$ ). The crude viscous oil was purified by column chromatography on silica gel with AcOEt- $n$-hexane 2:3, as eluent, to afford 62 as a glass solid (790 $\mathrm{mg}, 69 \%)$. This compound appears in the ${ }^{1} \mathrm{H}$ and ${ }^{13} \mathrm{C} \mathrm{NMR} \mathrm{spectra} \mathrm{as} \mathrm{a} \mathrm{mixture} \mathrm{of} E / Z$ conformers (not assigned). ${ }^{1} \mathrm{H} \mathrm{NMR}\left(400 \mathrm{MHz}, \mathrm{CDCl}_{3}\right) \delta 0.73\left(\mathrm{t}, 3 \mathrm{H}, \mathrm{J}=7.1 \mathrm{~Hz}, \mathrm{CH}_{3}\left(\mathrm{CH}_{2}\right)_{2}\right), 1.02-1.20\left(\mathrm{~m}, 2 \mathrm{H}, \mathrm{CH}_{3} \mathrm{CH}_{2} \mathrm{CH}_{2}\right), 1.76-2.10(\mathrm{dm}, 2 \mathrm{H}$, $\mathrm{CH}_{3} \mathrm{CH}_{2} \mathrm{CH}_{2}$ ), $2.44\left(\mathrm{~s}, 3 \mathrm{H}, 4-\mathrm{CH}_{3}\right), 3.23-3.70\left(\mathrm{q}, \mathrm{AB}, 2 \mathrm{H}, \mathrm{J}_{\mathrm{AB}}=18.0 \mathrm{~Hz}, 5-\mathrm{H}\right), 4.12-4.37$ ( br s, $1 \mathrm{H}, \mathrm{CH}_{2} \mathrm{CONHOCH}_{2} \mathrm{Ph}$ ), 4.55, 4.61 (s s s, 0.9H, $\mathrm{CH}_{2} \mathrm{CONHOCH}_{2} \mathrm{Ph}$ ), $4.78\left(\mathrm{~s}, 2 \mathrm{H}, \mathrm{CONHOCH}_{2} \mathrm{Ph}\right.$ ), 7.08-7.40 (complex m, 10H, aromatic H), 8.67-8.86 (br s, $0.32 \mathrm{H}, \mathrm{CONHOCH}_{2} \mathrm{Ph}$ ), 9.24-9.45 (br s, 0.41H, $\left.\mathrm{CONHOCH}_{2} \mathrm{Ph}\right) ;{ }^{13} \mathrm{C} \mathrm{NMR}\left(50 \mathrm{MHz}, \mathrm{CDCl}_{3}\right) \delta 14.2$ $\left(\mathrm{CH}_{3}\left(\mathrm{CH}_{2}\right)_{2}\right), 16.2\left(\mathrm{CH}_{3} \mathrm{CH}_{2} \mathrm{CH}_{2}\right), 37.7\left(4-\mathrm{CH}_{3}\right), 38.8\left(\mathrm{CH}_{3} \mathrm{CH}_{2} \mathrm{CH}_{2}\right), 39.4\left(\mathrm{CH}_{2} \mathrm{CONHOCH}_{2} \mathrm{Ph}\right), 55.0(5-\mathrm{C}), 70.3$ (3-C), 78.2, $79.5\left(\mathrm{CONHOCH}_{2} \mathrm{Ph}\right), 127.0,128.0,128.5,129.2,134.4,135.3,136.9$ (aromatic C), 165.2, 170.5, 173.7 (CONHOCH$\left.{ }_{2} \mathrm{Ph}, 2,6-\mathrm{C}\right)$; El MS: m/z $410.3\left([\mathrm{M}+\mathrm{H}]^{+}, 2\right), 409.2\left([\mathrm{M}]^{+}, 9\right), 367.2\left(\left[\mathrm{M}+\mathrm{H}-\mathrm{CH}_{2} \mathrm{CH}_{2} \mathrm{CH}_{3}\right]^{+}, 21\right), 366.2$ ([M$\left.\left.\mathrm{CH}_{2} \mathrm{CH}_{2} \mathrm{CH}_{3}\right]^{+}, 100\right), 338.2$ (22), 231.2 (79), 174.1 (93), 91.0 (16).

Compound 62 ( $1.33 \mathrm{~g}, 3.25 \mathrm{mmol}$ ) was subjected to catalytic hydrogenation $\left(\mathrm{H}_{2} / 10 \% \mathrm{Pd}-\mathrm{C}, 160 \mathrm{mg}\right)$ in abs EtOH $(146 \mathrm{~mL}$ ) as described for the preparation of compound 6 from 37 . The crude hydrogenation product (off-white foamy solid) was chromatographed on silica gel column with AcOEt, as eluent, to afford the title compound 12 as a white foamy solid, which strongly binds the elution solvent. Removal of the entrapped solvent as in $\mathbf{6}$ gave 12 as a glass solid (903 mg, 87\%): ${ }^{1} \mathrm{H}$ NMR (400 MHz, DMSO- $\left.d_{6}\right) \delta 0.81\left(\mathrm{t}, 3 \mathrm{H}, J=7.2 \mathrm{~Hz}, \mathrm{CH}_{3}\left(\mathrm{CH}_{2}\right)_{2}\right), 1.0-1.32$ (dm, $2 \mathrm{H}, \mathrm{CH}_{3} \mathrm{CH}_{2} \mathrm{CH}_{2}$ ), 1.87 (t, $1 \mathrm{H}, J=10.8,11.8 \mathrm{~Hz}, \mathrm{CH}_{3} \mathrm{CH}_{2} \mathrm{CHH}$ ), 2.07-2.23 (td, $1 \mathrm{H}, J=2.0,4.2,12.6,13.6 \mathrm{~Hz}$, $\mathrm{CH}_{3} \mathrm{CH}_{2} \mathrm{CHH}$ ), 2.37, 2.38 (s + s, 3H, 4- $\left.\mathrm{CH}_{3}\right), 3.26-3.57$ (q, AB, $\left.2 \mathrm{H}, \mathrm{J}_{A B}=18.0 \mathrm{~Hz}, 5-\mathrm{H}\right), 4.29$ (s, 1.3H, $\mathrm{CH}_{2} \mathrm{CONHOH}, E-$ isomer), $4.58\left(\mathrm{~s}, 0.5 \mathrm{H}, \mathrm{CH}_{2} \mathrm{CONHOH}, \mathrm{Z}\right.$-isomer), 7.27-7.47 (m, $5 \mathrm{H}$, aromatic $\left.\mathrm{H}\right), 8.97(\mathrm{~s}, 0.6 \mathrm{H}, \mathrm{CONHOH}, \mathrm{E}$-isomer), 9.40 (s, 0.2H, CONHOH, Z-isomer), 10.27 (s, 0.2H, CONHOH, Z-isomer), 10.74 (s, 0.6H, CONHOH, E-isomer); ${ }^{13} \mathrm{C}$ NMR $(50 \mathrm{MHz} \text {, DMSO-d })_{6} \delta 14.1\left(\mathrm{CH}_{3}\left(\mathrm{CH}_{2}\right)_{2}\right), 16.4\left(\mathrm{CH}_{3} \mathrm{CH}_{2} \mathrm{CH}_{2}\right), 36.9\left(4-\mathrm{CH}_{3}\right), 37.7\left(\mathrm{CH}_{3} \mathrm{CH}_{2} \mathrm{CH}_{2}\right), 39.0$ ( $\mathrm{CH}_{2} \mathrm{CONHOH}, E$-isomer), $39.8\left(\mathrm{CH}_{2} \mathrm{CONHOH}, Z\right.$-isomer), 53.9 (5-C), 70.0 (3-C), 127.2, 127.9, 128.3 (2,3,4,5,6aromatic C), 136.2 (1-aromatic C), 163.8 (CONHOH, E-isomer), 169.2 (CONHOH, Z-isomer), 169.7, 173.3 (2,6-C); HRMS (ESI): $[\mathrm{M}+\mathrm{H}]^{+}$calcd for $\mathrm{C}_{16} \mathrm{H}_{21} \mathrm{~N}_{3} \mathrm{O}_{4}, 320.1610$, found 320.1617 . The hydrochloride salt $(\mathbf{1 2} \cdot \mathrm{HCl})$ was prepared by treating a diethyl ether solution of $\mathbf{1 2}$ with saturated solution of $\mathrm{HCl}$ in $\mathrm{Et}_{2} \mathrm{O}$ under ice cooling. The resulting white solid was collected by vacuum filtration, triturated with $\mathrm{Et}_{2} \mathrm{O}$, and dried in vacuo (decomposed gradually above $110{ }^{\circ} \mathrm{C}$ ). Anal. Calcd for $\mathrm{C}_{16} \mathrm{H}_{22} \mathrm{ClN}_{3} \mathrm{O}_{4}$ : $\mathrm{C}, 54.01 ; \mathrm{H}, 6.23 ; \mathrm{N}, 11.81$; Found: $\mathrm{C}, 53.65 ; \mathrm{H}, 5.96 ; \mathrm{N}$, 11.62.

\subsubsection{3-Butyl-N-hydroxy-4-methyl-2,6-dioxo-3-phenyl-1-piperazineacetamide 13}

The $N$-benzyloxy precursor $\mathbf{6 3}$ was prepared from carboxylic acid $\mathbf{5 9}(1.2 \mathrm{~g}, 3.77 \mathrm{mmol})$ following the procedure described for the preparation of compound $\mathbf{3 7}$ (precursor for $\mathbf{6}$ ). The crude yellowish oil was purified by flash column chromatography eluting with AcOEt- $n$-hexane 2:3 to afford 63 as a white foamy solid, which strongly binds the elution solvents. Removal of the entrapped solvents as in $\mathbf{3 7}$ gave $\mathbf{6 3}$ as a glass solid $(1 \mathrm{~g}, 63 \%)$. This compound appears in the ${ }^{1} \mathrm{H}$ and ${ }^{13} \mathrm{C} N M R$ spectra as a mixture of $E / Z$ conformers (not assigned). ${ }^{1} \mathrm{H}$ NMR (600 $\left.\mathrm{MHz}, \mathrm{CDCl}_{3}\right) \delta 0.74\left(\mathrm{t}, 3 \mathrm{H}, J=7.1 \mathrm{~Hz}, \mathrm{CH}_{3}\left(\mathrm{CH}_{2}\right)_{3}\right), 1.03-1.21\left(\mathrm{~m}, 4 \mathrm{H}, \mathrm{CH}_{3} \mathrm{CH}_{2} \mathrm{CH}_{2} \mathrm{CH}_{2}\right), 1.84-2.10(\mathrm{dm}, 2 \mathrm{H}$, $\mathrm{CH}_{3}\left(\mathrm{CH}_{2}\right)_{2} \mathrm{CH}_{2}$ ), 2.46 (s, 3H, 4- $\mathrm{CH}_{3}$ ), 3.26-3.68 (q, $\left.\mathrm{AB}, 2 \mathrm{H}, \mathrm{J}_{\mathrm{AB}}=18.0 \mathrm{~Hz}, 5-\mathrm{H}\right), 4.18-4.38$ (br d, $1 \mathrm{H}, \mathrm{J}=24.0 \mathrm{~Hz}$, $\mathrm{CH}_{2} \mathrm{CONHOCH}_{2} \mathrm{Ph}$ ), 4.53-4.69 (br s, $1 \mathrm{H}, \mathrm{CH}_{2} \mathrm{CONHOCH}_{2} \mathrm{Ph}$ ), 4.81 (s, $2 \mathrm{H}, \mathrm{CONHOCH}_{2} \mathrm{Ph}$ ), 7.14-7.38 (complex m, $10 \mathrm{H}$, aromatic $\mathrm{H}$ ), 8.40-8.65 (br s, 0.6H, $\mathrm{CONHOCH}_{2} \mathrm{Ph}$ ), 8.85-9.10 (br s, 0.6H, CONHOCH${ }_{2} \mathrm{Ph}$ ); ${ }^{13} \mathrm{C} \mathrm{NMR} \mathrm{(50} \mathrm{MHz,}$ $\left.\mathrm{CDCl}_{3}\right) \delta 14.0\left(\mathrm{CH}_{3}\left(\mathrm{CH}_{2}\right)_{3}\right), 22.9\left(\mathrm{CH}_{3} \mathrm{CH}_{2}\left(\mathrm{CH}_{2}\right)_{2}\right), 24.9\left(\mathrm{CH}_{3} \mathrm{CH}_{2} \mathrm{CH}_{2} \mathrm{CH}_{2}\right), 36.4\left(\mathrm{CH}_{3}\left(\mathrm{CH}_{2}\right)_{2} \mathrm{CH}_{2}\right), 37.8\left(4-\mathrm{CH}_{3}\right), \quad 39.5$ $\left(\mathrm{CH}_{2} \mathrm{CONHOCH}_{2} \mathrm{Ph}\right), 55.1$ (5-C), 70.3 (3-C), 78.3, 79.5 ( $\left.\mathrm{CONHOCH}_{2} \mathrm{Ph}\right), 127.1,128.1,128.6,129.3,134.4,135.3$, 136.8 (aromatic C), 165.2, 170.6, $173.8\left(\mathrm{CONHOCH}_{2} \mathrm{Ph}, 2,6-\mathrm{C}\right) ; \mathrm{ESI}^{+} \mathrm{MS}: \mathrm{m} / \mathrm{z} 424.4[\mathrm{M}+\mathrm{H}]^{+}$.

Compound 63 (900 mg, $2.13 \mathrm{mmol}$ ) was subjected to catalytic hydrogenation $\left(\mathrm{H}_{2} / 10 \% \mathrm{Pd}-\mathrm{C}, 108 \mathrm{mg}\right)$ in abs EtOH $(96 \mathrm{~mL}$ ) as described for the preparation of 6 from 37. The crude hydrogenation product (off-white foamy solid) was chromatographed on silica gel column with AcOEt, as eluent, to afford the title compound $\mathbf{1 3}$ as a white 
foamy solid, which strongly binds the elution solvent. Removal of the entrapped solvent as in $\mathbf{6}$ gave $\mathbf{1 3}$ as a glass solid (665 mg, 94\%): ${ }^{1} \mathrm{H}$ NMR (600 MHz, DMSO- $\left.d_{6}\right) \delta 0.81\left(\mathrm{t}, 3 \mathrm{H}, J=7.1 \mathrm{~Hz}, \mathrm{CH}_{3}\left(\mathrm{CH}_{2}\right)_{3}\right), 0.99-1.13(\mathrm{~m}, 1 \mathrm{H}$, $\left.\mathrm{CH}_{3} \mathrm{CH}_{2} \mathrm{CHHCH}_{2}\right), 1.15-1.30\left(\mathrm{~m}, 3 \mathrm{H}, \mathrm{CH}_{3} \mathrm{CH}_{2} \mathrm{CHHCH}_{2}\right), 1.84-1.96\left(\mathrm{~m}, 1 \mathrm{H}, \mathrm{CH}_{3}\left(\mathrm{CH}_{2}\right)_{2} \mathrm{CHH}\right), 2.12-2.24(\mathrm{~m}, 1 \mathrm{H}$, $\left.\mathrm{CH}_{3}\left(\mathrm{CH}_{2}\right)_{2} \mathrm{CHH}\right), 2.37,2.38\left(\mathrm{~s}+\mathrm{s}, 3 \mathrm{H}, 4-\mathrm{CH}_{3}\right), 3.29-3.56$ (q, $\left.\mathrm{AB}, 2 \mathrm{H}, \mathrm{J}_{\mathrm{AB}}=18.0 \mathrm{~Hz}, 5-\mathrm{H}\right), 4.27\left(\mathrm{~s}, 1.45 \mathrm{H}, \mathrm{CH}_{2} \mathrm{CONHOH}\right.$, E-isomer), $4.57\left(\mathrm{~s}, 0.5 \mathrm{H}, \mathrm{CH}_{2} \mathrm{CONHOH}, Z\right.$-isomer), 7.30-7.45 (m, $5 \mathrm{H}$, aromatic $\left.\mathrm{H}\right), 8.92(\mathrm{~s}, 0.6 \mathrm{H}, \mathrm{CONHOH}, E-$ isomer), 9.34 (s, $0.2 \mathrm{H}, \mathrm{CONHOH}, \mathrm{Z}$-isomer), $10.24(\mathrm{~s}, 0.2 \mathrm{H}, \mathrm{CONHOH}, \mathrm{Z}$-isomer), 10.70 (s, 0.6H, CONHOH, Eisomer); ${ }^{13} \mathrm{C}$ NMR (50 MHz, DMSO-d $\left.d_{6}\right) \delta 13.9\left(\mathrm{CH}_{3}\left(\mathrm{CH}_{2}\right)_{3}\right), 22.4\left(\mathrm{CH}_{3} \mathrm{CH}_{2}\left(\mathrm{CH}_{2}\right)_{2}\right), 25.2\left(\mathrm{CH}_{3} \mathrm{CH}_{2} \mathrm{CH}_{2} \mathrm{CH}_{2}\right), 35.2$ $\left(\mathrm{CH}_{3}\left(\mathrm{CH}_{2}\right)_{2} \mathrm{CH}_{2}\right), 36.8\left(4-\mathrm{CH}_{3}\right), 39.0\left(\mathrm{CH}_{2} \mathrm{CONHOH}, \mathrm{E}\right.$-isomer), 39.8 ( $\mathrm{CH}_{2} \mathrm{CONHOH}, \mathrm{Z}$-isomer), 53.9 (5-C), 69.9 (3-C), 127.3, 128.0, 128.3 (2,3,4,5,6-aromatic C), 136.2 (1-aromatic C), 163.8 (CONHOH, E-isomer), 169.2 (CONHOH, Zisomer), 169.7, $173.3(2,6-\mathrm{C})$; HRMS (ESI): $[\mathrm{M}+\mathrm{H}]^{+}$calcd for $\mathrm{C}_{17} \mathrm{H}_{23} \mathrm{~N}_{3} \mathrm{O}_{4}, 334.1767$, found 334.1761. The hydrochloride salt $(\mathbf{1 3} \cdot \mathrm{HCl})$ was prepared as described for $12 \cdot \mathrm{HCl}$. Mp $162-166{ }^{\circ} \mathrm{C}$ (dec). Anal. Calcd for $\mathrm{C}_{17} \mathrm{H}_{24} \mathrm{ClN}_{3} \mathrm{O}_{4}$ : C, 55.21; $\mathrm{H}, 6.54 ; \mathrm{N}, 11.36$; Found: $\mathrm{C}, 55.52 ; \mathrm{H}, 6.23 ; \mathrm{N}, 11.56$.

\subsubsection{3-(4-Fluorophenyl)-N-hydroxy-3,4-dimethyl-2,6-dioxo-1-piperazineacetamide 14}

The $\mathrm{N}$-benzyloxy precursor $\mathbf{6 4}$ was prepared from carboxylic acid $\mathbf{6 0}(1 \mathrm{~g}, 3.4 \mathrm{mmol})$ in dry THF-DMF 4:1 following the procedure described for the preparation of compound $\mathbf{4 0}$ (precursor for $\mathbf{9}$ ). The crude oil was purified by column chromatography on silica gel eluting with AcOEt- $n$-hexane 1:2 and then 1:1 to afford 64 as a clear glass oil (1.03 g, 76\%). This compound appears in the ${ }^{1} \mathrm{H}$ and ${ }^{13} \mathrm{C}$ NMR spectra as a mixture of $E / Z$ conformers (not assigned). ${ }^{1} \mathrm{H} N M R\left(400 \mathrm{MHz}, \mathrm{CDCl}_{3}\right) \delta 1.63\left(\mathrm{~s}, 3 \mathrm{H}, 3-\mathrm{CH}_{3}\right), 2.53\left(\mathrm{~s}, 3 \mathrm{H}, 4-\mathrm{CH}_{3}\right), 3.37-3.66\left(\mathrm{q}, \mathrm{AB}, 2 \mathrm{H}, J_{A B}=18.0 \mathrm{~Hz}\right.$, 5-H), 4.26-4.50 (br d, 0.9H, J=21.2 Hz, $\mathrm{CH}_{2} \mathrm{CONHOCH}_{2} \mathrm{Ph}$ ), 4.69-4.79 (br s, $1 \mathrm{H}, \mathrm{CH}_{2} \mathrm{CONHOCH}_{2} \mathrm{Ph}$ ), 4.91 (s, $2 \mathrm{H}$, $\mathrm{CONHOCH}_{2} \mathrm{Ph}$ ), $7.04\left(\mathrm{t}, 2 \mathrm{H}, \mathrm{J} \simeq 8.7 \mathrm{~Hz}, 3,5-\mathrm{H}\right.$ for 4- $\mathrm{FC}_{6} \mathrm{H}_{4}$ ), 7.29-7.50 (complex m, 7H, $\mathrm{C}_{6} \mathrm{H}_{5}, 2,6-\mathrm{H}$ for 4- $\mathrm{FC}_{6} \mathrm{H}_{4}$ ), 8.31 (br s, 0.4H, CONHOCH $2 \mathrm{Ph}$ ), 8.73 (br s, $\left.0.4 \mathrm{H}, \mathrm{CONHOCH}_{2} \mathrm{Ph}\right) ;{ }^{13} \mathrm{C} \mathrm{NMR}\left(150 \mathrm{MHz}, \mathrm{CDCl}_{3}\right) \delta 23.9\left(3-\mathrm{CH}_{3}\right), 38.2$ (4$\left.\mathrm{CH}_{3}\right)$, 39.6 $\left(\mathrm{CH}_{2} \mathrm{CONHOCH}_{2} \mathrm{Ph}\right), 54.9(5-\mathrm{C}), 67.5(3-\mathrm{C}), 78.4,79.7\left(\mathrm{CONHOCH}_{2} \mathrm{Ph}\right), 115.7,115.9$ (d, $\mathrm{J}_{\mathrm{C}-\mathrm{F}}=21.2 \mathrm{~Hz}, 3,5-$ $\mathrm{C}$ for 4- $\left.\mathrm{FC}_{6} \mathrm{H}_{4}\right), 128.1,128.2$ (d, $\mathrm{J}_{C-\mathrm{F}}=7.5 \mathrm{~Hz}, 2,6-\mathrm{C}$ for $\left.4-\mathrm{FC}_{6} \mathrm{H}_{4}\right), 128.5,128.6,128.8,129.3,129.4(2,3,4,5,6-\mathrm{C}$ for $\left.\mathrm{C}_{6} \mathrm{H}_{5}\right), 134.3,135.2\left(1-\mathrm{C}\right.$ for $\left.\mathrm{C}_{6} \mathrm{H}_{5}\right), 136.6\left(1-\mathrm{C}\right.$ for $\left.4-\mathrm{FC}_{6} \mathrm{H}_{4}\right), 161.7,163.3\left(\mathrm{~d}, \mathrm{~J}_{\mathrm{C}-\mathrm{F}}=246 \mathrm{~Hz}, 4-\mathrm{C}\right.$ for $\left.4-\mathrm{FC}_{6} \mathrm{H}_{4}\right), 165.2$ (weak signal intensity), 170.3, $173.7\left(\mathrm{CONHOCH}_{2} \mathrm{Ph}, 2,6-\mathrm{C}\right)$; ESI ${ }^{+} \mathrm{MS}: \mathrm{m} / \mathrm{z} 400.2[\mathrm{M}+\mathrm{H}]^{+}$.

Compound 64 ( $1 \mathrm{~g}, 2.5 \mathrm{mmol}$ ) was subjected to catalytic hydrogenation $\left(\mathrm{H}_{2} / 10 \% \mathrm{Pd}-\mathrm{C}, 120 \mathrm{mg}\right)$ in abs EtOH (112 $\mathrm{mL}$ ) as described for the preparation of 6 from 37 . The crude hydrogenation product (off-white foamy solid) was chromatographed on silica gel column with AcOEt, as eluent, to afford the title compound $\mathbf{1 4}$ as a white foamy solid, which strongly binds the elution solvent. Removal of the entrapped solvent as in $\mathbf{6}$ gave $\mathbf{1 4}$ as a glass solid (703 mg, 91\%): ${ }^{1} \mathrm{H}$ NMR (400 MHz, DMSO-d 6 ) $\delta 1.56\left(\mathrm{~s}, 3 \mathrm{H}, 3-\mathrm{CH}_{3}\right), 2.35,2.37$ (s + s, 3H, 4- $\mathrm{CH}_{3}$ ), 3.33-3.60 (q, AB, $\left.2 \mathrm{H}, J_{A B} \simeq 18.0 \mathrm{~Hz}, 5-\mathrm{H}\right), 4.27$ (s, 1.4H, $\mathrm{CH}_{2} \mathrm{CONHOH}, E$-isomer), 4.57 (s, 0.5H, $\mathrm{CH}_{2} \mathrm{CONHOH}, Z$-isomer), 7.19 (t, $2 \mathrm{H}$, $J=8.7 \mathrm{~Hz}, 3,5$-aromatic $\mathrm{H}), 7.46-7.54(\sim \mathrm{q}, 2 \mathrm{H}, J=4.4,5.4 \mathrm{~Hz}, 2,6$-aromatic $\mathrm{H}), 8.96$ (s, 0.7H, CONHOH, E-isomer), 9.39 (s, 0.2H, CONHOH, Z-isomer), 10.28 (s, 0.2H, CONHOH, Z-isomer), 10.74 (s, 0.6H, CONHOH, E-isomer); ${ }^{13} \mathrm{C}$ $\operatorname{NMR}\left(50 \mathrm{MHz}, \mathrm{DMSO}-d_{6}\right) \delta 21.7,21.8\left(3-\mathrm{CH}_{3}\right), 37.2\left(4-\mathrm{CH}_{3}\right), 38.9\left(\mathrm{CH}_{2} \mathrm{CONHOH}, \mathrm{E}\right.$-isomer), $39.7\left(\mathrm{CH}_{2} \mathrm{CONHOH}, \mathrm{Z}-\right.$ isomer), $53.8(5-C), 66.8(3-C), 115.0,115.5$ (d, $J_{C-F}=21.1 \mathrm{~Hz}, 3,5$-aromatic C), 128.7, 128.9 (d, $J_{C-F}=7.9 \mathrm{~Hz}, 2,6-$ aromatic C), 136.9, 137.0 (d, $J_{C-F}=3.1 \mathrm{~Hz}, 1$-aromatic C), 159.2, 164.0 (d, $J_{C-F}=243 \mathrm{~Hz}$, 4-aromatic C), 163.8 (CONHOH, E-isomer), 169.2 (CONHOH, Z-isomer), 169.6, 173.4 (2,6-C); HRMS (ESI): [M+Na ${ }^{+}$calcd for $\mathrm{C}_{14} \mathrm{H}_{16} \mathrm{FN}_{3} \mathrm{O}_{4}, 332.1023$, found 332.1021. The hydrochloride salt $(\mathbf{1 4} \cdot \mathbf{H C l})$ was prepared as described for $\mathbf{1 2} \cdot \mathbf{H C l}$ (decomposed gradually above $126^{\circ} \mathrm{C}$ ). Anal. Calcd for $\mathrm{C}_{14} \mathrm{H}_{17} \mathrm{ClFN}_{3} \mathrm{O}_{4}: \mathrm{C}, 48.63 ; \mathrm{H}, 4.96 ; \mathrm{N}, 12.15$; Found: C, 48.34; H, 5.27; N, 11.94 .

\subsubsection{N-Hydroxy-3,4-dimethyl-3-(4-nitrophenyl)-2,6-dioxo-1-piperazineacetamide 15}

The $N$-(4-methoxybenzyloxy) precursor 65 was prepared from 4-methoxybenzyl ester 56 ( $1 \mathrm{~g}, 2.27 \mathrm{mmol})$ by the same method as for $\mathbf{4 1}$ (precursor for 10). The crude oil was purified by column chromatography on silica gel with AcOEt- $n$-hexane 1:1, as eluent, to afford 65 as a yellowish thick oil ( $445 \mathrm{mg}, 43 \%$ yield over two steps). This compound appears in the ${ }^{1} \mathrm{H}$ NMR spectrum as two pairs of rotamers for the $E / Z$ isomers as deduced both from the number of signals ad their integration. ${ }^{1} \mathrm{H} \mathrm{NMR}\left(400 \mathrm{MHz}, \mathrm{CDCl}_{3}\right) \delta[1.60(\mathrm{~s}), 1.66(\mathrm{~s})] 3 \mathrm{H}, 3-\mathrm{CH}_{3},[2.21(\mathrm{~s})$, 
$2.54(\mathrm{~s})] 3 \mathrm{H}, 4-\mathrm{CH}_{3},[2.82-3.03(\mathrm{q}, J=13.4,16.3 \mathrm{~Hz}$ ), 3.43-3.62 (q, AB, JAB $=18.1 \mathrm{~Hz})] 2 \mathrm{H}, 5-\mathrm{H},[3.77$ (s), $3.80(\mathrm{~s})]$ $\mathrm{OCH}_{3}$, 4.26-4.47 (q, J=14.0, $16.7 \mathrm{~Hz}, \mathrm{CH}_{2} \mathrm{CONHOCH}_{2} \mathrm{C}_{6} \mathrm{H}_{4} \mathrm{OCH}_{3}-4$ ), 4.60 (s, $\mathrm{CONHOCH}_{2} \mathrm{C}_{6} \mathrm{H}_{4} \mathrm{OCH}_{3}-4$ ), [4.69 (s), 4.71 (s)] $\mathrm{CH}_{2} \mathrm{CONHOCH}_{2} \mathrm{C}_{6} \mathrm{H}_{4} \mathrm{OCH}_{3}-4,4.83$ (s, $\left.\mathrm{CONHOCH}_{2} \mathrm{C}_{6} \mathrm{H}_{4} \mathrm{OCH}_{3}-4\right)$, [6.83 (d, J=8.3 Hz), 6.89 (d, J=8.1 Hz)] 3,5-H for 4- $\left.\mathrm{CH}_{3} \mathrm{OC}_{6} \mathrm{H}_{4}\right),\left[7.23\left(\mathrm{~d}, \mathrm{~J}=8.1 \mathrm{~Hz}\right.\right.$ ), 7.26-7.37 (q, J=8.4, $9.0 \mathrm{~Hz}$ )] 2,6- $\mathrm{H}$ for 4- $\left.\mathrm{CH}_{3} \mathrm{OC}_{6} \mathrm{H}_{4}\right),[7.63(\mathrm{~d}, J=7.6 \mathrm{~Hz}), 7.70(\mathrm{~d}$, $J=8.6 \mathrm{~Hz}$ )] 2,6- $\mathrm{H}$ for $\left.4-\mathrm{NO}_{2} \mathrm{C}_{6} \mathrm{H}_{4}\right),[8.05-8.17(\mathrm{~m}), 8.20(\mathrm{~d}, \mathrm{~J}=8.5 \mathrm{~Hz})] 3,5-\mathrm{H}$ for 4- $\left.\mathrm{NO}_{2} \mathrm{C}_{6} \mathrm{H}_{4}\right)$, [8.61 (br s), 9.09 (br s), 10.11 (br s), 10.38 (br s)] $\left.\mathrm{CONHOCH}_{2} \mathrm{C}_{6} \mathrm{H}_{4} \mathrm{OCH}_{3}-4\right) ;{ }^{13} \mathrm{C} \mathrm{NMR} \mathrm{(50} \mathrm{MHz,} \mathrm{CDCl} 3$ ) $\delta 23.3\left(3-\mathrm{CH}_{3}\right), 37.8$ (weak signal intensity), $38.2\left(4-\mathrm{CH}_{3}\right), 39.6\left(\mathrm{CH}_{2} \mathrm{CONHOCH}_{2} \mathrm{C}_{6} \mathrm{H}_{4} \mathrm{OCH}_{3}-4\right), 54.7$ (5-C), $55.3\left(\mathrm{OCH}_{3}\right), 67.8$ (3-C), 77.7, 79.4 ( $\left.\mathrm{CONHOCH}_{2} \mathrm{C}_{6} \mathrm{H}_{4} \mathrm{OCH}_{3}-4\right)$, 113.9, 114.1, 123.6, 124.1, 127.6, 128.2, 129.3, 130.2, 131.0, 131.1, 147.2, 147.7, 148.3 (aromatic C), 159.6, 160.1 ( $\left.\mathrm{CONHOCH}_{2} \mathrm{C}_{6} \mathrm{H}_{4} \mathrm{OCH}_{3}-4\right)$, 169.6, 172.7 (2,6-C); ESI+ MS: m/z 457.2 [M+H] .

Compound $65\left(460 \mathrm{mg}, 1.01 \mathrm{mmol}\right.$ ) was subjected to treatment with TFA $(7.4 \mathrm{~mL})$ and $\mathrm{Et}_{3} \mathrm{SiH}(742 \mu \mathrm{L})$ in dry $\mathrm{CH}_{2} \mathrm{Cl}_{2}(28 \mathrm{~mL}$ ) as described for the preparation of compound $\mathbf{1 0}$ from $\mathbf{4 1}$. The crude product (yellow foamy solid) was chromatographed on silica gel column eluting successively with AcOEt-n-hexane 2:1, AcOEt and AcOEt-MeOH 9:1 to afford the title compound $\mathbf{1 5}$ as a pale yellow foamy solid, which strongly binds the aforementioned solvents. Removal of the entrapped solvents as in $\mathbf{6}$ gave $\mathbf{1 5}$ as a light-yellow solid (237 $\mathrm{mg}$, 70\%): ${ }^{1} \mathrm{H}$ NMR (400 MHz, DMSO- $\left.d_{6}\right) \delta 1.61\left(\mathrm{~s}, 3 \mathrm{H}, 3-\mathrm{CH}_{3}\right), 2.36,2.39\left(\mathrm{~s}+\mathrm{s}, 3 \mathrm{H}, 4-\mathrm{CH}_{3}\right), 3.41-3.66(\mathrm{q}, \mathrm{AB}, 2 \mathrm{H}$, $J_{A B}=18.0 \mathrm{~Hz}, 5-\mathrm{H}$ ), $4.27\left(\mathrm{~s}, 1.4 \mathrm{H}, \mathrm{CH}_{2} \mathrm{CONHOH}, E\right.$-isomer), 4.51-4.65 (q, $\mathrm{AB}, 0.6 \mathrm{H}, \mathrm{J}_{A B}=17.0 \mathrm{~Hz}, \mathrm{CH}_{2} \mathrm{CONHOH}, \mathrm{Z}-$ isomer), $7.76(\mathrm{~d}, 2 \mathrm{H}, \mathrm{J}=8.8 \mathrm{~Hz}, 2,6$-aromatic $\mathrm{H}), 8.22(\mathrm{~d}, 2 \mathrm{H}, \mathrm{J}=8.8 \mathrm{~Hz}, 3,5$-aromatic $\mathrm{H}), 8.96(\mathrm{~s}, 0.7 \mathrm{H}, \mathrm{CONHOH}, E$ isomer), $9.40(\mathrm{~s}, 0.3 \mathrm{H}, \mathrm{CONHOH}, Z$-isomer), 10.30 (s, 0.2H, CONHOH, Z-isomer), 10.74 (s, 0.7H, CONHOH, Eisomer); ${ }^{13} \mathrm{C} \mathrm{NMR}\left(50 \mathrm{MHz}\right.$, DMSO-d $\left.d_{6}\right) \delta 20.9\left(3-\mathrm{CH}_{3}\right), 37.2\left(4-\mathrm{CH}_{3}\right), 38.9\left(\mathrm{CH}_{2} \mathrm{CONHOH}\right), 53.7(5-\mathrm{C}), 67.2(3-\mathrm{C})$, 123.6 (3,5-aromatic C), 128.2 (2,6-aromatic C), 147.1, 148.6 (1,4-aromatic C), 163.6 (CONHOH, $E$-isomer), 169.0 (CONHOH, Z-isomer), 169.3, 172.8 (2,6-C); HRMS (ESI): [M+H] $]^{+}$calcd for $\mathrm{C}_{14} \mathrm{H}_{16} \mathrm{~N}_{4} \mathrm{O}_{6}, 337.1148$, found 337.1157. The hydrochloride salt $(\mathbf{1 5} \cdot \mathbf{H C l})$ was prepared by the same way described for $\mathbf{1 0}$, and obtained as an off-white slightly hygroscopic solid (decomposed gradually above $166^{\circ} \mathrm{C}$ ). Anal. Calcd for $\mathrm{C}_{14} \mathrm{H}_{17} \mathrm{ClN}_{4} \mathrm{O}_{6}$ : $\mathrm{C}, 45.11 ; \mathrm{H}, 4.60$; N, 15.03; Found: C, 44.78; $H, 4.52 ; \mathrm{N}, 15.28$.

4.1.53. N-Hydroxy-3-methyl-2,6-dioxo-3-phenyl-4-propyl-1-piperazineacetamide Hydrochloride 16

The $\mathrm{N}$-benzyloxy precursor $\mathbf{7 4}$ was prepared from carboxylic acid $72(496 \mathrm{mg}, 1.63 \mathrm{mmol}$ ) following the procedure described for the preparation of compound $\mathbf{3 7}$ (precursor for 6). The crude yellowish thick oil was purified by column chromatography on silica gel with AcOEt- $n$-hexane 2:3, as eluent, to afford $\mathbf{7 4}$ as a glass solid (433 mg, 65\%). This compound appears in the ${ }^{1} \mathrm{H}$ and ${ }^{13} \mathrm{C}$ NMR spectra as a mixture of $E / Z$ conformers (not assigned). ${ }^{1} \mathrm{H}$ NMR $\left(400 \mathrm{MHz}, \mathrm{CDCl}_{3}\right) \delta 0.92\left(\mathrm{t}, 3 \mathrm{H}, \mathrm{J}=7.4 \mathrm{~Hz}, \mathrm{NCH}_{2} \mathrm{CH}_{2} \mathrm{CH}_{3}\right), 1.40-1.61\left(\mathrm{~m}, 2 \mathrm{H}, \mathrm{NCH}_{2} \mathrm{CH}_{2} \mathrm{CH}_{3}\right), 1.66$ (s, 3H, 3- $\left.\mathrm{CH}_{3}\right), 2.53-2.64$ (br s, $\left.1 \mathrm{H}, \mathrm{NCHHCH}_{2} \mathrm{CH}_{3}\right), 2.66-2.78\left(\mathrm{~m}, 1 \mathrm{H}, \mathrm{NCHHCH}_{2} \mathrm{CH}_{3}\right), 3.45-3.70$ (q, $\mathrm{AB}, 2 \mathrm{H}, \mathrm{J}_{\mathrm{AB}}=18.4$ $\mathrm{Hz}, 5-\mathrm{H}$ ), 4.21-4.55 ( $\mathrm{v}$ br d, 0.9H, $\mathrm{CH}_{2} \mathrm{CONHOCH}_{2} \mathrm{Ph}$ ), 4.66-4.82 (br s, 0.9H, $\mathrm{CH}_{2} \mathrm{CONHOCH}_{2} \mathrm{Ph}$ ), 4.91 (s, $2 \mathrm{H}$, $\mathrm{CONHOCH}_{2} \mathrm{Ph}$ ), 7.22-7.53 (complex m, 10H, aromatic $\mathrm{H}$ ), 7.99-8.40 ( $\mathrm{v}$ br s, 0.4H, $\mathrm{CONHOCH}_{2} \mathrm{Ph}$ ), 8.42-8.85 ( $\mathrm{v}$ br s, 0.3H, CONHOCH $2 \mathrm{Ph}) ;{ }^{13} \mathrm{C} \mathrm{NMR}\left(150 \mathrm{MHz}, \mathrm{CDCl}_{3}\right) \delta 11.7\left(\mathrm{NCH}_{2} \mathrm{CH}_{2} \mathrm{CH}_{3}\right), 21.4\left(\mathrm{NCH}_{2} \mathrm{CH}_{2} \mathrm{CH}_{3}\right), 23.3\left(3-\mathrm{CH}_{3}\right), 39.8$ $\left(\mathrm{CH}_{2} \mathrm{CONHOCH}_{2} \mathrm{Ph}\right), 50.7\left(\mathrm{NCH}_{2} \mathrm{CH}_{2} \mathrm{CH}_{3}\right), 51.1$ (5-C), 68.2 (3-C), 78.4, 79.7 ( $\left.\mathrm{CONHOCH}_{2} \mathrm{Ph}\right), 126.4,128.3,128.9$, 129.4, 134.3, 135.3, 141.4 (aromatic C), 165.1, $170.3\left(\mathrm{CONHOCH}_{2} \mathrm{Ph}\right), 170.7,174.0$ (2,6-C); ESI ${ }^{+} \mathrm{MS}: \mathrm{m} / \mathrm{z} 410.4$ $[\mathrm{M}+\mathrm{H}]^{+}$.

Compound 74 ( $390 \mathrm{mg}, 0.95 \mathrm{mmol}$ ) was subjected to catalytic hydrogenation $\left(\mathrm{H}_{2} / 10 \% \mathrm{Pd}-\mathrm{C}, 47 \mathrm{mg}\right.$ ), in abs EtOH $(43 \mathrm{~mL})$ as described for the preparation of 6 from 37. The crude hydrogenation material (off-white foamy solid) was chromatographed on silica gel column eluting first with AcOEt- $n$-hexane 2:1 and then AcOEt to afford the free base 16 as a white foamy solid, which strongly binds the elution solvents. (EI MS: m/z 319.1 ([M] $\left.{ }^{+}, 13\right), 291.1$ (14), 276.1 ([M- $\left.\left.\mathrm{CH}_{2} \mathrm{CH}_{2} \mathrm{CH}_{3}\right]^{+}, 11\right), 231.1$ (26), 174.0 (100)). This free base product was converted into the $\mathrm{HCl}$ salt as described for compound 12 to give $238 \mathrm{mg}(70 \%)$ of the title compound 16 as a white slightly hygroscopic solid (decomposed gradually above $115^{\circ} \mathrm{C}$ ); ${ }^{1} \mathrm{H}$ NMR $\left(400 \mathrm{MHz}\right.$, DMSO-d 6 ) $\delta 0.78\left(\mathrm{t}, 3 \mathrm{H}, J=7.2 \mathrm{~Hz},{ }^{+} \mathrm{NH}\left(\mathrm{CH}_{2}\right)_{2} \mathrm{CH}_{3}\right)$, 1.32-1.59 (complex m, $2 \mathrm{H},{ }^{+} \mathrm{NHCH}_{2} \mathrm{CH}_{2} \mathrm{CH}_{3}$ ), $1.73\left(\mathrm{~s}, 3 \mathrm{H}, 3-\mathrm{CH}_{3}\right), 2.34-2.53\left(\mathrm{~m}, 1 \mathrm{H},{ }^{+} \mathrm{NHCHHCH}_{2} \mathrm{CH}_{3}\right), 2.62-2.88(\mathrm{br}$ $\left.\mathrm{s}, 1 \mathrm{H},{ }^{+} \mathrm{NHCHHCH}_{2} \mathrm{CH}_{3}\right), 3.46-3.93\left(\mathrm{q}, \mathrm{AB}, 2 \mathrm{H}, \mathrm{J}_{\mathrm{AB}} \simeq 16.0 \mathrm{~Hz}, 5-\mathrm{H}\right), 4.28\left(\mathrm{~s}, 1.5 \mathrm{H}, \mathrm{CH}_{2} \mathrm{CONHOH}, E\right.$-isomer), 4.56 (s, 
0.5H, $\mathrm{CH}_{2} \mathrm{CONHOH}, \mathrm{Z}$-isomer), 6.52-8.41 (br m, 7H, $\mathrm{CONHOH},{ }^{+} \mathrm{NH}\left(\mathrm{CH}_{2}\right)_{2} \mathrm{CH}_{3}$, aromatic $\left.\mathrm{H}\right), 10.29$ (s, 0.2H,

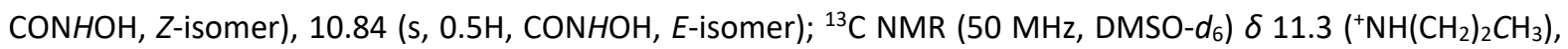
$19.8\left({ }^{+} \mathrm{NHCH}_{2} \mathrm{CH}_{2} \mathrm{CH}_{3}\right), 20.6\left(3-\mathrm{CH}_{3}\right), 39.5\left(\mathrm{CH}_{2} \mathrm{CONHOH}, \mathrm{E}\right.$-isomer), $40.4\left(\mathrm{CH}_{2} \mathrm{CONHOH}, \mathrm{Z}\right.$-isomer), 50.0 (5-C), 50.6 ( ${ }^{+} \mathrm{NHCH}_{2} \mathrm{CH}_{2} \mathrm{CH}_{3}$ ), 68.0 (3-C), 127.3, 128.6 (2,3,4,5,6-aromatic C), 139.1 (1-aromatic C), 163.5 (CONHOH, Eisomer), 168.9 (CONHOH, Z-isomer), 168.4, 172.6 (2,6-C). Anal. Calcd for $\mathrm{C}_{16} \mathrm{H}_{22} \mathrm{ClN}_{3} \mathrm{O}_{4}: \mathrm{C}, 54.01 ; \mathrm{H}, 6.23 ; \mathrm{N}$, 11.81; Found: C, 53.66; H, 6.62; N, 12.14.

Note. The above prepared free base 16 was partially decomposed on attempted drying at $62-64{ }^{\circ} \mathrm{C}$ under high vacuum $\left(10^{-2} \mathrm{mmHg}\right)$.

4.1.54. 4-Butyl-N-hydroxy-3-methyl-2,6-dioxo-3-phenyl-1-piperazineacetamide Hydrochloride 17

The $\mathrm{N}$-benzyloxy precursor 75 was prepared from carboxylic acid $73(465 \mathrm{mg}, 1.46 \mathrm{mmol}$ ) following the procedure described for the preparation of compound $\mathbf{3 7}$ (precursor for 6). The crude yellowish thick oil was purified by column chromatography on silica gel with AcOEt-n-hexane 1:2, as eluent, to afford 75 as a glass oil, which solidified under cooling (white solid, $400 \mathrm{mg}, 65 \%$ yield): $\mathrm{mp} 128-129^{\circ} \mathrm{C}$ (AcOEt/Et $2 \mathrm{O}$ 1:10- $n$-pentane). This compound appears in the ${ }^{1} \mathrm{H}$ and ${ }^{13} \mathrm{C} N M R$ spectra as a mixture of $E / Z$ conformers (not assigned). ${ }^{1} \mathrm{H} N M R$ $\left(400 \mathrm{MHz}, \mathrm{CDCl}_{3}\right) \delta 0.90\left(\mathrm{t}, 3 \mathrm{H}, \mathrm{J}=7.3 \mathrm{~Hz}, \mathrm{~N}\left(\mathrm{CH}_{2}\right)_{3} \mathrm{CH}_{3}\right.$ ), 1.24-1.55 (complex m, 4H, NCH $\left.\mathrm{CH}_{2} \mathrm{CH}_{2} \mathrm{CH}_{3}\right), 1.49$ (s, 3H, 3- $\mathrm{CH}_{3}$ ), 2.50-2.67 (br s, $\left.1 \mathrm{H}, \mathrm{NCHH}\left(\mathrm{CH}_{2}\right)_{2} \mathrm{CH}_{3}\right), 2.69-2.82\left(\mathrm{~m}, 1 \mathrm{H}, \mathrm{NCHH}\left(\mathrm{CH}_{2}\right)_{2} \mathrm{CH}_{3}\right), 3.49-3.65$ (q, $\mathrm{AB}, 2 \mathrm{H}, \mathrm{J}_{\mathrm{AB}}=18.2$ $\mathrm{Hz}, 5-\mathrm{H})$, 4.15-4.53 ( $\mathrm{vbr}$ d, 0.8H, $\mathrm{CH}_{2} \mathrm{CONHOCH}_{2} \mathrm{Ph}$ ), 4.72 (s, 0.9H, $\left.\mathrm{CH}_{2} \mathrm{CONHOCH}_{2} \mathrm{Ph}\right), 4.89\left(\mathrm{~s}, 2 \mathrm{H}, \mathrm{CONHOCH}_{2} \mathrm{Ph}\right.$ ), 7.20-7.53 (complex $\mathrm{m}, 10 \mathrm{H}$, aromatic $\mathrm{H}$ ), 8.30-8.47 (br s, 0.4H, $\mathrm{CONHOCH}_{2} \mathrm{Ph}$ ), 8.67-8.91 (br s, $0.4 \mathrm{H}$,

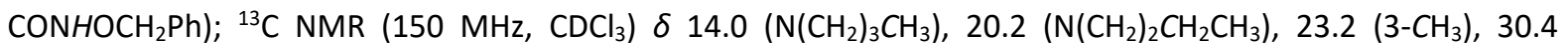
$\left(\mathrm{NCH}_{2} \mathrm{CH}_{2} \mathrm{CH}_{2} \mathrm{CH}_{3}\right), 39.7\left(\mathrm{CH}_{2} \mathrm{CONHOCH}_{2} \mathrm{Ph}\right), 48.4\left(\mathrm{NCH}_{2}\left(\mathrm{CH}_{2}\right)_{2} \mathrm{CH}_{3}\right), 51.0$ (5-C), 68.1 (3-C), 78.3, 79.7 ( $\mathrm{CONHOCH}_{2} \mathrm{Ph}$ ), 126.3, 128.1, 128.9, 129.4, 134.4, 135.3, 141.5 (aromatic C), 165.2, 170.3 (CONHOCH$\left.{ }_{2} \mathrm{Ph}\right)$ 170.9, 174.1 (2,6-C). Anal. Calcd for $\mathrm{C}_{24} \mathrm{H}_{29} \mathrm{~N}_{3} \mathrm{O}_{4}$ : C, 68.06; H, 6.90; N, 9.92; Found: C, 68.32; H, 7.02; N, 9.63.

Compound 75 ( $620 \mathrm{mg}, 1.46 \mathrm{mmol}$ ) was subjected to catalytic hydrogenation $\left(\mathrm{H}_{2} / 10 \% \mathrm{Pd}-\mathrm{C}, 74 \mathrm{mg}\right)$ in abs EtOH $(66 \mathrm{~mL})$ as described for the preparation of 6 from 37. The crude hydrogenation material (off-white foamy solid) was chromatographed on silica gel column with AcOEt- $n$-hexane 1:1, as eluent, to afford the free base $\mathbf{1 7}$ as a white foamy solid, which strongly binds the elution solvents. $\left(\mathrm{Cl}^{+} \mathrm{MS}: \mathrm{m} / \mathrm{z} 334.1\left([\mathrm{M}+\mathrm{H}]^{+}, 7\right), 333.1\left([\mathrm{M}]^{+}, 6\right)\right.$, 305.1 (17), 276.0 ([M- $\left.\left.\mathrm{CH}_{2} \mathrm{CH}_{2} \mathrm{CH}_{2} \mathrm{CH}_{3}\right]^{+}, 10\right), 245.1$ (35), 188.1 (100)). This free base product was converted into the $\mathrm{HCl}$ salt as described for compound 12 to give $369 \mathrm{mg}(68 \%)$ of the title compound $\mathbf{1 7}$ as a white slightly hygroscopic solid (decomposed gradually above $108{ }^{\circ} \mathrm{C}$ ); ${ }^{1} \mathrm{H}$ NMR $\left(400 \mathrm{MHz}\right.$, DMSO- $\left.d_{6}\right) \delta 0.67(\mathrm{t}, 3 \mathrm{H}, \mathrm{J}=7.4 \mathrm{~Hz}$, ${ }^{+} \mathrm{NH}\left(\mathrm{CH}_{2}\right)_{3} \mathrm{CH}_{3}$ ), 1.0-1.20 (complex m, 2H, ${ }^{+} \mathrm{NH}\left(\mathrm{CH}_{2}\right)_{2} \mathrm{CH}_{2} \mathrm{CH}_{3}$ ), 1.22-1.36 (complex m, 2H, ${ }^{+} \mathrm{NHCH}_{2} \mathrm{CH}_{2} \mathrm{CH}_{2} \mathrm{CH}_{3}$ ), 1.54 (s, 3H, 3- $\left.\mathrm{CH}_{3}\right), 2.31-2.44\left(\mathrm{~m}, 2 \mathrm{H},{ }^{+} \mathrm{NHCH}_{2}\left(\mathrm{CH}_{2}\right)_{2} \mathrm{CH}_{3}\right), 3.30-3.68$ (q, $\left.\mathrm{AB}, 2 \mathrm{H}, J_{A B}=17.4 \mathrm{~Hz}, 5-\mathrm{H}\right), 4.14$ (s, $1.5 \mathrm{H}$, $\mathrm{CH}_{2} \mathrm{CONHOH}, \mathrm{E}$-isomer), 4.43 (s, 0.5H, $\mathrm{CH}_{2} \mathrm{CONHOH}, \mathrm{Z}$-isomer), 5.40-6.80 ( $\mathrm{v}$ br s, $2 \mathrm{H}, \mathrm{CONHOH},{ }^{+} \mathrm{NH}\left(\mathrm{CH}_{2}\right)_{3} \mathrm{CH}_{3}$ ), 7.15-7.48 (m, $5 \mathrm{H}$, aromatic $\mathrm{H}), 10.14\left(\mathrm{~s}, 0.2 \mathrm{H}, \mathrm{CONHOH}, Z\right.$-isomer), 10.66 (s, 0.5H, CONHOH, $E$-isomer); ${ }^{13} \mathrm{C} \mathrm{NMR}$ $\left(50 \mathrm{MHz}, \mathrm{DMSO}-d_{6}\right) \delta 13.6\left({ }^{+} \mathrm{NH}\left(\mathrm{CH}_{2}\right)_{3} \mathrm{CH}_{3}\right), 19.5\left({ }^{+} \mathrm{NH}\left(\mathrm{CH}_{2}\right)_{2} \mathrm{CH}_{2} \mathrm{CH}_{3}\right), 20.7\left(3-\mathrm{CH}_{3}\right), 29.0\left({ }^{+} \mathrm{NHCH}_{2} \mathrm{CH}_{2} \mathrm{CH}_{2} \mathrm{CH}_{3}\right), 39.1$ ( $\mathrm{CH}_{2} \mathrm{CONHOH}, \mathrm{E}$-isomer), $39.9\left(\mathrm{CH}_{2} \mathrm{CONHOH}, \mathrm{Z}\right.$-isomer), 48.0 ( $\left.{ }^{+} \mathrm{NHCH}_{2}\left(\mathrm{CH}_{2}\right)_{2} \mathrm{CH}_{3}\right), 50.1$ (5-C), 67.7 (3-C), 126.9, 128.1, 128.4 (2,3,4,5,6-aromatic C), 140.4 (1-aromatic C), 163.6 (CONHOH, E-isomer), 169.0 (CONHOH, Zisomer), 169.1, $173.2(2,6-\mathrm{C})$. Anal. Calcd for $\mathrm{C}_{17} \mathrm{H}_{24} \mathrm{ClN}_{3} \mathrm{O}_{4}: \mathrm{C}, 55.20 ; \mathrm{H}, 6.54 ; \mathrm{N}, 11.36$; Found: $\mathrm{C}, 54.83 ; \mathrm{H}, 6.86$; $\mathrm{N}, 11.02$.

Note. The above prepared free base 17 was partially decomposed on attempted drying at $62-64{ }^{\circ} \mathrm{C}$ under high vacuum $\left(10^{-2} \mathrm{mmHg}\right)$.

\subsection{Biological Assays}

\subsubsection{Trypanocidal assays}

Bloodstream form $T$. brucei (strain 221) were cultured in modified Iscove's medium, as outlined previously [11]. Eight-point potency curves were performed in 96 well plates (200 $\mu \mathrm{L}$ volumes), and the compound concentrations that inhibited growth by $50 \%$ (IC50) and $90 \%$ (IC90) were determined. Parasites were first diluted to $2.5 \times 10^{4} \mathrm{~mL}^{-1}$, compounds were added at range of concentrations, and the plates incubated at $37^{\circ} \mathrm{C}$. 
Resazurin was added after $48 \mathrm{~h}$, and the plates incubated for a further $16 \mathrm{~h}$. Fluorescence intensities were determined using a BMG FLUOstar Omega (excitation $545 \mathrm{~nm}$, emission $590 \mathrm{~nm}$ ). Data were analysed using Graph Pad Prism 7 software. Values are expressed as IC50 \pm SD and are the average of three independent replicates.

\subsubsection{In vitro cytotoxicity assays on rat skeletal myoblast $L 6$ cells}

Cytotoxicity against $L 6$ cells was assessed using microtitre plates. Briefly, cells were seeded in triplicate at $1 \times$ $10^{4} \mathrm{~mL}^{-1}$ in growth medium containing different compound concentrations. The plates were incubated for 6 days at $37{ }^{\circ} \mathrm{C}$ and resazurin then added to each well. After a further $8 \mathrm{~h}$ incubation, the fluorescence was determined using a Spectramax plate reader.

\section{References}

1. Trypanosomiasis, human African (sleeping sickness); 16 February 2018; World Health Organization: Geneva, 2018.

2. Baker $\mathrm{CH}$, Welburn SC. The long wait for a new drug for Human African Trypanosomiasis. Trends Parasitol. 34(10), 818-827 (2018).

3. Ruiz-Postigo JA, Franco JR, Lado M. Human African trypanosomiasis in South Sudan: how can we prevent a new epidemic? PLoS Negl. Trop. Dis. 6, e1541 (2012).

4. Büscher P, Cecchi G, Jamonneau V, Priotto G, Human African trypanosomiasis. Lancet 390, 2397-2409 (2017).

- An excellent review that describes the epidemiology, clinical features, diagnosis and treatment of Human African trypanosomiasis.

5. Fytas C, Zoidis G, Tzoutzas N, Taylor MC, Fytas G, Kelly JM. Novel lipophilic acetohydroxamic acid derivatives based on conformationally constrained spiro carbocyclic 2,6-diketopiperazine scaffolds with potent trypanocidal activity. J. Med. Chem. 54 (14), 5250-5254 (2011).

- An important investigation on the development of a novel antitrypanocidal agents.

6. Zoidis G, Tsotinis A, Tsatsaroni A, Taylor MC, Kelly JM, Efstathiou A, Smirlis D, Fytas G. Lipophilic conformationally constrained spiro carbocyclic 2,6-diketopiperazine-1-acetohydroxamic acid analogues as trypanocidal and leishmanicidal agents: An extended SAR study. Chem. Biol. Drug Des. 91(2), 408-421 (2018).

7. Fytas C, Zoidis G, Fytas G. A facile and effective synthesis of lipophilic 2,6-diketopiperazine analogues. Tetrahedron 64, 6749-6754 (2008).

8. Tsatsaroni A, Zoidis G, Zoumpoulakis P, Tsotinis T, Taylor MC, Kelly JM, Fytas G. An E/Z conformational behaviour study on the trypanocidal action of lipophilic spiro carbocyclic 2,6-diketopiperazine-1acetohydroxamic acids. Tet. Lett. 54 (25), 3238-3240 (2013).

9. Malik G, Guinchard X, Crich D. Asymmetric synthesis of polyhydroxylated $N$-alkoxypiperidines by ringclosing double reductive amination: Facile preparation of Isofagomine and analogues. Org. Lett. 14(2), 596-599 (2012).

10. Safir SR. Method for hypnosis and tranquilization with piperazinodione derivatives, US Patent (1965) Patent no: US3194731.

11. Taylor MC, McLatchie A, Kelly JM. Evidence that transport of iron from the lysosome to the cytosol in African trypanosomes is mediated by a mucolipin orthologue. Mol Microbiol. 89 (3), 420-432 (2013). 